\title{
Rational Design and Synthesis of Selective PRMT4 Inhibitors: a New Chemotype for Development of Cancer Therapeutics
}

\author{
Mathew Sutherland ${ }^{\mathrm{a}}$, Alice Li ${ }^{\mathrm{b}}$, Anissa Kaghad ${ }^{\mathrm{a}}$, Dimitrios Panagopoulos ${ }^{\mathrm{a}}$, Fengling Li ${ }^{\mathrm{b}}$, Magda- \\ lena Szewczyk ${ }^{\mathrm{b}}$, David Smil ${ }^{\mathrm{b}, \mathrm{c}}$, Cora Scholten ${ }^{\mathrm{d}}$, Léa Bouchéd ${ }^{\text {, Timo Stellfeld }}{ }^{\mathrm{d}, \mathrm{e}}$, Cheryl H. Ar- \\ rowsmith $^{\text {b,f }}$, Dalia Barsyte ${ }^{\mathrm{b}}$, Masoud Vedadi,g, Ingo V. Hartung ${ }^{\mathrm{d}, \mathrm{h}}$, Holger Steuber ${ }^{\mathrm{d}, \mathrm{e}}$, Robert Brit- \\ ton $^{\text {a* }}$, and Vijayaratnam Santhakumar ${ }^{\mathrm{b} *}$ \\ ${ }^{a}$ Department of Chemistry, Simon Fraser University, 8888 University Drive, Burnaby, BC, Canada \\ bStructural Genomics Consortium, MaRS Centre, South Tower, 101 College Street, Suite 70o, University of Toronto, Toronto, ON, \\ Canada \\ cCurrent address: Ontario Institute for Cancer Research, 661 University Ave, Toronto, ON M5G 1M1 \\ dBayer A.G., Research and Development, Pharmaceuticals, Open Innovation, 13442 Berlin, Germany \\ eCurrent address: Innovation Campus Berlin - Nuvisan ICB GmbH, Muellerstrasse 178, 13353 Berlin, Germany \\ fPrincess Margaret Cancer Centre and Department of Medical Biophysics, University of Toronto, Toronto, ON, M5G 2M9, Can- \\ ada \\ gDepartment of Pharmacology and Toxicology, University of Toronto Toronto, ON, Canada \\ hCurrent address: Merck Healthcare KGaA, Frankfurter Straße 250 | Postcode: D50/902 | 64293 Darmstadt | Germany
}

\begin{abstract}
Protein arginine N-methyl transferase 4 (PRMT4) asymmetrically dimethylates arginine residues of histone $\mathrm{H}_{3}$ and non-histone proteins. The overexpression of PRMT 4 in several cancers has stimulated interest in the discovery of inhibitors as biological tools and potentially therapeutics. While several PRMT4 inhibitors have been reported, most display poor selectivity against other members of the PRMT family of methyl transferases. Here, we report the structure-based design of a new class of alanine containing 3-arylindoles as potent and selective PRMT4 inhibitors and describe key structure activity relationships for this class of compounds.
\end{abstract}

Key words: PRMT4, PRMT6, Arginine methyl transferase, BAF155 Methylation, PRMT6 co-crystal structure, structure-based drug design 
bioRxiv preprint doi: https://doi.org/10.1101/2020.11.17.387233; this version posted November 18, 2020. The copyright holder for this preprint (which was not certified by peer review) is the author/funder, who has granted bioRxiv a license to display the preprint in perpetuity. It is made available under aCC-BY-NC-ND 4.0 International license.

Arginine methyl transferases catalyze both symmetric and asymmetric methylation of arginine residues in the histone $\mathrm{H}_{3}$ proteins using the methyl group from S-adenosyl-L-methionine (SAM). ${ }^{1}$ These methyl transferases regulate a variety of biological processes including transcriptional activation, ${ }^{2}$ RNA splicing, ${ }^{3}$ cell cycle regulation, ${ }^{4}$ DNA damage response, 5 and cell differentiation, ${ }^{6}$ while also catalyzing the methylation of a variety of non-histone proteins. 7 Type I arginine methyl transferases (PRMTs), PRMT1, 2, 3, 4, 6, and 8, catalyze mono and asymmetric dimethylation. The Type II PRMTs PRMT5 and 9 catalyze mono and symmetric di-methylation while $\mathrm{PRMT}_{7}$ catalyzes mono methylation of arginines. ${ }^{8}$

$\mathrm{PRMT}_{4}$ has been implicated in several malignancies and is highly overexpressed in $\sim 75 \%$ of colorectal cancers 9 as well as in prostate carcinoma and androgen-independent prostate carcinoma. ${ }^{10} \mathrm{PRMT}_{4}$ is a critical factor in the pathway of estrogen-stimulated breast cancer growth ${ }^{11}$ and its overexpression is associated with poor prognosis in this disease..$^{12}$ Knockout studies in breast cancer cell lines show that PRMT4 regulates breast cancer cell migration and metastasis..$^{13}$ Moreover, pharmacological inhibition of $\mathrm{PRMT}_{4}$ with selective inhibitors is effective in reducing the growth of multiple myeloma cell lines ${ }^{14}$ as well as in vivo mouse models of multiple myeloma ${ }^{15}$.

The majority of reported PRMT4 inhibitors shows moderate to poor selectivity against other Type I PRMTs ${ }^{16,17}$ and/or lack of cellular activity, ${ }^{18-20}$ with the notable exceptions of PRMT4 selective chemical probes 1 and 2 reported by Structural Genomics Consortium ${ }^{14,21}$ and Epizyme, ${ }^{15}$ respectively. Here, we report the development of indole based, potent and PRMT4 selective inhibitors starting from a dual PRMT4/6 inhibitor scaffold. Notably, we relied on the co-crystal structure of a hit compound za (Figure 1) with PRMT6 and molecular modeling with reported $\mathrm{PRMT}_{4}$ structures to design $\mathrm{PRMT}_{4}$ selective inhibitors and identify key structural features relevant to PRMT4 selectivity.

A

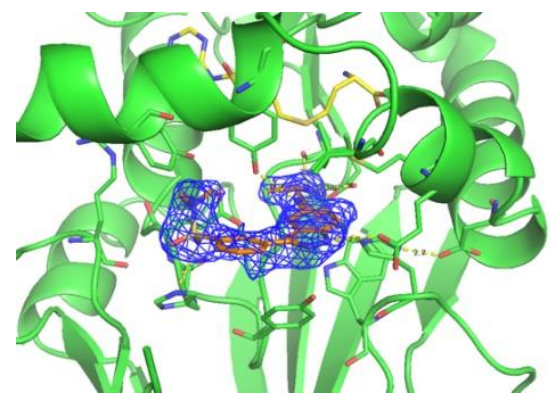

B

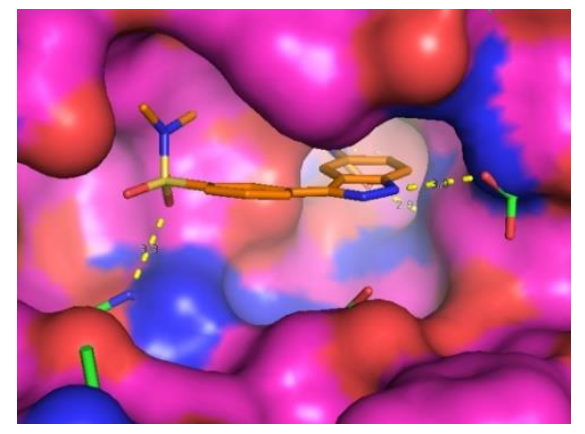

Figure 2. (A) The PRMT6-bound structure of 3a; (B) PRMT6bound conformation of za superimposed in binding site of
$\mathrm{PRMT}_{4}$ highlights a hydrophobic pocket (occupied by sulfonamide group) not present in PRMT6.

Owing to its potent PRMT4 inhibitory activity $\left(\mathrm{IC}_{50} 0.06 \mu \mathrm{M}\right)$ and moderate selectivity over PRMT6 family (8-fold), the indazole za (entry 1 , Table 1 ) was selected as a starting point for the development of a selective PRMT4 inhibitor. Unfortunately, attempts to co-crystallize 3 a or the structurally related indazole 4a (Figure 1) with $\mathrm{PRMT}_{4}$ were unsuccessful. However, we were able to obtain the co-crystal structure of za with PRMT6 (Figure 2A). Based on this structural insight, superimposition of the PRMT6-bound structure of indazole $3 a$ in the reported $\mathrm{PRMT}_{4}$ structure ${ }^{19}$ (Figure $2 \mathrm{~B}$ ) suggested that the corresponding indole may improve selectivity for PRMT4. Specifically, in the PRMT6-bound structure of $\mathbf{3 a}$, H-bonding between Glu59 and the indazole $\mathrm{NH}$ was identified as a key binding interaction. Thus, it was proposed that decreasing the acidity of the N-H from indazole $(\mathrm{pKa} \sim 14)$ to indole $(\mathrm{pKa} \sim 21)$ would attenuate interactions with PRMT6. Additionally, the reduced polar surface area (PSA) of the corresponding indole would expectedly result in improved hydrophobic interactions with $\mathrm{PRMT}_{4}$ (Figure $2 \mathrm{~B}$ ). To test this hypothesis, indole 4b was synthesized following the synthetic sequence de-

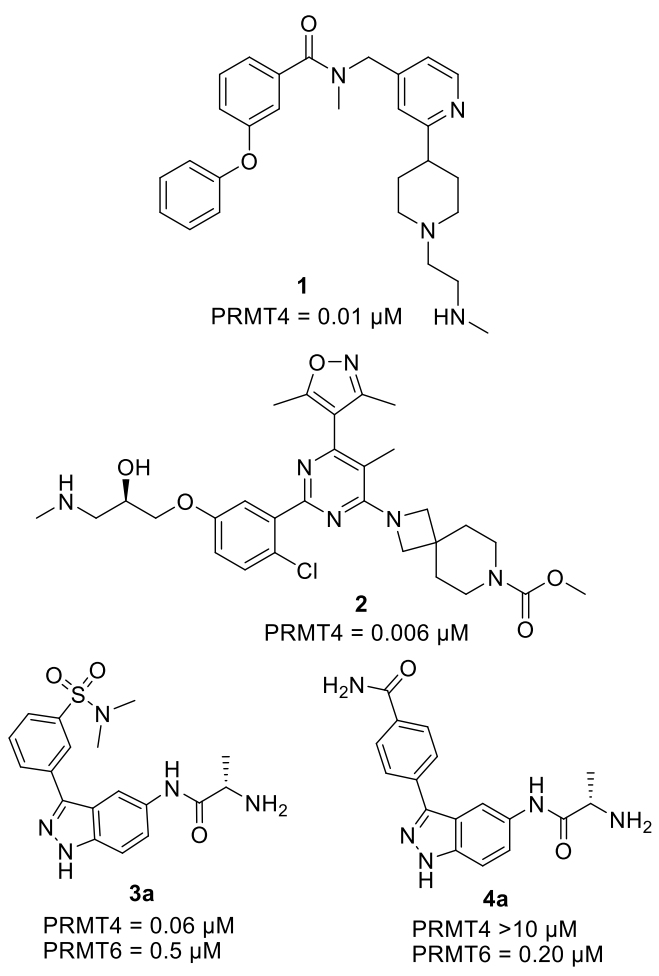

Figure 1. PRMT 4 inhibitors 1 and 2 reported by the Structural Genomics Consortium and Epizyme, respectively, and dual PRMT4/6 inhibitors identified through screening campaigns

scribed in Scheme 1. PRMT4 and PRMT6 activity assays were preformed according to the published procedures ${ }^{14}$. and we were pleased to find that this compound showed a moderate 
bioRxiv preprint doi: https://doi.org/10.1101/2020.11.17.387233; this version posted November $18,2020$. The copyright holder for this preprint (which was not certified by peer review) is the author/funder, who has granted bioRxiv a license to display the preprint in perpetuity. It is made available under aCC-BY-NC-ND 4.0 International license.

loss in PRMT6 activity and coincident gain in PRMT4 activity compared to $4 \mathbf{a}$ (Table 1 , entry 2 and 3 ). From a small collection of indazoles, it was noted that replacement of the amide with a meta-sulfonamide (entries 1,2 ) resulted in improved $\mathrm{PRMT}_{4}$ activity. Inspired by these observations, the corresponding meta-methyl sulfone analogue of indole $\mathbf{4} \mathbf{b}$ was prepared (entry 4), resulting in further improved PRMT 4 activity and selectivity. Notably, the meta-methyl sulfone $\mathbf{3 b}$ (entry 4 ) proved to be 197 -fold selective against PRMT6 $\left(\mathrm{PRMT}_{4} \mathrm{IC}_{50}=\right.$ $40 \mathrm{nM}$ ) and >50-fold selective against PRMT1,3,5-9. Docking studies of the sulfonamide $3 a$ in both PRMT4 and PRMT6 suggested that the improved PRMT4 activity is likely due to interactions within the unique hydrophobic binding pocket in $\mathrm{PRMT}_{4}$ (Figure $2 \mathrm{~B}$ ). This additional subpocket in $\mathrm{PRMT}_{4}$ is mainly created by Gln 149 and Phe 153, while the corresponding less space-demanding residues Leu 46 and Cys 50 do not generate s similar pocket in PRMT6.

Table 1: PRMT4 and PRMT6 activity of indazoles and indoles 3 and 4 .
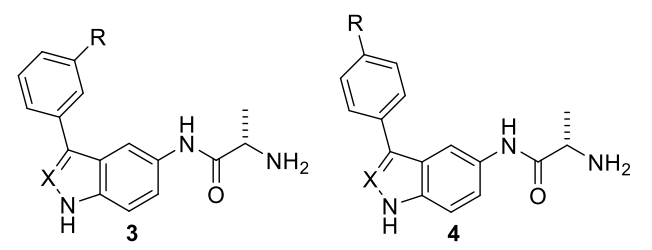

$\begin{array}{ccccccc}\text { entry } & \text { comp. } & \mathrm{X} & \mathrm{R} & \begin{array}{c}\mathrm{PRMT}_{4} \\ \left(\mathrm{IC}_{50}\right. \\ \mu \mathrm{M})\end{array} & \begin{array}{c}\mathrm{PRMT6} \\ \left(\mathrm{IC}_{50}\right. \\ \mu \mathrm{M})\end{array} & \begin{array}{c}\mathrm{PRMT}_{4} \\ \text { selec- } \\ \text { tivity }\end{array} \\ 1 & 3 \mathrm{a} & \mathrm{N} & \begin{array}{c}\mathrm{SO}_{2} \mathrm{NMe} \\ 2\end{array} & 0.06 & 0.5 & 8.3 \\ 2 & 4 \mathrm{a} & \mathrm{N} & \mathrm{CONH}_{2} & >10 & 0.2 & <0.02 \\ 3 & 4 \mathrm{~b} & \mathrm{CH} & \mathrm{CONH}_{2} & 2.3 & 0.3 & 0.13 \\ 4 & 3 \mathrm{~b} & \mathrm{CH} & \mathrm{SO}_{2} \mathrm{Me} & 0.04 & 7.9 & 197\end{array}$

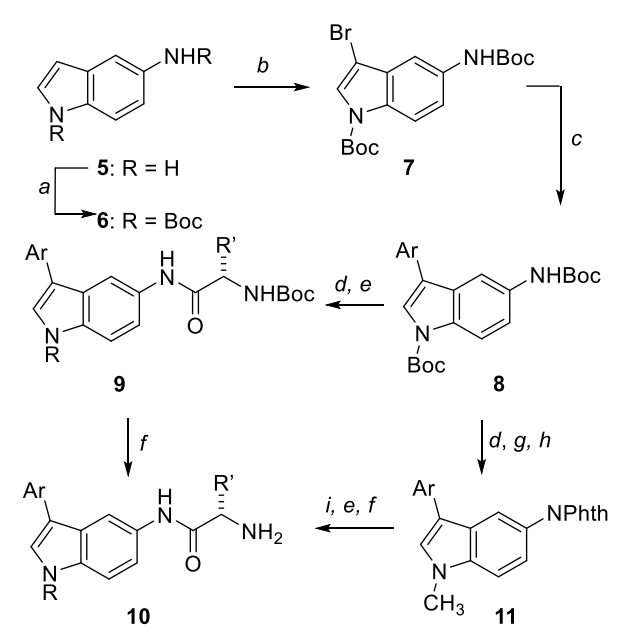

Scheme 1. General synthetic route for preparing indole-based $\mathrm{PRMT}_{4}$ inhibitors. Reagents and conditions: (a) $\mathrm{Boc}_{2} \mathrm{O}$, THF; (b) NBS, THF; (c) $\mathrm{ArB}(\mathrm{OR})_{2}, \mathrm{~K}_{2} \mathrm{CO}_{3}, \mathrm{Pd}\left(\mathrm{PPh}_{3}\right)_{4}$ or $\mathrm{Pd}(\mathrm{dppf}) \mathrm{Cl}_{2} \cdot \mathrm{CH}_{2} \mathrm{Cl}_{2}$, THF: $\mathrm{H}_{2} \mathrm{O}$ (3:1), 80 ${ }^{\circ} \mathrm{C}$; (d) TFA; (e) N-Boc- amino acid, DIPEA, pyBOP, DMF (f) TFA; g) phthalic anhydride, toluene, reflux; h) $\mathrm{K}_{2} \mathrm{CO}_{3}, \mathrm{CH}_{3} \mathrm{I}, \mathrm{DMF}$, rt; i) $\mathrm{H}_{2} \mathrm{NNH}_{2}$, $\mathrm{MeOH}$,

Table 2. Biological evaluation of sulfone and sulfonamide series

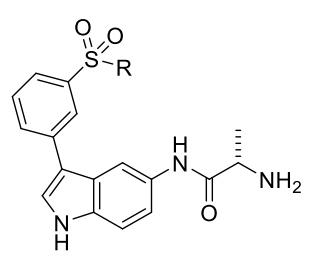

$\begin{array}{llll}\text { Entry } & \text { Comp. } & \mathrm{R} & \begin{array}{l}\mathrm{PRMT}_{4} \\ \left(\mathrm{IC}_{50} \mu \mathrm{M}\right)\end{array} \\ \mathbf{1} & 3 \mathrm{~b} & \mathrm{Me} & 0.04 \\ \mathbf{2} & 12 & \mathrm{NMe}_{2} & 0.01 \\ \mathbf{3} & 13 & \mathrm{NEt}_{2} & 3.5 \\ 4 & 14 & \mathrm{~N}\left(\mathrm{CH}_{2}\right)_{5} & 0.89 \\ \mathbf{5} & 15 & \mathrm{~N}\left(\mathrm{CH}_{2}\right)_{4} & 0.18 \\ \mathbf{6} & 16 & \mathrm{iPr}_{2} & 0.01 \\ 7 & 17 & \mathrm{C}\left(\mathrm{CH}_{2}\right)_{4} & 3.3\end{array}$

To further probe the effect of modifications at the meta-position in indole $3 \mathrm{~b}$, a series of sulfones and sulfonamides was prepared in a straightforward manner as summarized in Scheme 1 and Table 2 . Here, we found that exchanging the methyl sulfone for a dimethyl sulfonamide (e.g. compound 12, entries 1 and 2) resulted in a 2.5-fold gain in PRMT4 activity $\left(\mathrm{IC}_{50}\right.$ $=0.014 \mu \mathrm{M}$ ) and a 3 -fold improvement in selectivity (650-fold). This result indicated that a more lipophilic dimethyl sulfonamide better exploits the hydrophobic binding pocket in the $\mathrm{PRMT}_{4}$ active site. However, this modification was accompanied by a loss in potency when more sterically hindered sulfonamides were examined. For example, the diethyl sulfonamide 13 (entry $3, \mathrm{IC}_{50}=3.5 \mu \mathrm{M}$ ) and cyclic sulfonamide 14 (entry $\left.4, \mathrm{IC}_{50}=0.89 \mu \mathrm{M}\right)$ proved to be less active against PRMT 4 . While the smaller five membered ring sulfonamide 15 (entry 5) was tolerated $\left(\mathrm{IC}_{50}=0.18 \mu \mathrm{M}\right)$, this compound was still less potent and selective than original methyl sulfone $3 \mathrm{~b}$. Several analogues of the methyl sulfone 3 b were also synthesized and it was found that the isopropyl sulfone 16 (entry 6) was a potent $\mathrm{PRMT}_{4}$ inhibitor $\left(\mathrm{IC}_{50}=0.03 \mu \mathrm{M}\right)$. Here again, a similar trend to that seen with sulfonamides was observed. Specifically, increasing the size of the alkyl sulfone led to a significant loss in potency (e.g., $17 ; \mathrm{IC}_{50}=3.3 \mu \mathrm{M}$ ). This data suggested that the hydrophobic binding pocket in PRMT4 could not accommodate groups larger than the dimethyl sulfonamide or isopropyl sulfone. As a result, dimethyl sulfonamide 12 (entry 2) and isopropyl sulfone 16 (entry 6) were selected as the lead molecules for further optimization.

Having identified that both the sulfone and sulfonamide confer excellent PRMT4 activity and selectivity, we then turned 
bioRxiv preprint doi: https://doi.org/10.1101/2020.11.17.387233; this version posted November $18,2020$. The copyright holder for this preprint (which was not certified by peer review) is the author/funder, who has granted bioRxiv a license to display the preprint in perpetuity. It is made available under aCC-BY-NC-ND 4.0 International license.

our attention towards the amino acid side of the molecule. Here, we aimed to increase lipophilicity to improve cellular acid SAR study on the isopropyl sulfone and dimethyl sulfonamide scaffolds was undertaken through the synthesis of com-

\section{Table 3. SAR of the amino acid moiety}<smiles>[R3]NCCNc1ccc2[nH]cc(-c3cccc(S([R1])(=O)=O)c3)c2c1</smiles>

\begin{tabular}{|c|c|c|c|c|c|c|c|c|c|c|c|}
\hline Entry & Comp. & $\mathrm{R}_{1}$ & $\mathrm{R}_{2}$ & $\mathrm{R}_{3}$ & $\begin{array}{l}\mathrm{PRMT}_{4} \\
\left(\mathrm{IC}_{5 \mathrm{o}} \mu \mathrm{M}\right)\end{array}$ & Entry & Comp. & $\mathrm{R}_{1}$ & $\mathrm{R}_{2}$ & $\mathrm{R}_{3}$ & $\begin{array}{l}\mathrm{PRMT}_{4} \\
\left(\mathrm{IC}_{50}\right. \\
\mu \mathrm{M})\end{array}$ \\
\hline $\mathbf{1}$ & $18 a$ & $\mathrm{Me}$ & $\left(\mathrm{CH}_{2}\right)_{3}$ & $\left(\mathrm{CH}_{2}\right)_{3}$ & $>10$ & 7 & $18 \mathrm{~d}$ & $\mathrm{NMe}_{2}$ & $\left(\mathrm{CH}_{2}\right)_{2}$ & $\left(\mathrm{CH}_{2}\right)_{2}$ & 0.11 \\
\hline 2 & $19 a$ & $\mathrm{Me}$ & $\mathrm{N} / \mathrm{A}$ & $\mathrm{H}$ & $>10$ & 8 & $18 \mathrm{e}$ & $\mathrm{NMe}_{2}$ & $\mathrm{Me}$ & $\mathrm{Me}$ & 4.2 \\
\hline 3 & $19 \mathrm{~b}$ & $\mathrm{Me}$ & N/A & $\mathrm{Me}$ & 27 & 9 & $18 \mathrm{f}$ & $\mathrm{NMe}_{2}$ & $\mathrm{H}$ & $\mathrm{H}$ & 0.01 \\
\hline 4 & $18 \mathrm{~b}$ & $\mathrm{iPr}_{2}$ & $\mathrm{Me}$ & $\mathrm{Me}$ & $>10$ & 10 & $18 \mathrm{~g}$ & $\mathrm{NMe}_{2}$ & $\mathrm{Me}_{2}$ & $\mathrm{H}$ & 0.33 \\
\hline 5 & $18 \mathrm{c}$ & $\mathrm{iPr}_{2}$ & $\left(\mathrm{CH}_{2}\right)_{2}$ & $\left(\mathrm{CH}_{2}\right)_{2}$ & 0.6 & 11 & $18 \mathrm{~h}$ & $\mathrm{NMe}_{2}$ & $\left(\mathrm{CH}_{2}\right)_{2}$ & $\mathrm{H}$ & $>10$ \\
\hline
\end{tabular}

permeability and perhaps potency and selectivity. With this in mind, we chose the methyl sulfone as our starting point for the amino acid structure activity relationship study. We probed the size of the amino acid with the L-proline analogue 18a (entry 1, Table 3) and found this compound was not active (PRMT4 $\left.\mathrm{IC}_{50}>10 \mu \mathrm{M}\right)$. The ethyl amine and $N$-methyl ethyl amine 19a and 19b, respectively, were also synthesized based on the common use of ethyl amine in PRMT inhibitors ${ }^{16,17,22}$. Unfortunately, in both cases we observed a significant loss in potency ( $\mathrm{PRMT}_{4} \mathrm{IC}_{50}>10 \mu \mathrm{M}$ in both cases). A similar amino

At this point, we examined the cell permeability of the most promising compounds $3 \mathrm{~b}, 12,16$ and $18 \mathrm{~d}$. The results of the Caco-2 assay are summarized in Figure 3. From the series methyl sulfone 3 b, dimethyl sulfonamide 12 and isopropyl sulfone 16 , the dimethyl sulfonamide proved to be the most permeable $(2.13 \mathrm{~nm} / \mathrm{s}$ for $12 \mathrm{vs} 0.23 \mathrm{~nm} / \mathrm{s}$ for $3 \mathrm{~b})$. Disappointingly, replacement of alanine for azetidine in an effort to reduce $\mathrm{H}$-bond donors, increase lipophilicity and enhance cellular permeability of the compounds increased the efflux ratio by $\sim 3$ fold (Figure 3). Based on this data we further explored a series of analogues that incorporated the dimethyl sulfonamide core. pounds 18b-18c and 18d-18h, respectively (entries 4-11, Table 3). Here, we examined methylation and incorporation of an azetidine for the isopropyl sulfone and in the case of the sulfonamide, we investigated incorporation of an azetidine,

methylation, glycine incorporation, "symmetric" dimethylation and cyclopropanation. In the case of the isopropyl sulfone, each modification resulted in a decrease in PRMT 4 activity $\left(\mathrm{PRMT}_{4} \mathrm{IC}_{50}=0.6\right.$ to $\left.>10 \mu \mathrm{M}\right)$. In general, the dimethyl sulfonamide analogues $\mathbf{1 8 d - 1 8 h}$ were more potent. In particular, the glycine analogue $\mathbf{1 8 f}$ proved to be a low nM inhibitor of PRMT4 $\left(\mathrm{IC}_{50}=0.01 \mu \mathrm{M}\right)$.

\section{Table 4. Evaluation of methylated indole derivatives}

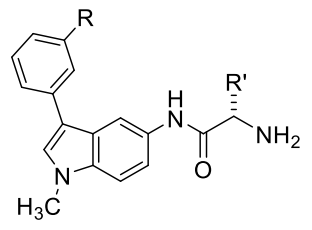

$\begin{array}{lllll}\text { Entry } & \text { Comp. } & \mathrm{R} & \mathrm{R}^{\prime} & \begin{array}{l}\mathrm{PRMT}_{4} \\ \left(\mathrm{IC}_{50} \mu \mathrm{M}\right)\end{array} \\ \mathbf{1} & 20 & \mathrm{SO}_{2} \mathrm{NMe}_{2} & \mathrm{Me} & <0.002 \\ \mathbf{2} & 21 & \mathrm{SO}_{2} \mathrm{NMe}_{2} & \mathrm{CH}_{2} \mathrm{~F} & 0.18 \\ 3 & 22 & \mathrm{CONMe}_{2} & \mathrm{Me} & \mathbf{0 . 2 2}\end{array}$


bioRxiv preprint doi: https://doi.org/10.1101/2020.11.17.387233; this version posted November 18, 2020. The copyright holder for this preprint (which was not certified by peer review) is the author/funder, who has granted bioRxiv a license to display the preprint in perpetuity. It is made available under aCC-BY-NC-ND 4.0 International license.

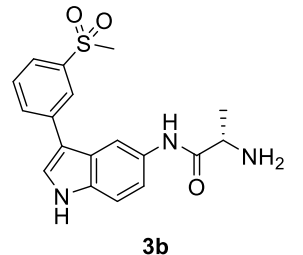

Caco-2: $0.23 \mathrm{~nm} / \mathrm{s}(34)$

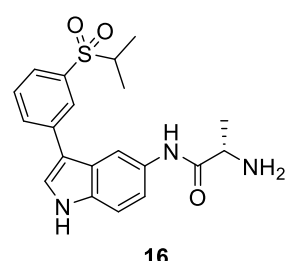

Caco-2: $1 \mathrm{~nm} / \mathrm{s}(9)$

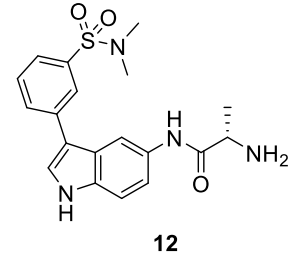

Caco-2: $2.13 \mathrm{~nm} / \mathrm{s}(12)$

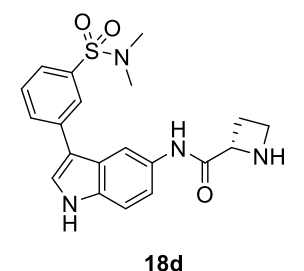

Caco-2: $2 \mathrm{~nm} / \mathrm{s}(30)$
Figure 3. Caco-2 results for key compounds (efflux ratio in parenthesis)

As the co-crystal structure of indazole za bound to PRMT6 indicated that the aryl ring was largely solvent exposed, we next focused on modifications aimed to increase lipophilicity using the most potent dimethylsulfonamide core. Thus, analogues 20-22 were synthesized as summarized in Scheme 1. Of this series, the $\mathrm{N}-\mathrm{Me}$ indole 20 proved to be the most potent and selective compound (PRMT $4 \mathrm{IC}_{50}=<2 \mathrm{nM}$, >500-fold selectivity against PRMT6, Table 4, entry 1 ). In order to assess the effect of attenuating the basicity of the free amine on membrane permeability, the fluoroalanine 21 was synthesized $^{23}$ (entry 2, Table 4), which unfortunately proved to be $\sim 10$-fold less potent and selective. To further reduce the total polar surface area of the molecule, we examined the dimethyl amide 22, however, this analogue proved also to be less active and selective. In summary, the $\mathrm{N}$-methylated derivative 20 is the most potent compound of our series against PRMT4, indicating that H-bonding of the indole-NH to Asn 162 is by far less relevant for $\mathrm{PRMT}_{4}$ binding, while it is important for the charge-reinforced interaction to Glu 59 in PRMT6. Hence, methylation of the indole- $\mathrm{N}$ is a key driver to achieve selectivity against PRMT6.

\section{Chemistry}

The general preparation of compounds described herein was performed as shown in Scheme 1, starting from commercially available 5-aminoindole 5. Boc Protection of the amine and indole nitrogen atoms ${ }^{24}$ gave the bis-Boc-protected compound 6, which was exposed to NBS to furnish the corresponding 3-brominated product $7^{25}$. This later material then engaged in a Suzuki reaction ${ }^{26}$ with a suitable meta-substituted sulfone or sulfonamide derivative. Following the Suzuki reaction, global deprotection was accomplished by treatment with trifluoroacetic acid. The 5-amino group was then coupled to a Boc-protected amino acid using pyBOP coupling conditions ${ }^{27,28}$. Finally, deprotection of the amino acid using trifluoroacetic acid yielded the desired indole analogues as their corresponding trifluoroacetate salts. In the case of analogues 20-22, prior to amino acid coupling, the 5 -amino group was protected as a phthalyl group and the indole nitrogen was methylated using methyl iodide and potassium carbonate (11, Scheme 1). Deprotection of the phthalimide was effected by treatment with hydrazine and the free amine was then carried through a similar sequence of steps as described above (i.e., peptide coupling and Boc-deprotection).

\section{Evaluation of cellular activity of PRMT4 inhibitors}

$\mathrm{PRMT}_{4}$ has been shown to methylate $\mathrm{BAF}_{155}$ at R1064 ${ }^{13}$. We have evaluated key compounds $\mathbf{3} \mathbf{b}, \mathbf{2 0}$ and $\mathbf{2 2}$, however, none of the compounds showed significant reduction in BAF155 methylation when tested up to $100 \mu \mathrm{M}$ ( $48 \mathrm{~h}$ exposure in HEK293 cells), while methylation of BAF 155 was abrogated by $2 \mu \mathrm{M}$ of the PRMT 4 selective inhibitor TP-64 (Figure 4 ). The absence of any significant cellular activity of compound $\mathbf{3 b}$ can be attributed to its poor permeability. Even though compounds 20 and 22 were expected to show enhanced permeability because of reduced H-bond donors (20 and 22) and reduced polar surface area (22), the absence of cellular activity indicates these changes were not sufficient to increase the cellular permeability of these series of compounds.

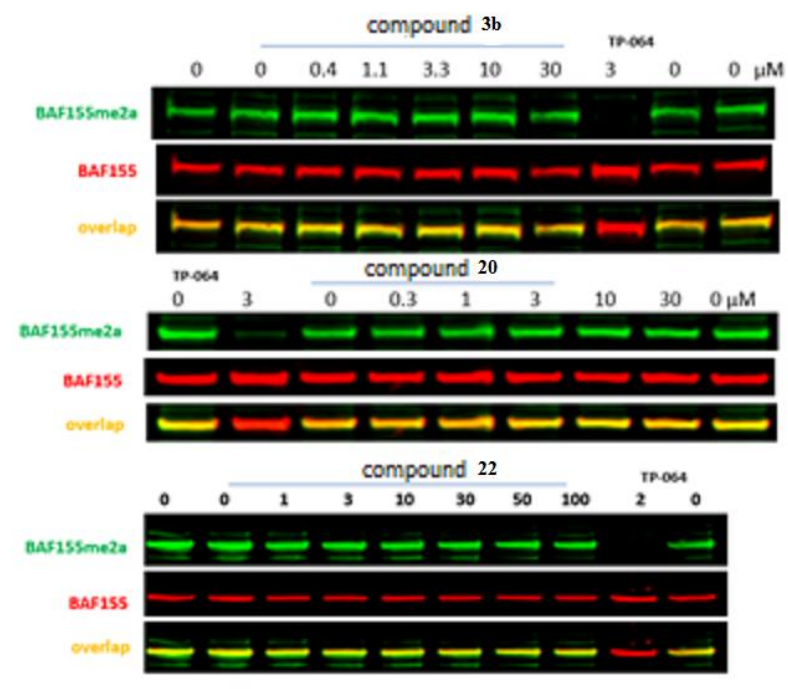

Figure 4. Effect of PRMT4 dependent BAF155 asymmetric dimethylation in HEK293T cells upon treatment with compounds $3 b, 20$ and 22 up to $100 \mu \mathrm{M}$ over 2 days.

In conclusion, we report the design, synthesis and evaluation of a new series of 3-arylindole alanine-based PRMT4 inhibitors that are both potent and selective over the closely related PRMT6. We demonstrate that selectivity for PRMT4 can be achieved by carefully tuning the substitution pattern of the aromatic ring and that amino acid substitutions are not welltolerated. Furthermore, methylation of the indole nitrogen resulted in a potent and selective in-vitro PRMT4 inhibitor. Despite these efforts, none of the compounds described herein achieved on-target effects in cells. Nonetheless, these indoles represent a new chemotype for further development of cell active $\mathrm{PRMT}_{4}$ inhibitors, which will deepen our understanding of the intricate biology of PRMT 4 and may engender the design of new anti-tumor PRMT4-selective inhibitors. These compounds could possibly be used as handles to develop PROTACs to degrade PRMT4 selectively. Linker attachment and $\mathrm{E}_{3}$ antagonist components used for developing PROTACs 

available under aCC-BY-NC-ND 4.0 International license.

will likely influence the cellular permeability of the PROTAC compounds and therefore the poor cellular permeability of our PRMT 4 inhibitors are not likely to be a hinderance for cell active PROTAC development.

\section{General Chemistry}

All reagents and starting materials were purchased from Sigma Aldrich, TCI, Alfa Aesar, CarboSynth, and AK Sci and were used without further purification. Dichloromethane was distilled from $\mathrm{CaH}_{2}$ and stored under nitrogen, THF was distilled from sodium wire/benzophenone ketyl radical and stored under nitrogen. Column chromatography was carried out with 230-400 mesh silica gel (E. Merck, Silica Gel 6o). Concentration and removal of trace solvents was done via a Buchi rotary evaporator using acetone-dry-ice condenser and a Welch vacuum pump. Nuclear magnetic resonance (NMR) spectra were recorded using deuterochloroform $\left(\mathrm{CDCl}_{3}\right)$, deuteromethanol $\left(\mathrm{CD}_{3} \mathrm{OD}\right)$, deuteroacetonitrile $\left(\mathrm{CD}_{3} \mathrm{CN}\right)$ or deuterodimethyl sulfoxide (DMSO- $\mathrm{d}_{6}$ ) as the solvent. Signal positions $(\delta)$ are given in parts per million from tetramethylsilane $(\delta \mathrm{o})$ and were measured relative to the signal of the solvent ('H NMR: $\mathrm{CDCl}_{3}: \delta 7.26 ; \mathrm{CD}_{3} \mathrm{OD}: \delta 3.31 ; \mathrm{CD}_{3} \mathrm{CN}$ : $\delta 1.96$; DMSO$\mathrm{d}_{6}: \delta 2.50 ;{ }^{13} \mathrm{C} \mathrm{NMR:} \mathrm{CDCl}_{3}: \delta 77.16 ; \mathrm{CD}_{3} \mathrm{OD}: \delta$ 49.00; $\mathrm{CD}_{3} \mathrm{CN}$ : $\left.\delta 1.32 ; \mathrm{DMSO}-\mathrm{d}_{6}: 39.5\right)$. Coupling constants ( $J$ values) are given in Hertz $(\mathrm{Hz})$ and are reported to the nearest $0.1 \mathrm{~Hz}$. ${ }^{1} \mathrm{H}$ NMR spectral data are tabulated in the order: multiplicity (s, singlet; d, doublet; t, triplet; q, quartet; sept, septet; m, multiplet; br, broad), coupling constants, number of protons. NMR spectra were recorded on a Bruker Avance 6oo equipped with a QNP or TCI cryoprobe (6oo MHz), Bruker 400 (40o MHz) or Bruker $500(500 \mathrm{MHz})$. High performance liquid chromatography (HPLC) analysis was performed on an Agilent 1100 HPLC, equipped with a variable wavelength UV-Vis detector. Highresolution mass spectrometry was performed on an Agilent 6210 TOF LC/MS.

\section{General Procedures}

General Procedure A: Suzuki-Miyaura Coupling. A pressure vial charged with a stir bar, bromoindole 7 (1.o equiv.), boronic acid or ester (1.o - 1.6 equiv.), $\mathrm{K}_{2} \mathrm{CO}_{3}$ (3.o equiv.), and $\mathrm{Pd}\left(\mathrm{PPh}_{3}\right)_{4}$ or $\mathrm{Pd}(\mathrm{dppf}) \mathrm{Cl}_{2} \mathrm{CH}_{2} \mathrm{Cl}_{2}$ (o.10 equiv.) was placed under vacuum and then filled with nitrogen. A mixture of degassed THF and $\mathrm{H}_{2} \mathrm{O}$ (o.o9 M THF- $\mathrm{H}_{2} \mathrm{O}_{3}$ :1 unless otherwise indicated) was then added and the resulting mixture was stirred under an atmosphere of nitrogen at $80^{\circ} \mathrm{C}$ for 18 hours or until the reaction was complete as monitored by TLC analysis. The reaction mixture was then cooled to room temperature and concentrated under reduced pressure. The residue was then dissolved in EtOAc and washed with saturated aqueous $\mathrm{NaHCO}_{3}$ and brine. The organic layer was dried over $\mathrm{MgSO}_{4}$ and concentrated to afford the crude aryl-indole product. Purification of the crude product by flash chromatography (silica gel, $\mathrm{Et}_{2} \mathrm{O}$ or EtOAc and hexanes) afforded the pure coupled product.

General Procedure B: Amide Coupling and Deprotection. To a stirred solution of the aryl-indole intermediate (1.0 equiv.) in dry dimethylformamide (DMF) (o.1 M) at room temperature was added $N, N$-diisopropylethylamine (DIPEA) (5 equiv.), followed by benzotriazol-1-yl-oxytripyrrolidinophosphonium hexafluorophosphate (PyBOP) (1-2 equiv.) and the protected amino acid (1-2 equiv.). The resulting solution was stirred at room temperature until completion of the reaction as monitored by TLC. The reaction mixture was then diluted with saturated aqueous $\mathrm{NaHCO}_{3}$ and extracted with EtOAc (3 times). The combined organic layers were washed with saturated aqueous $\mathrm{LiCl}$ (3 times), dried over $\mathrm{MgSO}_{4}$, and concentrated to afford the crude product (brown gum), which was used directly in the next step without further purification. The crude coupled product was dissolved in TFA (neat, o.1 M) and stirred at room temperature until the reaction was complete as monitored by TLC analysis. Purification of the crude indole by RP-HPLC (using a SiliCycle SiliaChrom dtC18 semipreparative column $(5 \mu \mathrm{m}, 100 \AA \AA, 10 \times 250 \mathrm{~mm})$ with a flow rate of 5 $\mathrm{mL} / \mathrm{min}$ eluting with solvent (A: $0.1 \%$ TFA in $\mathrm{H}_{2} \mathrm{O}$ B: $0.1 \%$ TFA in ACN) on gradients of $2 \rightarrow 30 \%$, or $2 \rightarrow 100 \%$ solvent $B$ over 15 minutes as indicated afforded the final compounds.

General Procedure C: Synthesis of Sulfonamides. To a stirred solution of amine (1.05 equiv.) in dry pyridine (0.2 M) at $\mathrm{o}^{\circ} \mathrm{C}$ was added dropwise (for liquids) or in small portions (for solids) the sulfonyl chloride (1.o equiv.). The reaction mixture was warmed to room temperature and stirred until the reaction was complete as monitored by TLC analysis. The reaction mixture was then concentrated under reduced pressure and the residue was dissolved in EtOAc and washed with 0.5 $\mathrm{M} \mathrm{HCl}$ (2 times). The organic layer was then dried over $\mathrm{MgSO}_{4}$, filtered and concentrated to afford the sulfonamide. The sulfonamide was used in subsequent reactions without further purification.

General Procedure D: Boronic Ester Synthesis. A flask was charged with a stir bar, aryl bromide (1.o equiv.), $\mathrm{B}_{2} \mathrm{pin}_{2}$ (1.0 equiv.), $\mathrm{NaHCO}_{3}$ (2.50 equiv.), and $\mathrm{Pd}(\mathrm{ddpf}) \mathrm{Cl}_{2}$ (o.o5 equiv.). The flask was then placed under vacuum and filled with nitrogen. Degassed DMSO (o.2 M) was added to the reaction vessel, and the reaction mixture was stirred under an atmosphere of nitrogen at $80{ }^{\circ} \mathrm{C}$ for 18 hours or until the reaction was complete as monitored by TLC analysis. The reaction mixture was then cooled to room temperature and diluted with equal parts $\mathrm{H}_{2} \mathrm{O}$ and EtOAc, then filtered through Celite and the Celite was rinsed with EtOAc. The filtrate was then washed with $\mathrm{H}_{2} \mathrm{O}$ and brine, dried over $\mathrm{MgSO}_{4}$ and concentrated to afford the crude product. Purification of the crude product by flash chromatography (silica gel, $\mathrm{Et}_{2} \mathrm{O}$ or EtOAc and hexanes) afforded the aryl-boronic ester.

General Procedure E: Sulfone Synthesis from Thiophenol Precursors. A stirred solution of substituted thiophenol (1 equiv.), $\mathrm{K}_{2} \mathrm{CO}_{3}$ (1.4 equiv.), and secondary bromoalkane (1.2 equiv.) in dry acetone (0.3 M), was stirred under nitrogen at reflux until completion of the reaction was observed by TLC (ca. 18 hours). The reaction mixture was cooled to room temperature, diluted with $\mathrm{H}_{2} \mathrm{O}$, and extracted with $\mathrm{Et}_{2} \mathrm{O}$ (3 times). The combined organic layers were washed with brine, dried over $\mathrm{MgSO}_{4}$, and concentrated to afford the crude aryl thioether intermediate. To a stirred solution of the crude aryl thioether intermediate (1.o equiv.) in $\mathrm{MeOH}(0.16 \mathrm{M})$ at $\mathrm{o}{ }^{\circ} \mathrm{C}$ was added oxone (potassium peroxymonosulfate) (3.0 equiv.) in $\mathrm{H}_{2} \mathrm{O}(0.5 \mathrm{M})$. The resulting white suspension was warmed to 

available under aCC-BY-NC-ND 4.0 International license.

room temperature over 2 hours and stirred at room temperature until completion was observed by TLC. The reaction mixture was then diluted with $\mathrm{H}_{2} \mathrm{O}$ and extracted with EtOAc (2 times). The combined organic layers were washed with brine, dried over $\mathrm{MgSO}_{4}$, and concentrated to afford the aryl sulfone. The aryl sulfone was used in subsequent reactions without further purification.

General Procedure F: Phthalimide protection. To a stirred solution of aminoindole intermediate (1.o equiv.) in toluene (o.2 M) was added phthalic anhydride (1.3 equiv.). The resultant solution was heated to reflux until completion of the reaction was observed by TLC. The reaction mixture was then cooled down to room temperature and concentrated under reduced pressure to afford a crude product which was used without further purification unless otherwise indicated.

General Procedure G: $N$-methylation of indole scaffolds. To a stirred solution of protected indole intermediate (1.0 equiv.) in dry dimethylformamide (DMF) (o.2 M) was added potassium carbonate ( 5 equiv.) followed by methyl iodide ( 3 equiv.). The resultant solution was stirred at $60^{\circ} \mathrm{C}$ until completion of the reaction was observed by TLC. The reaction mixture was then diluted with water and extracted with EtOAc (3 times). The combined organic layers were washed with aqueous saturated $\mathrm{LiCl}$ ( 3 times), dried over $\mathrm{MgSO}_{4}$, and concentrated to afford the crude methylated product, which was used directly in the next step without further purification unless otherwise indicated.

General procedure $\mathrm{H}$ : Phthalimide deprotection. To a stirred solution of $N$-methylindole intermediate (1.o equiv.) in methanol ( $0.06 \mathrm{M}$ ) was added hydrazine hydrate (1.3 equiv.). The resultant solution was stirred at room temperature until completion of the reaction was observed by TLC. The reaction mixture was then concentrated, diluted in dichloromethane and filtered. The resulting filtrate was concentrated under reduced pressure to afford the amino indole product, which was used directly in the next step without further purification.

General procedure I: Boc-deprotection. A solution of Bocprotected intermediate (1 eq) in TFA (o.1 M) was stirred at room temperature until completion of the reaction was observed by TLC. Concentration of the reaction mixture under reduced pressure afforded the deprotected aryl-indole product which was used without further purification unless otherwise indicated.

tert-butyl (S)-(1-((3-bromo-1H-indazol-5-yl)amino)-1-oxopropan-2-yl)carbamate (23). To a solution of (tertbutoxycarbonyl)-L-alanine (1.78 g, $9.43 \mathrm{mmol})$ in DMF (15 ml) at $21{ }^{\circ} \mathrm{C}$ was added HATU (4.30 g, $11.32 \mathrm{mmol}$ ) and $N, N$-diisopropylethylamine $(4.93 \mathrm{ml}, 28.29 \mathrm{mmol})$. The resulting solution was stirred for 10 min prior to the addition of 3 -bromo${ }_{1} \mathrm{H}$-indazol-5-amine (2.00 g, $9.43 \mathrm{mmol}$ ), and subsequent stirring for a further $16 \mathrm{~h}$. The mixture was diluted with brine (100 $\mathrm{mL})$, the aqueous layer extracted with EtOAc ( $3 \times 50 \mathrm{~mL})$, and the combined organic extracts dried with anhydrous sodium sulfate before being filtered and concentrated under reduced pressure. The residue was purified by column chromatography on silica gel (Biotage SNAP $25 \mathrm{~g}$ column, o-20\%
EtOAc/hexanes as the eluent, $25 \mathrm{CV}$ ) to afford 23 as a light yellow solid (2.53 g, 70\%). 'H NMR (400 MHz, DMSO) $\delta(\mathrm{ppm})=$ $13.34(\mathrm{~s}, 1 \mathrm{H}), 10.03(\mathrm{~s}, 1 \mathrm{H}), 8.03(\mathrm{~s}, 1 \mathrm{H}), 7.49-7.55(\mathrm{~m}, 2 \mathrm{H}), 7.07$ (br. d, $J=6.8 \mathrm{~Hz}, 1 \mathrm{H}$ ), 4.13 (br. t, $J \approx 6.8 \mathrm{~Hz}, 1 \mathrm{H}$ ), 2.28 (br d, $J$ $=7.3 \mathrm{~Hz}, 3 \mathrm{H}), 1.39(\mathrm{~s}, 9 \mathrm{H})$; LRMS = $386.2[\mathrm{M}+\mathrm{H}]^{+}$.

(S)-2-amino-N-(3-(3-(N,N-dimethylsulfamoyl)phenyl)1H-indazol-5-yl)propenamide (3a). To a solution of 23 (57.5 $\mathrm{mg}, 0.15 \mathrm{mmol}$ ) in 1-methyl-2-pyrrolidinone (NMP) (1 mL), were added subsequently [3-(dimethylsulfamoyl)phenyl]boronic acid (68.7 mg, o.30 mmol, dissolved in $0.53 \mathrm{~mL}$ 1-methyl2-pyrrolidinone),[1,1'-Bis(diphenylphosphino)ferrocene]dichloropalladium(II), complex with dichloromethane $(24.5 \mathrm{mg}$, $0.03 \mathrm{mmol}$, dissolved in $1 \mathrm{~mL} \mathrm{NMP)}$ ) and potassium carbonate $(62.2 \mathrm{mg}$, $0.45 \mathrm{mmol}$, dissolved in water $0.5 \mathrm{~mL})$. The reaction mixture was heated to $100{ }^{\circ} \mathrm{C}$ and shaken for $24 \mathrm{~h}$. The crude mixture was filtered through a pad of activated MP Alumina $\mathrm{N}$ (EcoChrom TM) and washed with NMP and concentrated under vacuum. The residue was dissolved in a TFA/ $\mathrm{CH}_{2} \mathrm{Cl}_{2}$ mixture $(1: 1,2 \mathrm{~mL})$ and shaken for $24 \mathrm{~h}$ and finally dried using a Christ-centrifuge to give $4.62 \mathrm{mg}$ of the title compound $(7 \%$ yield). LC-MS method: Instrument MS: Waters ZQ; Instrument HPLC: Waters UPLC Acquity; column: Acquity BEH $\mathrm{C}_{18}$ (Waters), $50 \mathrm{~mm}$ x 2.1mm, $1.7 \mu \mathrm{m}$; Solvent: A: $0.1 \%$ formic acid in water, solvent $\mathrm{B}$ : MeCN; Gradient: o.omin 99\% A - 1.6 min $1 \%$ A - 1.8 min $1 \% A-1.81 \min 99 \%$ A - 2.0 min $99 \%$ A; oven: 6o ${ }^{\circ} \mathrm{C}$; flow: o.80o $\mathrm{mL} / \mathrm{min}$; UV-Detection PDA 210-40onm. $\mathrm{R}_{\mathrm{t}}$ $=0.83 \mathrm{~min} ; \mathrm{LRMS}(\mathrm{ESI}): \mathrm{m} / \mathrm{z}=388[\mathrm{M}+\mathrm{H}]^{+}$

(S)-4-(5-(2-aminopropanamido)-1H-indazol-3-yl)benzamideSynthesis of benzamide (4a). Carbamate 23 (57.5 $\mathrm{mg}$, $0.15 \mathrm{mmol}$, dissolved in $1 \mathrm{~mL} \mathrm{NMP),} \mathrm{(4-car-}$ bamoylphenyl)boronic acid (49.5 $\mathrm{mg}, 0.30 \mathrm{mmol}$, dissolved in $0.53 \mathrm{~mL} \mathrm{NMP)}$ and [1,1'-Bis(diphenylphosphino)ferrocene]dichloropalladium(II), complex with dichloromethane $(24.5 \mathrm{mg}$, $0.03 \mathrm{mmol}$, dissolved in $1 \mathrm{~mL}$ NMP) along with potassium carbonate $(62.2 \mathrm{mg}, 0.45 \mathrm{mmol}$, dissolved in water $0.5 \mathrm{~mL}$ ) were heated to $100{ }^{\circ} \mathrm{C}$ and shaken for $24 \mathrm{~h}$. The microtitre plates (MTPs) were dried by Zirbus-centrifuge and then were dissolved again in $2 \mathrm{~mL}$ of a TFA/acetonitrile (1:1) mixture. The reaction mixture was further shaken for $1 \mathrm{~d}$ at room temperature. The MTPs were dried again and $2 \mathrm{~mL}$ NMP were added. The precipitated material was filtered off and purified by preparative HPLC to give $4.01 \mathrm{mg}$ of the title compound $(8 \%$ yield). LC-MS method: Instrument MS: Waters ZQ; Instrument HPLC: Waters UPLC Acquity; column: Acquity BEH $\mathrm{C}_{18}$ (Waters), $50 \mathrm{~mm} \times 2.1 \mathrm{~mm}, 1.7 \mu \mathrm{m}$; Solvent A: $0.1 \%$ formic acid in water, solvent $\mathrm{B}$ : MeCN; Gradient: o.omin 99\% A - 1.6 min $1 \%$ A - 1.8 min $1 \%$ A - 1.81 min $99 \%$ A - 2.0 min $99 \%$ A; oven: $60{ }^{\circ} \mathrm{C}$; flow: o.80o mL/min; UV-Detection PDA 210-400 $\mathrm{nm}$. $\mathrm{R}_{\mathrm{t}}$ $=0.50 \mathrm{~min} ; \mathrm{LRMS}(\mathrm{ESI}): \mathrm{m} / \mathrm{z}=324[\mathrm{M}+\mathrm{H}]^{+}$

tert-butyl 5-((tert-butoxycarbonyl)amino)-1H-indole-1carboxylate (6). To a stirred brown slurry of 5-aminoindole (1.0o g, $7.56 \mathrm{mmol}$, 1.0 equiv.) in THF ( $38 \mathrm{~mL}, 0.2 \mathrm{M})$ was added triethylamine (1.05 mL, $7.56 \mathrm{mmol}$, 1.0 equiv.), $\mathrm{Boc}_{2} \mathrm{O}$ (3.30 g, $15.1 \mathrm{mmol}$, 2.o equiv.), and DMAP (1.39 g, $11.3 \mathrm{mmol}, 1.5$ equiv.). The resulting slurry was stirred at room temperature under a nitrogen atmosphere (not sealed) for 2 days. The reaction mixture was diluted with ca. $60 \mathrm{~mL}$ of $1.0 \mathrm{M}$ aqueous $\mathrm{HCl}$ and extracted with EtOAc (2 times). The combined organic layers 
bioRxiv preprint doi: https://doi.org/10.1101/2020.11.17.387233; this version posted November 18, 2020. The copyright holder for this preprint (which was not certified by peer review) is the author/funder, who has granted bioRxiv a license to display the preprint in perpetuity. It is made available under aCC-BY-NC-ND 4.0 International license.

were dried over $\mathrm{MgSO}_{4}$ and concentrated under reduced pressure to afford the crude bis-protected aminoindole species. Purification of the crude product (silica gel, $\mathrm{Et}_{2} \mathrm{O}:$ Hexanes 1:9) provided the bis-protected aminoindole 6 (1.30 g, $52 \%)$. ${ }^{1} \mathbf{H}$ NMR: $\left(400 \mathrm{MHz}, \mathrm{CDCl}_{3}\right) \delta(\mathrm{ppm})=8.02\left(\mathrm{~d}, J=8.8 \mathrm{~Hz},{ }_{1} \mathrm{H}\right)$, $7.74(\mathrm{~s}, 1 \mathrm{H}), 7.56(\mathrm{~d}, J=3.7 \mathrm{~Hz}, 1 \mathrm{H}), 7.13(\mathrm{~d}, J=9.0 \mathrm{~Hz}, 1 \mathrm{H}), 6.65$ $-6.54(\mathrm{~m}, 1 \mathrm{H}), 6.49(\mathrm{~d}, J=3.6 \mathrm{~Hz}, 1 \mathrm{H}), 1.66(\mathrm{~s}, 9 \mathrm{H}), 1.53(\mathrm{~s}, 9 \mathrm{H})$. 13C NMR: (10o $\left.\mathrm{MHz}, \mathrm{CDCl}_{3}\right) \delta(\mathrm{ppm})=153.3,149.8,133.7,131.6$, 131.2, 126.7, 116.4, 115.4, 110.9, 107.5, 83.7, 80.4, 28.5, 28.3. HRMS: (ESI) $\mathrm{m} / \mathrm{z}$ calculated for $\mathrm{C}_{18} \mathrm{H}_{24} \mathrm{~N}_{2} \mathrm{O}_{4}[2 \mathrm{M}+\mathrm{H}]+655 \cdot 3545$, found $655 \cdot 3574$.

tert-butyl 3-bromo-5-((tert-butoxycarbonyl)amino)-1Hindole-1-carboxylate (7). To a stirred solution of 6 (1.52 g, $4.56 \mathrm{mmol}$, 1.o equiv.) in THF (13.4 mL, $0.34 \mathrm{M})$ was added NBS (852 mg, $4.79 \mathrm{mmol}$, 1.05 equiv.). The reaction vessel was wrapped in foil to exclude light and stirred at room temperature for 18 hours, after which it was concentrated under reduced pressure. The reaction residue was then dissolved in $\mathrm{Et}_{2} \mathrm{O}$ and filtered to remove the white precipitate. The filtrate was washed with saturated aqueous sodium metabisulfite, saturated aqueous $\mathrm{NaHCO}_{3}$, water, brine, dried over $\mathrm{MgSO}_{4}$, filtered, and concentrated under reduced pressure to afford 7 (1.46 g, 77 \%). ${ }^{1} \mathrm{H}$ NMR: (500 $\left.\mathrm{MHz} \mathrm{CDCl}_{3}\right) \delta(\mathrm{ppm})=8.03(\mathrm{~s}$, $1 \mathrm{H}), 7.64(\mathrm{~s}, 1 \mathrm{H}), 7.61(\mathrm{~s}, 1 \mathrm{H}), 7.25\left(\mathrm{~d}, J=8.0 \mathrm{~Hz},{ }_{1} \mathrm{H}\right), 6.60(\mathrm{~s}$, $\left.{ }_{1} \mathrm{H}\right), 1.65(\mathrm{~s}, 9 \mathrm{H}), 1.54(\mathrm{~s}, 9 \mathrm{H}) .{ }^{13} \mathrm{C}$ NMR: $\left(125 \mathrm{MHz} \mathrm{CDCl}_{3}\right) \delta$ $(\mathrm{ppm})=153.1,148.9,134.5,131.0,130.1,125.6,117.5,115.7,109.2$, 98.o, 84.4, 80.7, 28.5, 28.3. HRMS: (ESI) $\mathrm{m} / \mathrm{z}$ calculated for $\mathrm{C}_{18} \mathrm{H}_{23} \mathrm{BrN}_{2} \mathrm{O}_{4}\left[\mathrm{M}+\mathrm{NH}_{4}\right]^{+}{ }_{428.1179}$, found 428.1204 .

3-(3-(methylsulfonyl)phenyl)-1H-indol-5-amine (24). The title compound was prepared according to general procedure A using 7 (583 mg, $1.5 \mathrm{mmol}$ ), 4,4,5,5-tetramethyl-2-(3- (methylsulfonyl)phenyl)-1,3,2-dioxaborolane (400 $\mathrm{mg}, 1.5 \mathrm{mmol}$ ), $\mathrm{K}_{2} \mathrm{CO}_{3}$ (588 mg, $\left.4.3 \mathrm{mmol}\right), \mathrm{Pd}\left(\mathrm{PPh}_{3}\right)_{4}(164 \mathrm{mg}$, o.15 mmol), degassed THF $(12 \mathrm{~mL})$ and degassed water $(4 \mathrm{~mL})$. Purification by column chromatography afforded the protected coupled product, which was subsequently deprotected in TFA (15 mL) and concentrated under reduced pressure to afford the TFA salt of $\mathbf{2 4}$ as a brown solid (453 mg, 80\%). ${ }^{1} \mathrm{H}$ NMR: $(500 \mathrm{MHz}$, $\left.\mathrm{CD}_{3} \mathrm{OD}\right) \delta(\mathrm{ppm})=8.21(\mathrm{dd}, J=1.8,1.7 \mathrm{~Hz}, 1 \mathrm{H})$, 8.00 (ddd, $J=$ 7.8, 1.8, $1.4 \mathrm{~Hz}, 1 \mathrm{H}), 7.91(\mathrm{~d}, J=2.1 \mathrm{~Hz}, 1 \mathrm{H}), 7.85$ (ddd, $J=7.9$, $1.7,1.4 \mathrm{~Hz}, 1 \mathrm{H}), 7.81(\mathrm{~s}, 1 \mathrm{H}), 7.71(\mathrm{dd}, J=7.9,7.8 \mathrm{~Hz}, 1 \mathrm{H}), 7.63$ $\left(\mathrm{d}, J=8.6 \mathrm{~Hz},{ }_{1} \mathrm{H}\right), 7.22\left(\mathrm{dd}, J=8.6,2.1 \mathrm{~Hz},{ }_{1} \mathrm{H}\right), 3.19\left(\mathrm{~s},{ }_{3} \mathrm{H}\right) .{ }^{13} \mathrm{C}$ NMR: (125 MHz, CD $3 \mathrm{OD}) \delta(\mathrm{ppm})=142.8$, 138.3, 138.2, 133.1, 131.2, 127.5, 126.8, 126.1, 125.5, 124.6, 117.5, 116.8, 114.6, 114.2, 44.4. HRMS: (ESI) $\mathrm{m} / \mathrm{z}$ calculated for $\mathrm{C}_{15} \mathrm{H}_{14} \mathrm{~N}_{2} \mathrm{O}_{2} \mathrm{~S} \quad[\mathrm{M}+\mathrm{H}]^{+}$ 287.0849, found 287.0857 .

(S)-2-amino-N-(3-(3-(methylsulfonyl)phenyl)-1H-indol-5yl)propenamide $(3 \mathbf{b})$. The title compound was prepared according to general procedure B using the aryl-indole 24 (63.2 $\mathrm{mg}, 0.158 \mathrm{mmol}$ ), (tert-butoxycarbonyl)- $L$-alanine (6o mg, 0.32 mmol), PyBOP (165 mg, $0.32 \mathrm{mmol}$ ), DIPEA (o.21 mL, 0.79 $\mathrm{mmol}$ ), and dry DMF (1.6 mL). Purification by RP-HPLC (using a SiliCycle SiliaChrom dtC18 semipreparative column (5 $\mu \mathrm{m}, 100 \AA \AA, 10 \times 250 \mathrm{~mm}$ ) with a flow rate of $5 \mathrm{~mL} / \mathrm{min}$ eluting with solvent (A: $0.1 \%$ TFA in water B: o.1 \% TFA in MeCN) on a gradient of $(2 \rightarrow 100) \%$ solvent $B$ over $15 \mathrm{~min}, t R=5.68 \mathrm{~min})$ of the crude deprotected product afforded the TFA salt of $\mathbf{3 b}$ as a colorless solid (11 mg, 18\%). ${ }^{1} \mathbf{H}$ NMR: (6oo $\left.\mathrm{MHz}, \mathrm{CD}_{3} \mathrm{OD}\right)$ $\delta(\mathrm{ppm})=8.24(\mathrm{~d}, J=1.9 \mathrm{~Hz}, 1 \mathrm{H}), 8.22(\mathrm{~s}, 1 \mathrm{H}), 7.99(\mathrm{~d}, J=7.7$ $\mathrm{Hz}, 1 \mathrm{H}), 7.8 \mathrm{o}(\mathrm{d}, J=7.6 \mathrm{~Hz}, 1 \mathrm{H}), 7.7 \mathrm{o}-7.64(\mathrm{~m}, 2 \mathrm{H}), 7.45(\mathrm{~d}, J$ $=8.7 \mathrm{~Hz}, 1 \mathrm{H}), 7.33(\mathrm{dd}, J=8.7,2.0 \mathrm{~Hz}, 1 \mathrm{H}), 4.10(\mathrm{q}, J=7.0 \mathrm{~Hz}$, $\left.{ }_{1 \mathrm{H}}\right), 3.20(\mathrm{~s}, 3 \mathrm{H}), 1.63(\mathrm{~d}, J=7.1 \mathrm{~Hz}, 3 \mathrm{H}) .{ }^{13} \mathrm{C}$ NMR: $(150 \mathrm{MHz}$, $\left.\mathrm{CD}_{3} \mathrm{OD}\right) \delta(\mathrm{ppm})=169.1,142.5,139.1,136.2,132.9,132.1,131.0$, 126.4, 126.0, 126.0, 124.9, 117.2, 116.4, 113.2, 111.8, 50.9, 44.5, 17.8. HRMS: (ESI) $m / z$ calculated for $\mathrm{C}_{18} \mathrm{H}_{19} \mathrm{~N}_{3} \mathrm{O}_{3} \mathrm{~S}[\mathrm{M}+\mathrm{H}]+358.1220$, found 358.1230 .

4-(5-amino-1H-indol-3-yl)benzamide (25). The title compound was prepared according to general procedure A using 7 (454 mg, $1.10 \mathrm{mmol}$ ), para-carbamoylphenyl-boronic acid (291 $\mathrm{mg}, \quad 1.76 \mathrm{mmol}), \quad \mathrm{K}_{2} \mathrm{CO}_{3} \quad(457 \mathrm{mg}, \quad 3.31 \mathrm{mmol})$, $\mathrm{Pd}(\mathrm{ddpf}) \mathrm{Cl}_{2} \mathrm{CH}_{2} \mathrm{Cl}_{2}$ (9o mg, o.11 mmol), degassed THF/water (3:1, $20 \mathrm{~mL}$, 0.056 M). Purification by column chromatography afforded the protected coupled product, which was subsequently deprotected in TFA ( $11 \mathrm{~mL})$ and concentrated under reduced pressure to afford the TFA salt of 25 as a brown solid

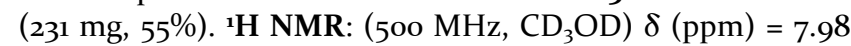
$(\mathrm{d}, J=8.4 \mathrm{~Hz}, 2 \mathrm{H}), 7.92\left(\mathrm{~d}, J=2.1 \mathrm{~Hz},{ }_{1} \mathrm{H}\right), 7.8 \mathrm{o}-7.74\left(\mathrm{~m},{ }_{3} \mathrm{H}\right)$, $7.61(\mathrm{~d}, J=8.6 \mathrm{~Hz}, 1 \mathrm{H}), 7.19\left(\mathrm{dd}, J=8.6,2.1 \mathrm{~Hz},{ }_{1} \mathrm{H}\right) .{ }^{13} \mathrm{C}$ NMR: $\left(125 \mathrm{MHz}, \mathrm{CD}_{3} \mathrm{OD}\right) \delta(\mathrm{ppm})=172.2,140.5,138.2,132.0,129.4$, $127.7,127.3,127.0,124.5,117.6,117.2,114.43,114.39$. HRMS: (ESI) $\mathrm{m} / \mathrm{z}$ calculated for $\mathrm{C}_{15} \mathrm{H}_{13} \mathrm{~N}_{3} \mathrm{O}[\mathrm{M}+\mathrm{H}]+252.1131$, found 252.1144 .

(S)-4-(5-(2-aminopropanamido)-1H-indol-3-yl)ben-

zamide (4b). The title compound was prepared according to general procedure B using the aryl-indole $25(25 \mathrm{mg}, 0.068$ $\mathrm{mmol})$, (tert-butoxycarbonyl)- $L$-alanine (17 mg, $0.072 \mathrm{mmol})$, PyBOP (43 mg, o.082 mmol), DIPEA (o.06 mL, $0.34 \mathrm{mmol}$ ), and dry DMF (o.68 mL). Purification by RP-HPLC (using a SiliCycle SiliaChrom dtC18 semipreparative column $(5 \mu \mathrm{m}, 100 \AA$, $10 \times 250 \mathrm{~mm}$ ) with a flow rate of $5 \mathrm{~mL} / \mathrm{min}$ eluting with solvent (A: $0.1 \%$ TFA in water B: $0.1 \%$ TFA in MeCN) on a gradient of $(2 \rightarrow 100) \%$ solvent $B$ over $15 \mathrm{~min}, \mathrm{tR}=4.52 \mathrm{~min})$ of the crude deprotected product afforded the TFA salt of $\mathbf{4} \mathbf{b}$ as a colorless solid (9 mg, 32\%). ${ }^{1} \mathbf{H}$ NMR: (500 MHz, CD $\left.\mathrm{OD}\right) \delta(\mathrm{ppm})=8.23$ $(\mathrm{d}, J=1.7 \mathrm{~Hz}, 1 \mathrm{H}), 7.94(\mathrm{~d}, J=8.5 \mathrm{~Hz}, 2 \mathrm{H}), 7.77(\mathrm{~d}, J=8.5 \mathrm{~Hz}$, $2 \mathrm{H}), 7.64(\mathrm{~s}, 1 \mathrm{H}), 7.43\left(\mathrm{~d}, J=8.7 \mathrm{~Hz},{ }_{1} \mathrm{H}\right), 7.32(\mathrm{dd}, J=8.7,1.7$ $\mathrm{Hz}, 1 \mathrm{H}), 4.09(\mathrm{q}, J=7.1 \mathrm{~Hz}, 1 \mathrm{H}), 1.64(\mathrm{~d}, J=7.1 \mathrm{~Hz}, 3 \mathrm{H}),{ }^{13} \mathrm{C}$ NMR: (125 MHz, CD 3 OD) $\delta(\mathrm{ppm})=172.4$, 169.0, 141.4, 136.3, 131.8, 131.4, 129.3, 127.5, 126.6, 126.0, 117.3, 117.2, 113.1, 112.5, 50.9, 17.7. HRMS: (ESI) $\mathrm{m} / \mathrm{z}$ calculated for $\mathrm{C}_{18} \mathrm{H}_{18} \mathrm{~N}_{4} \mathrm{O}_{2}[\mathrm{M}+\mathrm{H}]^{+}$ 323.1503, found 323.1477 .

3-bromo-N,N-dimethylbenzenesulfonamide (26). The title compound was prepared according to general procedure $C$, using dimethylamine (0.024 mL, $0.36 \mathrm{mmol})$, dry pyridine ( 1.8 $\mathrm{mL}$ ), and 3-bromobenzenesulfonyl chloride (o.056 mL, 0.39 mmol). Workup of the reaction mixture as detailed in general procedure $C$ afforded the aryl-sulfonamide $\mathbf{2 6}$ as a colorless oil (87 mg, $95 \%$ ). ' $\mathbf{H}$ NMR: (500 MHz, $\left.\mathrm{CDCl}_{3}\right) \delta(\mathrm{ppm})=7.73(\mathrm{dd}$, $J=1.9,1.7 \mathrm{~Hz}, 1 \mathrm{H}), 7.71$ (ddd, $J=8.0,1.9,1.0 \mathrm{~Hz}, 1 \mathrm{H}), 7.43$ (ddd, $J=7.9,1.7,1.0 \mathrm{~Hz}, 1 \mathrm{H}), 7.43(\mathrm{dd}, J=8.0,7.9 \mathrm{~Hz}, 1 \mathrm{H}), 2.74(\mathrm{~s}, 6 \mathrm{H})$

N,N-dimethyl-3-(4,4,5,5-tetramethyl-1,3,2-dioxaborolan2-yl)benzenesulfonamide (27). The title compound was prepared according to general procedure $\mathrm{D}$, using aryl-sulfonamide 26 (71 mg, $0.27 \mathrm{mmol}), \mathrm{B}_{2} \mathrm{Pin}_{2}$ (205 mg, o.81 mmol), $\mathrm{NaOAc}(88 \mathrm{mg}, 1.08 \mathrm{mmol})$ and $\mathrm{Pd}(\mathrm{dppf}) \mathrm{Cl}_{2}(22 \mathrm{mg}, 0.027$ $\mathrm{mmol})$ in degassed dioxane $(1.1 \mathrm{~mL})$. Purification of the crude 
bioRxiv preprint doi: https://doi.org/10.1101/2020.11.17.387233; this version posted November 18, 2020. The copyright holder for this preprint (which was not certified by peer review) is the author/funder, who has granted bioRxiv a license to display the preprint in perpetuity. It is made available under aCC-BY-NC-ND 4.0 International license.

material by column chromatography (EtOAc:hexanes 10:90) afforded the 3-pinacolboronate aryl-sulfonamide $\mathbf{2 7}$ as a colorless solid ( $53 \mathrm{mg}, 63 \%)$. ${ }^{\mathbf{H}} \mathbf{H}$ NMR: (500 MHz, $\left.\mathrm{CDCl}_{3}\right) \delta(\mathrm{ppm})$ $=8.20(\mathrm{dd}, J=1.9,1.1 \mathrm{~Hz}, 1 \mathrm{H}), 8.01\left(\mathrm{ddd}, J=7.5,1.5,1.1 \mathrm{~Hz},{ }_{1} \mathrm{H}\right)$, $7.86(\mathrm{ddd}, J=7.9,1.9,1.5 \mathrm{~Hz}, 1 \mathrm{H}), 7.37(\mathrm{dd}, J=7.9,7.5 \mathrm{~Hz}, 1 \mathrm{H})$, $2.72(\mathrm{~s}, 6 \mathrm{H}), 1.35(\mathrm{~s}, 12 \mathrm{H})$.

tert-butyl 5-((tert-butoxycarbonyl)amino)-3-(3-(N,N-dimethylsulfamoyl)phenyl)-1H-indole-1-carboxylate (28). The title compound was prepared according to general procedure A using 7 (49.5 mg, $0.12 \mathrm{mmol})$, 3-pinacolboronate aryl-sulfonamide 27 ( $37 \mathrm{mg}, 0.12 \mathrm{mmol}$ ), $\mathrm{K}_{2} \mathrm{CO}_{3}$ (50 mg, 0.36 $\mathrm{mmol}), \mathrm{Pd}\left(\mathrm{PPh}_{3}\right)_{4}(14 \mathrm{mg}, 0.012 \mathrm{mmol})$, degassed THF (1.0 $\mathrm{mL}$ ) and degassed water ( $0.35 \mathrm{~mL})$. Purification by column chromatography (EtOAc:hexanes 10:90) afforded $\mathbf{2 8}$ as a pale yellow solid (32 mg, 52\%). ${ }^{1} \mathbf{H}$ NMR: (500 $\mathrm{MHz} \mathrm{CDCl}_{3}$ ) $\delta$ $(\mathrm{ppm})=8.12(\mathrm{~d}, J=8.5 \mathrm{~Hz}, 1 \mathrm{H}), 8.03(\mathrm{br} \mathrm{s}, 2 \mathrm{H}), 7.85(\mathrm{dt}, J=$ $7.8,1.2 \mathrm{~Hz}, 1 \mathrm{H}), 7.76(\mathrm{br} \mathrm{d}, J=7.6 \mathrm{~Hz}, 2 \mathrm{H}), 7.64\left(\mathrm{t}, J=7.8,{ }_{1} \mathrm{H}\right)$, $7.17(\mathrm{dd}, J=8.9,1.8 \mathrm{~Hz}, 1 \mathrm{H}), 6.56$ (br s, $1 \mathrm{H}), 2.81$ (s, 6H), 1.69 $(\mathrm{s}, 9 \mathrm{H}), 1.51(\mathrm{~s}, 9 \mathrm{H})$.

(S)-2-amino-N-(3-(3-(N,N-dimethylsulfamoyl)phenyl)1H-indol-5-yl)propenamide (12). The title compound was prepared according to the general procedures I and B using the Boc-protected-aryl-indole $28(55 \mathrm{mg}, 0.106 \mathrm{mmol})$ and TFA (1.0 mL) followed by treatment with (tert-butoxycarbonyl)- $L$-alanine (24 mg, $0.13 \mathrm{mmol}$ ), PyBOP (66 mg, 0.53 mmol), DIPEA (o.13 mL, o.13 mmol), and dry DMF (1.o mL). Final Boc-deprotection of the crude mixture using TFA (1.0 $\mathrm{mL}$ ) and subsequent purification by RP-HPLC (using a SiliCycle SiliaChrom dtC18 semipreparative column $(5 \mu \mathrm{m}, 100 \AA ̊$, $10 \times 250 \mathrm{~mm}$ ) with a flow rate of $5 \mathrm{~mL} / \mathrm{min}$ eluting with solvent (A: $0.1 \%$ TFA in water B: $0.1 \%$ TFA in $\mathrm{MeCN}$ ) on a gradient of $(2 \rightarrow 100) \%$ ) solvent $B$ over $\left.15 \mathrm{~min}, t_{R}=6.2 \mathrm{~min}\right)$ afforded the TFA salt of 12 as a colorless solid ( $8 \mathrm{mg}, 19 \%)$. ${ }^{1}$ H NMR: (400 $\left.\mathrm{MHz}, \mathrm{CD}_{3} \mathrm{OD}\right) \delta(\mathrm{ppm})=8.32(\mathrm{~d}, J=1.9 \mathrm{~Hz}, 1 \mathrm{H}), 8.06($ br s, $1 \mathrm{H})$, $7.95(\mathrm{dt}, J=6.7,2.1 \mathrm{~Hz}, 1 \mathrm{H}), 7.68-7.61\left(\mathrm{~m},{ }_{3} \mathrm{H}\right), 7.45(\mathrm{~d}, J=8.8$ $\left.\mathrm{Hz},{ }_{1 \mathrm{H}}\right), 7.26(\mathrm{dd}, J=8.8,2.1 \mathrm{~Hz}, 1 \mathrm{H}), 4.08(\mathrm{q}, J=7.1 \mathrm{~Hz}, 1 \mathrm{H})$, $2.78(\mathrm{~s}, 6 \mathrm{H}), 1.63(\mathrm{~d}, J=7.1 \mathrm{~Hz}, 3 \mathrm{H}) .{ }^{13} \mathrm{C}$ NMR: $(150 \mathrm{MHz}$, $\left.\mathrm{CD}_{3} \mathrm{OD}\right) \delta(\mathrm{ppm})=168.9,138.8,136.9,136.2,132.2,132.1,130.7$, 126.6, 126.4, 125.9, 125.5, 117.2, 116.6, 113.2, 111.8, 50.89, 38.56, 17.75 HRMS: (ESI) $\mathrm{m} / \mathrm{z}$ calculated for $\mathrm{C}_{19} \mathrm{H}_{22} \mathrm{~N}_{4} \mathrm{O}_{3} \mathrm{~S}[\mathrm{M}+\mathrm{H}]^{+}$ 387.1491 , found $387.145^{2}$

3-bromo-N,N-diethylbenzenesulfonamide (29). The title compound was prepared according to general procedure $C$, using diethylamine (o.037 mL, $0.36 \mathrm{mmol})$, dry pyridine $(1.8$ $\mathrm{mL}$ ), and 3-bromobenzenesulfonyl chloride (0.056 mL, 0.39 $\mathrm{mmol}$ ). Workup of the reaction mixture as detailed in general procedure $C$ afforded the aryl-sulfonamide 29 as a colorless oil (97 mg, 92 \%). ${ }^{1} \mathbf{H}$ NMR: (50o MHz, $\mathrm{CDCl}_{3}$ ) $\delta(\mathrm{ppm})=7.95$ (dd, $J=1.9,1.6 \mathrm{~Hz}, 1 \mathrm{H}), 7.74(\mathrm{ddd}, J=7.9,1.6,1.0 \mathrm{~Hz}, 1 \mathrm{H}), 7.67$ (ddd, $J=8.0,1.9,1.0 \mathrm{~Hz}, 1 \mathrm{H}), 7.41(\mathrm{dd}, J=8.0,7.9 \mathrm{~Hz}, 1 \mathrm{H}), 3.26(\mathrm{q}, J=$ $7.2 \mathrm{~Hz}, 4 \mathrm{H}), 1.15(\mathrm{t}, J=7.2 \mathrm{~Hz}, 6 \mathrm{H})$.

N,N-diethyl-3-(4,4,5,5-tetramethyl-1,3,2-dioxaborolan-2yl)benzenesulfonamide (30). The title compound was prepared according to general procedure $\mathrm{D}$, using aryl-sulfonamide 29 (141 mg, $0.48 \mathrm{mmol}$ ), $\mathrm{B}_{2} \mathrm{Pin}_{2}(365.6 \mathrm{mg}, 1.44 \mathrm{mmol}$ ), $\mathrm{NaOAc}$ (188 mg, $1.92 \mathrm{mmol}$ ) and $\mathrm{Pd}(\mathrm{dppf}) \mathrm{Cl}_{2}$ (39 mg, 0.05 $\mathrm{mmol})$ in degassed dioxane $(1.9 \mathrm{~mL})$. Purification of the crude material by column chromatography (EtOAc:hexanes 10:90) afforded the 3-pinacolboronate aryl-sulfonamide 30 as a colorless solid (94.5 mg, $58 \%$ ). ${ }^{\mathbf{1}} \mathbf{H}$ NMR: (500 $\left.\mathrm{MHz}, \mathrm{CDCl}_{3}\right) \delta$ $(\mathrm{ppm})=8.23(\mathrm{dd}, J=2.0,1.0 . \mathrm{Hz}, 1 \mathrm{H}), 7.96(\mathrm{ddd}, J=7.4,1.3$, 1.0 $\mathrm{Hz},{ }_{1} \mathrm{H}$ ), 7.88 (ddd, $J=7.9,2.0,1.3 \mathrm{~Hz}, 1 \mathrm{H}$ ), 7.48 (dd, $J=7.9,7.4$ $\left.\mathrm{Hz},{ }_{1} \mathrm{H}\right), 3.25\left(\mathrm{q}, J=7.2 \mathrm{~Hz},{ }_{4} \mathrm{H}\right), 1.35(\mathrm{~s}, 12 \mathrm{H}), 1.14(\mathrm{t}, J=7.2 \mathrm{~Hz}$, $6 \mathrm{H})$.

tert-butyl 5-((tert-butoxycarbonyl)amino)-3-(3-(N,N-diethylsulfamoyl)phenyl)-1H-indole-1-carboxylate (31). The title compound was prepared according to general procedure A using 7 (128 mg, $0.31 \mathrm{mmol})$, 3-pinacolboronate aryl-sulfonamide 30 (104 mg, $0.31 \mathrm{mmol}), \mathrm{K}_{2} \mathrm{CO}_{3}(127 \mathrm{mg}, 0.93 \mathrm{mmol}$ ), $\mathrm{Pd}\left(\mathrm{PPh}_{3}\right)_{4}(35.5 \mathrm{mg}, 0.031 \mathrm{mmol})$, degassed THF $(2.6 \mathrm{~mL})$ and degassed water $(0.9 \mathrm{~mL})$. Purification by column chromatography (EtOAc:hexanes 10:90) afforded 31 as a pale yellow solid (100 mg, 59\%). ' ${ }^{H}$ NMR: (500 MHz, $\left.\mathrm{CDCl}_{3}\right) \delta(\mathrm{ppm})=8.14(\mathrm{br}$ $\mathrm{t}, J=8.0 \mathrm{~Hz}, 1 \mathrm{H}), 8.04(\mathrm{t}, J=1.2 \mathrm{~Hz}, 1 \mathrm{H}), 7.93(\mathrm{br} \mathrm{s}, 1 \mathrm{H}), 7.8 \mathrm{o}(\mathrm{tt}$, $J=7.8,1.2 \mathrm{~Hz}, 2 \mathrm{H}), 7.73($ br s, $1 \mathrm{H}), 7.59(\mathrm{t}, J=7.8 \mathrm{~Hz}, 1 \mathrm{H}), 7.23$ $(\mathrm{dd}, J=8.9,1.8 \mathrm{~Hz}, 1 \mathrm{H}), 6.55(\mathrm{br} \mathrm{s}, 1 \mathrm{H}), 3.32\left(\mathrm{t}, J=7.6 \mathrm{~Hz},{ }_{4} \mathrm{H}\right)$, $1.69(\mathrm{~s}, 9 \mathrm{H}), 1.52(\mathrm{~s}, 9 \mathrm{H}), 1.19(\mathrm{t}, J=7.6 \mathrm{~Hz}, 6 \mathrm{H})$.

(S)-2-amino-N-(3-(3-(N,N-diethylsulfamoyl)phenyl)-1Hindol-5-yl)propanamide (13). The title compound was prepared according to the general procedures I and B using the Boc-protected-aryl-indole 31 (50 $\mathrm{mg}, 0.091 \mathrm{mmol})$ and TFA (o.9 mL) followed by treatment with (tert-butoxycarbonyl)- $L$ alanine (20 mg, o.11 mmol), PyBOP (56 mg, o.45 mmol), DIPEA (o.08 mL, o.11 mmol), and dry DMF (o.9 mL). Final Boc-deprotection of the crude mixture using TFA (o.9 mL) and subsequent purification by RP-HPLC (using a SiliCycle SiliaChrom dtC18 semipreparative column $(5 \mu \mathrm{m}$, 100 $\mathrm{A}, 10 \mathrm{x} 250 \mathrm{~mm})$ with a flow rate of $5 \mathrm{~mL} / \mathrm{min}$ eluting with solvent (A: $0.1 \%$ TFA in water B: $0.1 \%$ TFA in MeCN) on a gradient of $(2 \rightarrow 100) \%)$ solvent $B$ over $\left.15 \mathrm{~min}, \mathrm{t}_{\mathrm{R}}=6.6 \mathrm{~min}\right)$ afforded the TFA salt of 13 as a colorless solid (6 mg, 16\%). ' $\mathbf{H}$ NMR: (400 MHz, DMSO$\left.\mathrm{d}_{6}\right) \delta(\mathrm{ppm})=11.61(\mathrm{~s}, 1 \mathrm{H}), 10.37(\mathrm{~s}, 1 \mathrm{H}), 8.27(\mathrm{~s}, 1 \mathrm{H}), 8.20(\mathrm{br} \mathrm{s}$, 2H), $7.98(\mathrm{~s}, 1 \mathrm{H}), 7.90\left(\mathrm{dt}, J=1.6,7.1 \mathrm{~Hz},{ }_{1} \mathrm{H}\right), 7.88(\mathrm{~d}, J=2.5 \mathrm{~Hz}$, $\left.{ }_{1 \mathrm{H}}\right), 7.68-7.59(\mathrm{~m}, 2 \mathrm{H}), 7.47(\mathrm{~d}, J=8.8 \mathrm{~Hz}, 1 \mathrm{H}), 7.31(\mathrm{dd}, J=8.8$, $1.6 \mathrm{~Hz}, 1 \mathrm{H}), 4.01(\mathrm{q}, J=7.0 \mathrm{~Hz}, 1 \mathrm{H}), 3.23(\mathrm{q}, J=7.0 \mathrm{~Hz}, 4 \mathrm{H}), 1.47$ $\left(\mathrm{d}, J=7.0 \mathrm{~Hz},{ }_{3} \mathrm{H}\right), 1.06(\mathrm{t}, J=7.0 \mathrm{~Hz}, 6 \mathrm{H}) .{ }_{3} \mathrm{C}$ NMR: $(150 \mathrm{MHz}$, DMSO-d 6 ) $\delta(\mathrm{ppm})=167.5,140.4,136.8,134.0,131.1$, 130.0, 129.7, 125.5, 124.4, 123.6, 123.1, 115.5, 114.1, 112.3, 109.4, 48.92, 41.83, 17.14, 14.03. HRMS: (ESI) $\mathrm{m} / \mathrm{z}$ calculated for $\mathrm{C}_{21} \mathrm{H}_{27} \mathrm{~N}_{4} \mathrm{O}_{3} \mathrm{~S}[\mathrm{M}+\mathrm{H}]^{+}$ 415.1804, found 415 .

1-((3-bromophenyl)sulfonyl)piperidine (32). The title compound was prepared according to general procedure $\mathrm{C}$, using piperidine (0.035 mL, $0.36 \mathrm{mmol})$, dry pyridine $(1.8 \mathrm{~mL})$, and 3-bromobenzenesulfonyl chloride (0.056 mL, $0.39 \mathrm{mmol}$ ). Workup of the reaction mixture as detailed in general procedure $C$ afforded the aryl-sulfonamide $\mathbf{3 2}$ as a colorless oil (106 mg, $97 \%)$. ${ }^{1}$ H NMR: (500 $\left.\mathrm{MHz} \mathrm{CDCl}_{3}\right) \delta(\mathrm{ppm})=7.90(\mathrm{dd}, J=$ $1.9,1.7 \mathrm{~Hz}, 1 \mathrm{H}), 7.71(\mathrm{ddd}, J=7.9,1.9,1.0 \mathrm{~Hz}, 1 \mathrm{H}), 7.69$ (ddd, $J=$ 7.8, 1.7, 1.0 Hz, $1 \mathrm{H}), 7.41(\mathrm{dd}, J=7.9,7.8 \mathrm{~Hz}, 1 \mathrm{H}), 3.01(\mathrm{t}, J=5.4$ $\mathrm{Hz},{ }_{4} \mathrm{H}$ ), 1.65 (quint, $\left.J=5.4 \mathrm{~Hz},{ }_{4} \mathrm{H}\right), 1.49-1.40(\mathrm{~m}, 2 \mathrm{H})$

1-((3- $(4,4,5,5$-tetramethyl-1,3,2-dioxaborolan-2-yl)phenyl)sulfonyl)piperidine (33). The title compound was prepared according to general procedure $\mathrm{D}$, using aryl-sulfonamide 32 (208 mg, $0.68 \mathrm{mmol}$ ), $\mathrm{B}_{2} \mathrm{Pin}_{2}$ (520 mg, $2.04 \mathrm{mmol}$ ), 
bioRxiv preprint doi: https://doi.org/10.1101/2020.11.17.387233; this version posted November 18, 2020. The copyright holder for this preprint (which was not certified by peer review) is the author/funder, who has granted bioRxiv a license to display the preprint in perpetuity. It is made available under aCC-BY-NC-ND 4.0 International license.

$\mathrm{NaOAc}(268 \mathrm{mg}, 2.72 \mathrm{mmol})$ and $\mathrm{Pd}(\mathrm{dppf}) \mathrm{Cl}_{2}(56 \mathrm{mg}, 0.07$ $\mathrm{mmol})$ in degassed dioxane $(2.7 \mathrm{~mL})$. Purification of the crude material by column chromatography (EtOAc:hexanes 10:90) afforded the 3-pinacolboronate aryl-sulfonamide 33 as a colorless solid (134 mg, $56 \%$ ). ${ }^{1} \mathbf{H}$ NMR: (500 $\left.\mathrm{MHz} \mathrm{CDCl}_{3}\right) \delta(\mathrm{ppm})$ $=8.17(\mathrm{dd}, J=1.8$, 1.o. Hz, $1 \mathrm{H}), 8.00(\mathrm{ddd}, J=7.4,1.4$, 1.o Hz, $1 \mathrm{H})$, 7.83 (ddd, $J=7.9,1.8,1.4 \mathrm{~Hz}, 1 \mathrm{H}), 7.52(\mathrm{t}, J=7.6 \mathrm{~Hz}, 1 \mathrm{H}), 3.00$ $(\mathrm{t}, J=5.4 \mathrm{~Hz}, 4 \mathrm{H}), 1.65$ (quint, $J=5.4 \mathrm{~Hz}, 4 \mathrm{H}), 1.45^{-1.39}(\mathrm{~m}, 2 \mathrm{H})$, $1.35(\mathrm{~s}, 12 \mathrm{H})$.

tert-butyl 5-((tert-butoxycarbonyl)amino)-3-(3-(piperidin-1-ylsulfonyl)phenyl)-1H-indole-1-carboxylate (34). The title compound was prepared according to general procedure A using 7 (117 mg, $0.29 \mathrm{mmol})$, 3-pinacolboronate arylsulfonamide 33 (100 $\mathrm{mg}, 0.29 \mathrm{mmol}), \mathrm{K}_{2} \mathrm{CO}_{3}(118 \mathrm{mg}, 0.87$ $\mathrm{mmol}), \mathrm{Pd}\left(\mathrm{PPh}_{3}\right)_{4}(33 \mathrm{mg}, 0.029 \mathrm{mmol})$, degassed THF $(2.5$ $\mathrm{mL})$ and degassed water (o.8 mL). Purification by column chromatography (EtOAc:hexanes 10:90) afforded 34 as a pale yellow solid (110 mg, 68\%). 'H NMR: (500 $\left.\mathrm{MHz}, \mathrm{CDCl}_{3}\right) \delta$ $(\mathrm{ppm})=8.13(\mathrm{br} \mathrm{d}, J=8.9 \mathrm{~Hz}, 1 \mathrm{H}), 8.00(\mathrm{t}, J=1.7 \mathrm{~Hz}, 1 \mathrm{H}), 7.98$ (br s, $1 \mathrm{H}), 7.85(\mathrm{dt}, J=7.8,1.5 \mathrm{~Hz}, 1 \mathrm{H}), 7.74$ (br s, $1 \mathrm{H}), 7.72(\mathrm{dt}$, $J=7.9,1.7 \mathrm{~Hz}, 1 \mathrm{H}), 7.62(\mathrm{t}, J=7.8 \mathrm{~Hz}, 1 \mathrm{H}), 7.20(\mathrm{dd}, J=8.9,2.1$ $\mathrm{Hz}, 1 \mathrm{H}), 6.56($ br s, $1 \mathrm{H}), 3.08\left(\mathrm{t}, J=5.3 \mathrm{~Hz},{ }_{4} \mathrm{H}\right), 1.69(\mathrm{~s}, 9 \mathrm{H}), 1.19$ (quint, $J=5.3 \mathrm{~Hz}, 4 \mathrm{H}), 1.52(\mathrm{~s}, 9 \mathrm{H}), 1.49-1.42(\mathrm{~m}, 2 \mathrm{H})$.

(S)-2-amino-N-(3-(3-(piperidin-1-ylsulfonyl)phenyl)-1Hindol-5-yl)propanamide (14). The title compound was prepared according to the general procedures I and B using the aryl-indole 34 (49 mg, o.o88 mmol) and TFA (o.9 mL) followed by treatment with (tert-butoxycarbonyl)- $L$-alanine (20 $\mathrm{mg}, 0.11 \mathrm{mmol}$ ), PyBOP (55 mg, $0.44 \mathrm{mmol}$ ), DIPEA (0.08 mL, $0.11 \mathrm{mmol})$, and dry DMF (0.9 mL). Final Boc-deprotection of the crude mixture using TFA (o.9 mL) and subsequent purification by RP-HPLC (using a SiliCycle SiliaChrom dtC18 semipreparative column $(5 \mu \mathrm{m}, 100 \AA$, $10 \times 250 \mathrm{~mm})$ with a flow rate of $5 \mathrm{~mL} / \mathrm{min}$ eluting with solvent (A: $0.1 \%$ TFA in water B: 0.1 $\%$ TFA in $\mathrm{MeCN})$ on a gradient of $(2 \rightarrow 100) \%)$ solvent $\mathrm{B}$ over $\left.15 \mathrm{~min}, \mathrm{t}_{\mathrm{R}}=6.7 \mathrm{~min}\right)$ afforded the TFA salt of 14 as a colorless solid (7 mg, 18\%). ${ }^{1} \mathrm{H}$ NMR: (400 $\left.\mathrm{MHz}, \mathrm{CD}_{3} \mathrm{CN}\right) \delta 9.69\left(\mathrm{~s},{ }_{1} \mathrm{H}\right)$, $9.06(\mathrm{~s}, 1 \mathrm{H}), 8.28(\mathrm{~s}, 1 \mathrm{H}), 8.00(\mathrm{~d}, J=2.0 \mathrm{~Hz}, 1 \mathrm{H}), 7.92(\mathrm{~d}, J=7.6$ $\left.\mathrm{Hz},{ }_{1} \mathrm{H}\right), 7.7 \mathrm{O}-7.58\left(\mathrm{~m},{ }_{3} \mathrm{H}\right), 7.48\left(\mathrm{~d}, J=8.7 \mathrm{~Hz},{ }_{1} \mathrm{H}\right), 7.27(\mathrm{dd}, J$ $=8.6,2.0 \mathrm{~Hz}, 1 \mathrm{H}), 4.19(\mathrm{q}, J=7.0 \mathrm{~Hz}, 1 \mathrm{H}), 3.03(\mathrm{t}, J=5.7 \mathrm{~Hz}$, $\left.{ }_{4} \mathrm{H}\right), 1.64$ (quint, $\left.J=5.7 \mathrm{~Hz},{ }_{4} \mathrm{H}\right), 1.59\left(\mathrm{~d}, J=7.0 \mathrm{~Hz},{ }_{3} \mathrm{H}\right), 1.45^{-}$ $1.37(\mathrm{~m}, 2 \mathrm{H})$. ${ }^{13} \mathrm{C}$ NMR: (150 MHz, DMSO-d 6$) \delta(\mathrm{ppm})=167.7$, 137.0, 136.0, 134.1, 131.4, 130.6, 130.0, 125.9, 124.5, 124.4, 124.0, $115.5,114.1,112.5,109.3,49.11,46.90,24.82,22.98,17.39$. HRMS: (ESI) $\mathrm{m} / \mathrm{z}$ calculated for $\mathrm{C}_{22} \mathrm{H}_{27} \mathrm{~N}_{4} \mathrm{O}_{3} \mathrm{~S}[\mathrm{M}+\mathrm{H}]^{+} 427.1804$, found 427.1753.

1-((3-bromophenyl)sulfonyl)pyrrolidine (35). The title compound was prepared according to general procedure $\mathrm{C}$, using pyrrolidine (0.27 mL, $3.3 \mathrm{mmol})$, dry pyridine $(16.0 \mathrm{~mL})$, and 3-bromobenzenesulfonyl chloride (80o $\mathrm{mg}, 3.1 \mathrm{mmol}$ ). Workup of the reaction mixture as detailed in general procedure $\mathrm{C}$ afforded the aryl-sulfonamide 35 as a colorless oil (710 mg, $78 \%$ ). ${ }^{\mathbf{H}} \mathbf{H}$ NMR: $\left(500 \mathrm{MHz}, \mathrm{CDCl}_{3}\right) \delta(\mathrm{ppm})=7.97(\mathrm{dd}, J=$ 2.0, $\left.1.7 \mathrm{~Hz},{ }_{1 \mathrm{H}}\right), 7.76\left(\mathrm{ddd}, J=7.8,1.7,1.0 \mathrm{~Hz},{ }_{1} \mathrm{H}\right), 7.71(\mathrm{ddd}, J=$ 8.o, 2.0, 1.o Hz, $1 \mathrm{H}), 7.41(\mathrm{dd}, J=8.0,7.8 \mathrm{~Hz}, 1 \mathrm{H}), 3.28-3.22(\mathrm{~m}$, $\left.{ }_{4} \mathrm{H}\right), 1.82-1.76(\mathrm{~m}, 4 \mathrm{H}) .{ }^{13} \mathrm{C}$ NMR: $\left(125 \mathrm{MHz} \mathrm{CDCl}_{3}\right) \delta(\mathrm{ppm})$ $=139.2,135.7,130.7,130.4,126.1,123.2,48.1,25.4$. HRMS: (ESI) $\mathrm{m} / \mathrm{z}$ calculated for $\mathrm{C}_{10} \mathrm{H}_{12} \mathrm{BrNO}_{2} \mathrm{~S}\left[\mathrm{M}+\mathrm{NH}_{4}\right]^{+}$307.0110, found 307.0112.

1-((3-(4,4,5,5-tetramethyl-1,3,2-dioxaborolan-2-yl)phenyl)sulfonyl)pyrrolidine (36). The title compound was prepared according to general procedure $\mathrm{D}$, using aryl-sulfonamide 35 (686 mg, $2.4 \mathrm{mmol}), \mathrm{B}_{2} \mathrm{pin}_{2}$ (66o mg, $2.60 \mathrm{mmol}$ ), $\mathrm{NaOAc}$ (485 mg, $5.9 \mathrm{mmol}$ ) and $\mathrm{Pd}(\mathrm{ddpf}) \mathrm{Cl}_{2}$ (97 mg, 0.12 $\mathrm{mmol})$ in degassed DMSO $(12 \mathrm{~mL})$. Purification of the crude material by column chromatography (EtOAc:hexanes 16:84) afforded 36 as a colorless solid (493 mg, 65\%). ${ }^{\mathbf{H}}$ NMR: (500 $\left.\mathrm{MHz} \mathrm{CDCl}_{3}\right) \delta(\mathrm{ppm})=8.25(\mathrm{dd}, J=2.0,1.4 \mathrm{~Hz}, 1 \mathrm{H}), 7.99(\mathrm{ddd}$, $J=7.4,1.4,1.3 \mathrm{~Hz}, 1 \mathrm{H}), 7.90(\mathrm{ddd}, J=7.9,2.0,1.3 \mathrm{~Hz}, 1 \mathrm{H}), 7.51$ $(\mathrm{dd}, J=7.9,7.4 \mathrm{~Hz}, 1 \mathrm{H}), 3.29-3.22\left(\mathrm{~m},{ }_{4} \mathrm{H}\right), 1.78-1.7 \mathrm{O}\left(\mathrm{m},{ }_{4} \mathrm{H}\right)$, 1.34 (s, 12H). ${ }^{13} \mathrm{C}$ NMR: $\left(125 \mathrm{MHz} \mathrm{CDCl}_{3}\right) \delta(\mathrm{ppm})=138.8,136.6$, 133.7, 130.1, 128.4, 84.5, 48.1, 25.3, 25.0. * ${ }_{13} \mathrm{C}-\mathrm{B}(\mathrm{OR}) 2$ not observed. HRMS: (ESI) $\mathrm{m} / \mathrm{z}$ calculated for $\mathrm{C}_{16} \mathrm{H}_{24} \mathrm{BNO}_{4} \mathrm{~S}[\mathrm{M}+\mathrm{H}]^{+}$ 338.1529 , found 338.1608 .

3-(3-(pyrrolidin-1-ylsulfonyl)phenyl)-1H-indol-5-amine (37). The title compound was prepared according to general procedure A using 7 (100 $\mathrm{mg}, 0.24 \mathrm{mmol}), 3$-pinacolboronate aryl-sulfonamide 36 (82 $\mathrm{mg}, 0.24 \mathrm{mmol}), \mathrm{K}_{2} \mathrm{CO}_{3}$ (101 $\mathrm{mg}, 0.75$ $\mathrm{mmol}), \mathrm{Pd}\left(\mathrm{PPh}_{3}\right)_{4}(28 \mathrm{mg}, 0.024 \mathrm{mmol})$, degassed THF (2.07 $\mathrm{mL})$ and degassed water $(0.7 \mathrm{~mL})$. Purification by column chromatography afforded the protected coupled product, which was subsequently deprotected in TFA (2.4 mL) and concentrated under reduced pressure to afford the TFA salt of 37 as a brown solid (40 mg, 36\%). ${ }^{1} \mathbf{H}$ NMR: (500 $\mathrm{MHz}, \mathrm{CD}_{3} \mathrm{OD}$ ) $\delta(\mathrm{ppm})=8.09(\mathrm{~s}, 1 \mathrm{H}), 7.95(\mathrm{~d}, J=7.6 \mathrm{~Hz}, 1 \mathrm{H}), 7.84(\mathrm{~s}, 1 \mathrm{H}), 7.79$ $(\mathrm{s}, 1 \mathrm{H}), 7.74(\mathrm{~d}, J=7.8 \mathrm{~Hz}, 1 \mathrm{H}), 7.69(\mathrm{dd}, J=7.8,7.6 \mathrm{~Hz}, 1 \mathrm{H})$, $7.61(\mathrm{~d}, J=8.6 \mathrm{~Hz}, 1 \mathrm{H}), 7.18(\mathrm{dd}, J=8.6,2.0 \mathrm{~Hz}, 1 \mathrm{H}), 3.32-3.28$ (s, $4 \mathrm{H}), 1.80-1.76(\mathrm{~m}, 4 \mathrm{H})$. ${ }^{13 C}$ NMR: (125 MHz, CD $\left.3 \mathrm{OD}\right) \delta$ $(\mathrm{ppm})=138.7,138.1,137.9,132.3,131.0,127.3,126.8,126.4,125.7$, $125.6,117.3,116.8,114.5,113.5,49.3,26.3$. HRMS: (ESI) $\mathrm{m} / \mathrm{z}$ calculated for $\mathrm{C}_{18} \mathrm{H}_{19} \mathrm{~N}_{3} \mathrm{O}_{2} \mathrm{~S}[\mathrm{M}+\mathrm{H}]^{+}$342.1271, found 342.1276.

(S)-2-amino-N-(3-(3-(pyrrolidin-1-ylsulfonyl)phenyl)-1Hindol-5-yl)propanamide (15). The title compound was prepared according to general procedure B using the aryl-indole 37 (27 mg, $0.06 \mathrm{mmol})$, (tert-butoxycarbonyl)-L-alanine (11 $\mathrm{mg}, 0.06 \mathrm{mmol}$ ), PyBOP (31 mg, $0.06 \mathrm{mmol}$ ), DIPEA (o.08 mL, $0.30 \mathrm{mmol}$ ), and dry DMF (o.6 mL). RP-HPLC (gradient: 2-50 shortprep, $t R=8.99 \mathrm{~min}$ ) of the crude deprotected product afforded the TFA salt of $\mathbf{1 5}$ as a colorless solid (11 mg, 34\%). ${ }^{\mathbf{1}} \mathbf{H}$ NMR: (50o MHz, CD $30 D) \delta(p p m)=8.29(\mathrm{~s}, 1 \mathrm{H}), 8.10\left(\mathrm{~s},{ }_{1} \mathrm{H}\right)$, $7.94(\mathrm{~d}, J=7.6 \mathrm{~Hz}, 1 \mathrm{H}), 7.68(\mathrm{~d}, J=7.7 \mathrm{~Hz}, 1 \mathrm{H}), 7.644(\mathrm{~s}, 1 \mathrm{H})$, $7.636\left(\mathrm{dd}, J=7.7,7.6 \mathrm{~Hz},{ }_{1} \mathrm{H}\right), 7.45(\mathrm{~d}, J=8.7 \mathrm{~Hz}, 1 \mathrm{H}), 7.27(\mathrm{dd}$, $J=8.7 \mathrm{~Hz}, 1 \mathrm{H}), 4.09(\mathrm{q}, J=7.0 \mathrm{~Hz}, 1 \mathrm{H}), 3.34-3.30(\mathrm{~m}, 4 \mathrm{H}), 1.79$ - $1.75(\mathrm{~m}, 4 \mathrm{H}), 1.63(\mathrm{~d}, J=7.0 \mathrm{~Hz}, 3 \mathrm{H}) .{ }^{13 C}$ NMR: $(125 \mathrm{MHz}$, $\left.\mathrm{CD}_{3} \mathrm{OD}\right) \delta(\mathrm{ppm})=169.0,138.8,138.3,136.2,132.1,132.0,130.7$, $126.4,126.3,125.9,125.3,117.3,116.6,113.2,111.9,50.9,49.4,26.2$, 17.8. HRMS: (ESI) $\mathrm{m} / \mathrm{z}$ calculated for $\mathrm{C}_{21} \mathrm{H}_{24} \mathrm{~N}_{4} \mathrm{O}_{3} \mathrm{~S}[\mathrm{M}+\mathrm{H}]^{+}$ 413.1642, found 413.1643 .

1-bromo-3-(isopropylsulfonyl)benzene (38). The title compound was prepared according to general procedure E, using 3-bromothiophenol (o.66 mL, $6.4 \mathrm{mmol}$, 1.0 equiv.), $\mathrm{K}_{2} \mathrm{CO}_{3}$ (1.23 g, 8.89 mmol, 1.4 equiv.), 2-bromopropane (o.72 mL, 7.6 mmol, 1.2 equiv.) in dry acetone $(21 \mathrm{~mL}, 0.3 \mathrm{M})$. Oxidation was then performed according to general procedure $\mathrm{E}$ using 
bioRxiv preprint doi: https://doi.org/10.1101/2020.11.17.387233; this version posted November 18, 2020. The copyright holder for this preprint (which was not certified by peer review) is the author/funder, who has granted bioRxiv a license to display the preprint in perpetuity. It is made available under aCC-BY-NC-ND 4.0 International license.

$\mathrm{MeOH}$ (36 mL, o.17 M according to the sulfide), oxone (5.593 g, $18 \mathrm{mmol}$, 3.0 equiv.), and water ( $36 \mathrm{~mL}$, $0.5 \mathrm{M}$ according to the oxone). Work-up and concentration of the crude reaction mixture afforded 38 as a yellow oil (1.10 g, $69 \%$ over 2 steps). 'H NMR: (500 $\left.\mathrm{MHz}, \mathrm{CDCl}_{3}\right) \delta(\mathrm{ppm})=8.00(\mathrm{dd}, J=1.9,1.7 \mathrm{~Hz}$, ${ }_{1 \mathrm{H}}$ ), 7.79 (ddd, $J=7.9,1.7,1.1 \mathrm{~Hz}, 1 \mathrm{H}$ ), 7.76 (ddd, $J=8.0$, 2.0, 1.o $\mathrm{Hz}, 1 \mathrm{H}$ ), $7.43(\mathrm{dd}, J=8.0,7.9 \mathrm{~Hz}, 1 \mathrm{H}$ ), 3.19 (septet, $J=6.9 \mathrm{~Hz}$, ${ }_{1 H}$ ), 1.28 (d, $\left.J=6.9 \mathrm{~Hz}, 6 \mathrm{H}\right) .{ }^{13} \mathrm{C}$ NMR: $\left(125 \mathrm{MHz}, \mathrm{CDCl}_{3}\right) \delta$ $(\mathrm{ppm})=139.1,136.8,131.9,130.7,127.7,123.2,55.8,15.7$.

2-(3-(isopropylsulfonyl)phenyl)-4,4,5,5-tetramethyl-1,3,2dioxaborolane (39). The title compound was prepared according to general procedure $\mathrm{D}$, using isopropyl sulfone 38 (1.03 g, $3.92 \mathrm{mmol}), \mathrm{B}_{2} \mathrm{pin}_{2}$ (1.10 g, $\left.4.32 \mathrm{mmol}\right)$, NaOAc (o.805 g, $9.81 \mathrm{mmol})$ and $\mathrm{Pd}(\mathrm{ddpf}) \mathrm{Cl}_{2}(0.160 \mathrm{~g}, 0.20 \mathrm{mmol})$ in degassed DMSO (20 $\mathrm{mL})$. Purification of the crude material by column chromatography (EtOAc:hexanes 1:4) afforded the 3pinacolboronate aryl-sulfone 39 as a colorless solid (o.805 g, $66 \%)$. 'H NMR: $\left(500 \mathrm{MHz}, \mathrm{CDCl}_{3}\right) \delta(\mathrm{ppm})=8.30(\mathrm{dd}, J=\mathbf{2 . 0}$, $1.3 \mathrm{~Hz}, 1 \mathrm{H}$ ), 8.05 (ddd, $J=7.3,1.3,1.3 \mathrm{~Hz}, 1 \mathrm{H}$ ), 7.95 (ddd, $J=7.9$, 2.0, $1.3 \mathrm{~Hz}, 1 \mathrm{H}$ ), $7.55(\mathrm{dd}, J=7.9,7.3 \mathrm{~Hz}, 1 \mathrm{H}$ ), 3.21 (septet, $J=6.9$ $\mathrm{Hz}, 1 \mathrm{H}), 1.34(\mathrm{~s}, 12 \mathrm{H}), 1.29(\mathrm{~d}, J=6.9 \mathrm{~Hz}, 6 \mathrm{H})$. ${ }^{13 C}$ NMR: $(125$ $\left.\mathrm{MHz} \mathrm{CDCl}_{3}\right) \delta(\mathrm{ppm})=139.8,136.8,135.1,131.6,128.4,84.6$, 55.5, 25.0, 15.8. * ${ }_{13} \mathrm{C}-\mathrm{B}(\mathrm{OR}) 2$ not observed. HRMS: (ESI) $\mathrm{m} / \mathrm{z}$ calculated for $\mathrm{C}_{15} \mathrm{H}_{23} \mathrm{BO}_{4} \mathrm{~S}\left[\mathrm{M}+\mathrm{NH}_{4}\right]^{+} 328.1748$, found 328.1760 .

3-(3-(isopropylsulfonyl)phenyl)-1H-indol-5-amine (40). The title compound was prepared according to general procedure A using 7 (597 mg, $1.45 \mathrm{mmol}$ ), isopropyl sulfone 39 (450 $\mathrm{mg}, 1.45 \mathrm{mmol}), \mathrm{K}_{2} \mathrm{CO}_{3}$ (6o2 mg, $\left.4.35 \mathrm{mmol}\right), \mathrm{Pd}\left(\mathrm{PPh}_{3}\right)_{4}(168$ $\mathrm{mg}$, $0.145 \mathrm{mmol})$, degassed THF (12 mL) and degassed water (4 mL). Purification by column chromatography afforded the protected coupled product, which was subsequently deprotected in TFA ( $15 \mathrm{~mL}$ ) and concentrated under reduced pressure to afford the TFA salt of 40 as a brown solid (479 mg,

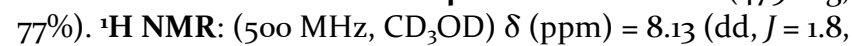
$1.8 \mathrm{~Hz}, 1 \mathrm{H}), 8.02(\mathrm{ddd}, J=7.7,1.8,1.4 \mathrm{~Hz}, 1 \mathrm{H}), 7.89(\mathrm{~d}, J=2.1 \mathrm{~Hz}$, $1 \mathrm{H}), 7.8 \mathrm{o}(\mathrm{s}, 1 \mathrm{H}), 7.78(\mathrm{ddd}, J=7.9,1.8,1.4 \mathrm{~Hz}, 1 \mathrm{H}), 7.71(\mathrm{dd}, J=$ 7.9, $7.7 \mathrm{~Hz}, 1 \mathrm{H}), 7.63(\mathrm{~d}, J=8.6 \mathrm{~Hz}, 1 \mathrm{H}), 7.22(\mathrm{dd}, J=8.6,2.1 \mathrm{~Hz}$, ${ }_{1 \mathrm{H}}$ ), 3.40 (septet, $\left.J=6.8 \mathrm{~Hz},{ }_{1 \mathrm{H}}\right), 1.30(\mathrm{~d}, J=6.8 \mathrm{~Hz}, 6 \mathrm{H}) .{ }^{13} \mathrm{C}$ NMR: (125 MHz, CD $\mathrm{OD}) \delta(\mathrm{ppm})=139.0,138.18,138.16,133.2$, 131.0, 127.7, 127.5, 127.1, 126.7, 124.6, 117.5, 116.6, 114.6, 114.1, 56.5, 15.9. HRMS: (ESI) $\mathrm{m} / \mathrm{z}$ calculated for $\mathrm{C}_{17} \mathrm{H}_{18} \mathrm{~N}_{2} \mathrm{O}_{2} \mathrm{~S}[\mathrm{M}+\mathrm{H}]^{+}$ 315.1162, found 315.1170.

(S)-2-amino-N-(3-(3-(isopropylsulfonyl)phenyl)-1H-indol-5-yl)propanamide (16). The title compound was prepared according to general procedure $B$ using the aryl-indole 40 (56 mg, o.14 mmol), (tert-butoxycarbonyl)- $L$-alanine (30 $\mathrm{mg}$, $0.16 \mathrm{mmol})$, PyBOP (85 mg, $0.16 \mathrm{mmol})$, DIPEA (0.18 mL, $0.68 \mathrm{mmol})$, and dry DMF (1.4 mL). Purification by RP-HPLC (using a SiliCycle SiliaChrom $\mathrm{dtC}_{1} 8$ semipreparative column (5 $\mu \mathrm{m}, 100 \AA$, $10 \times 250 \mathrm{~mm}$ ) with a flow rate of $5 \mathrm{~mL} / \mathrm{min}$ eluting with solvent (A: $0.1 \%$ TFA in water B: $0.1 \%$ TFA in MeCN) on a gradient of $(2 \rightarrow 100) \%$ solvent $B$ over $15 \mathrm{~min}, t R=6.11 \mathrm{~min})$ of the crude deprotected product afforded the TFA salt of $\mathbf{1 6}$ as a colorless solid (10 mg, 15\%). ${ }^{1} \mathbf{H}$ NMR: $\left(600 \mathrm{MHz}, \mathrm{CD}_{3} \mathrm{CN}\right)$ $\delta(\mathrm{ppm})=9.71(\mathrm{~s}, 1 \mathrm{H}), 9.31(\mathrm{~s}, 1 \mathrm{H}), 8.19\left(\mathrm{~d}, J=2.0 \mathrm{~Hz},{ }_{1} \mathrm{H}\right), 8.07$ $(\mathrm{t}, J=1.8 \mathrm{~Hz}, 1 \mathrm{H}), 7.95$ (ddd, $J=7.7,1.5,1.4 \mathrm{~Hz}, 1 \mathrm{H}), 7.71$ (ddd, $J$ $=7.9,1.5,1.4 \mathrm{~Hz}, 1 \mathrm{H}), 7.65(\mathrm{dd}, J=7.9,7.7 \mathrm{~Hz}, 1 \mathrm{H}), 7.63(\mathrm{~d}, J=$ $2.7 \mathrm{~Hz}, 1 \mathrm{H}), 7.44(\mathrm{~d}, J=8.7 \mathrm{~Hz}, 1 \mathrm{H}), 7.33(\mathrm{dd}, J=8.7$, 2.0 Hz, $\left.{ }_{1 \mathrm{H}}\right), 4.24(\mathrm{q}, J=7.1 \mathrm{~Hz}, 1 \mathrm{H}), 3.35$ (septet, $\left.J=6.8 \mathrm{~Hz}, 1 \mathrm{H}\right), 1.60(\mathrm{~d}$, $J=7.1 \mathrm{~Hz}, 3 \mathrm{H}), 1.26(\mathrm{~d}, J=6.8 \mathrm{~Hz}, 6 \mathrm{H}) .13 \mathrm{C} \mathrm{NMR}$ : $(15 \mathrm{O} \mathrm{MHz}$, $\left.\mathrm{CD}_{3} \mathrm{CN}\right) \delta(\mathrm{ppm})=168.5,138.7,137.9,135.2,132.6,132.3,130.6$, 127.4, 126.6, 125.9, 125.8, 116.9, 116.0, 113.2, 110.9, 55.9, 50.9, 17.6, 15.88, 15.87. HRMS: (ESI) $\mathrm{m} / \mathrm{z}$ calculated for $\mathrm{C}_{20} \mathrm{H}_{23} \mathrm{~N}_{3} \mathrm{O}_{3} \mathrm{~S}$ $[\mathrm{M}+\mathrm{H}]+386.1533$, found 386.1538 .

1-bromo-3-(cyclopentylsulfonyl)benzene (41). The title compound was prepared according to general procedure E, using 3-bromothiophenol ( $0.44 \mathrm{~mL}, 4.2 \mathrm{mmol}$, 1.o equiv.), $\mathrm{Cs}_{2} \mathrm{CO}_{3}$ (2.99 g, $8.5 \mathrm{mmol}$, 2.0 equiv.), bromocyclopentane (o.43 mL, 4.2 mmol, 1.o equiv.) in dry DMF (21 mL, o.2 M). Oxidation of the crude sulfide (ca. $713 \mathrm{mg}, 2.8 \mathrm{mmol}$, 1.0 equiv.) was then performed according to general procedure $\mathrm{E}$ using $\mathrm{MeOH}$ (17.0 mL, 0.165 according to the sulfide), and oxone ( $2.56 \mathrm{~g}, 8.3 \mathrm{mmol}$, 3.0 equiv.) in water $(17 \mathrm{~mL}$, 0.5 M). Workup and concentration of the crude reaction mixture afforded the cyclopentyl sulfone 41 as a colorless oil (6oo mg, $49 \%$ over 2 steps). ${ }^{1} \mathrm{H}$ NMR: (50o $\left.\mathrm{MHz}, \mathrm{CDCl}_{3}\right) \delta(\mathrm{ppm})=8.02(\mathrm{dd}, J=$ 2.0, $1.8 \mathrm{~Hz}, 1 \mathrm{H}), 7.81$ (ddd, $J=7.8,1.8,1.1 \mathrm{~Hz}, 1 \mathrm{H}), 7.74(\mathrm{ddd}, J=$ 8.o, 2.0, 1.o Hz, $1 \mathrm{H}), 7.42(\mathrm{dd}, J=8.0,7.8 \mathrm{~Hz}, 1 \mathrm{H}), 3.47(\mathrm{tt}, J=$ 8.7, 7.1 Hz, $1 \mathrm{H}), 2.11-1.96(\mathrm{~m}, 2 \mathrm{H}), 1.92-1.81(\mathrm{~m}, 2 \mathrm{H}), 1.80-$ $1.70(\mathrm{~m}, 2 \mathrm{H}), 1.66-1.53(\mathrm{~m}, 2 \mathrm{H}) .{ }^{13} \mathrm{C}$ NMR: $\left(125 \mathrm{MHz} \mathrm{CDCl}_{3}\right) \delta$ $(\mathrm{ppm})=141.2,136.6,131.4,130.8,127.1,123.3,64.4,27.3,25.9$. HRMS: (ESI) $\mathrm{m} / \mathrm{z}$ calculated for $\mathrm{C}_{11} \mathrm{H}_{13} \mathrm{BrO}_{2} \mathrm{~S}\left[\mathrm{M}+\mathrm{NH}_{4}\right]^{+}$ 306.0158, found 306.0171.

2-(3-(cyclopentylsulfonyl)phenyl)-4,4,5,5-tetramethyl1,3,2-dioxaborolane (42). The title compound was prepared according to general procedure $\mathrm{D}$, using cyclopentyl sulfone 41 (520 mg, $1.8 \mathrm{mmol}$ ), $\mathrm{B}_{2} \mathrm{pin}_{2}$ (502 mg, $1.98 \mathrm{mmol}$ ), NaOAc (369 mg, $4.5 \mathrm{mmol}$ ) and $\mathrm{Pd}(\mathrm{ddpf}) \mathrm{Cl}_{2}$ (73 $\mathrm{mg}$, o.09o mmol) in degassed DMSO (9.0 mL). Purification of the crude material by column chromatography (EtOAc:hexanes 1:4) afforded the 3-pinacolboronate aryl-sulfone 42 as a yellow solid (438 mg, 72 \%). 'H NMR: (500 $\left.\mathrm{MHz}, \mathrm{CDCl}_{3}\right) \delta(\mathrm{ppm})=8.32(\mathrm{dd}, J=\mathbf{2 . 0}, 1.4$ $\mathrm{Hz}, 1 \mathrm{H}$ ), 8.03 (ddd, $J=7.4,1.4,1.2 \mathrm{~Hz}, 1 \mathrm{H}$ ), 7.97 (ddd, $J=7.9$, 2.0, $1.2 \mathrm{~Hz}, 1 \mathrm{H}), 7.54\left(\mathrm{dd}, J=7.9,7.4 \mathrm{~Hz},{ }_{1 \mathrm{H}}\right), 3.51(\mathrm{tt}, J=8.8,7.2$ $\mathrm{Hz}, 1 \mathrm{H}), 2.11-2.03(\mathrm{~m}, 2 \mathrm{H}), 1.90-1.81(\mathrm{~m}, 2 \mathrm{H}), 1.81-1.73(\mathrm{~m}$, $2 \mathrm{H}), 1.65-1.54(\mathrm{~m}, 2 \mathrm{H}), 1.34(\mathrm{~s}, 12 \mathrm{H}) .{ }^{13} \mathrm{C}$ NMR: $(125 \mathrm{MHz}$, $\left.\mathrm{CDCl}_{3}\right) \delta(\mathrm{ppm})=139.7,138.7,134.6,131.0,128.5,84.5,64.2,27.3$, 26.0, 25.o. * ${ }_{13} \mathrm{C}-\mathrm{B}(\mathrm{OR})_{2}$ not observed. HRMS: (ESI) $\mathrm{m} / \mathrm{z}$ calculated for $\mathrm{C}_{17} \mathrm{H}_{25} \mathrm{BO}_{4} \mathrm{~S}\left[\mathrm{M}+\mathrm{NH}_{4}\right]^{+}$354.1905, found 354.1933 .

3-(3-(cyclopentylsulfonyl)phenyl)-1H-indol-5-amine (43). The title compound was prepared according to general procedure A using 7 (200 mg, o.49 mmol), cyclopentyl sulfone 42 (164 mg, $0.49 \mathrm{mmol}), \mathrm{K}_{2} \mathrm{CO}_{3}(202 \mathrm{mg}, 1.46 \mathrm{mmol}), \mathrm{Pd}\left(\mathrm{PPh}_{3}\right)_{4}$ (56 mg, o.049 mmol), degassed THF $(4.14 \mathrm{~mL}$ ) and degassed water $(1.4 \mathrm{~mL})$. Purification by column chromatography afforded the protected coupled product, which was subsequently deprotected in TFA $(4.8 \mathrm{~mL})$ and concentrated under reduced pressure to afford the TFA salt of 43 as a yellow solid (141 mg, 64\%). 'H NMR: (500 MHz, $\left.\mathrm{CD}_{3} \mathrm{OD}\right) \delta(\mathrm{ppm})=8.16(\mathrm{~s}$, $\left.{ }_{1} \mathrm{H}\right), 8.00\left(\mathrm{~d}, J=7.8 \mathrm{~Hz},{ }_{1} \mathrm{H}\right), 7.89\left(\mathrm{~d}, J=2.1 \mathrm{~Hz},{ }_{1} \mathrm{H}\right), 7.90-7.88$ $(\mathrm{m}, 2 \mathrm{H}), 7.71(\mathrm{dd}, J=7.8,7.6 \mathrm{~Hz}, 1 \mathrm{H}), 7.63\left(\mathrm{~d}, J=8.6 \mathrm{~Hz},{ }_{1} \mathrm{H}\right)$, $7.22\left(\mathrm{dd}, J=8.6,2.1 \mathrm{~Hz},{ }_{1} \mathrm{H}\right), 3.76(\mathrm{ddd}, J=15.8,8.9,6.9 \mathrm{~Hz}, 1 \mathrm{H})$, $2.11-1.99(\mathrm{~m}, 2 \mathrm{H}), 1.96-1.86(\mathrm{~m}, 2 \mathrm{H}), 1.81-1.71(\mathrm{~m}, 2 \mathrm{H}), 1.69$ - 1.61 (m, 2H). ${ }^{13} \mathrm{C}$ NMR: (125 MHz, $\left.\mathrm{CD}_{3} \mathrm{OD}\right) \delta(\mathrm{ppm})=140.9$, 138.23, 138.19, 133.1, 131.1, 127.5, 127.1, 126.8, 126.6, 124.6, 117.5, 
bioRxiv preprint doi: https://doi.org/10.1101/2020.11.17.387233; this version posted November 18, 2020. The copyright holder for this preprint (which was not certified by peer review) is the author/funder, who has granted bioRxiv a license to display the preprint in perpetuity. It is made available under aCC-BY-NC-ND 4.0 International license.

116.7, 114.6, 114.1, 65.0, 28.2, 26.9. HRMS: (ESI) $\mathrm{m} / \mathrm{z}$ calculated for $\mathrm{C}_{19} \mathrm{H}_{20} \mathrm{~N}_{2} \mathrm{O}_{2} \mathrm{~S}[\mathrm{M}+\mathrm{H}]^{+}$341.1318, found 341.1318.

\section{Synthesis of sulfone 17}

The title compound was prepared according to general procedure B using the aryl-indole $43(45 \mathrm{mg}$, o.10 mmol), (tertbutoxycarbonyl)- $L$-alanine (19 mg, o.10 mmol), PyBOP (52 mg, o.10 mmol), DIPEA (o.14 mL, o.50 mmol), and dry DMF (1.0 $\mathrm{mL}$ ). RP-HPLC (gradient: 2-50 shortprep, $\mathrm{tR}=7.52 \mathrm{~min}$ ) of the crude deprotected product afforded the TFA salt of 17 as a colorless solid (27 mg, 50\%). ${ }^{1} \mathbf{H}$ NMR: $\left(500 \mathrm{MHz}, \mathrm{CD}_{3} \mathrm{CN}\right) \delta$ $(\mathrm{ppm})=9.74(\mathrm{~s}, 1 \mathrm{H}), 9.18(\mathrm{~s}, 1 \mathrm{H}), 8.17(\mathrm{~d}, J=1.9 \mathrm{~Hz}, 1 \mathrm{H}), 8.09$ (dd, $J=1.8,1.4 \mathrm{~Hz}, 1 \mathrm{H}), 7.93(\mathrm{dd}, J=7.8,1.4,1.4 \mathrm{~Hz}, 1 \mathrm{H}), 7.72$ (dd, $J=7.9,1.4,1.4 \mathrm{~Hz}, 1 \mathrm{H}), 7.64\left(\mathrm{dd}, J=7.9,7.8 \mathrm{~Hz},{ }_{1} \mathrm{H}\right), 7.62$ $(\mathrm{d}, J=2.6 \mathrm{~Hz}, 2 \mathrm{H}), 7.45(\mathrm{~d}, J=8.7 \mathrm{~Hz}, 1 \mathrm{H}), 7.30(\mathrm{dd}, J=8.8,2.4$ $\left.\mathrm{Hz},{ }_{1} \mathrm{H}\right), 4.23(\mathrm{q}, J=7.0 \mathrm{~Hz}, 1 \mathrm{H}), 3.68\left(\mathrm{tt}, J=8.9,7.0 \mathrm{~Hz},{ }_{1} \mathrm{H}\right)$, $2.04-1.96(\mathrm{~m}, 2 \mathrm{H}), 1.91-1.82(\mathrm{~m}, 2 \mathrm{H}), 1.73-1.66(\mathrm{~m}, 2 \mathrm{H}), 1.63$ - $1.55\left(\mathrm{~m},{ }_{5} \mathrm{H}\right) .{ }^{13} \mathrm{C}$ NMR: $\left(125 \mathrm{MHz}, \mathrm{CD}_{3} \mathrm{CN}\right) \delta(\mathrm{ppm})=168.6$, 140.7, 138.1, 135.3, 132.5, 132.2, 130.7, 126.9, 126.1, 126.0, 125.9, 117.1, 116.1, 113.2, 111.2, 64.6, 51.0, 28.0, 27.9, 26.6, 17.6. HRMS: (ESI) $\mathrm{m} / \mathrm{z}$ calculated for $\mathrm{C}_{22} \mathrm{H}_{25} \mathrm{~N}_{3} \mathrm{O}_{3} \mathrm{~S}[\mathrm{M}+\mathrm{H}]^{+}$412.1689, found 412.1706.

(S)-2-amino-N-(3-(3-(cyclopentylsulfonyl)phenyl)-1H-indol-5-yl)propanamide (18a). The title compound was prepared according to general procedure $B$ using the aryl-indole 24 (100 mg, $0.25 \mathrm{mmol}$ ), (tert-butoxycarbonyl)-L-proline (65 mg, $0.30 \mathrm{mmol}$ ), PyBOP (156 mg, 0.30 mmol), DIPEA (o.33 mL, $1.25 \mathrm{mmol}$ ), and dry DMF (2.5 mL). Purification by RP-HPLC (using a SiliCycle SiliaChrom dtC18 semipreparative column (5 $\mu \mathrm{m}, 100 \AA$, $10 \times 250 \mathrm{~mm}$ ) with a flow rate of $5 \mathrm{~mL} / \mathrm{min}$ eluting with solvent (A: $0.1 \%$ TFA in water B: $0.1 \%$ TFA in MeCN) on a gradient of $(2 \rightarrow 100) \%$ solvent $B$ over $15 \mathrm{~min}, t R=5.88 \mathrm{~min})$ of the crude deprotected product afforded the TFA salt of $\mathbf{1 8 a}$ as a pale yellow solid (21 mg, 17\%). ${ }^{\mathbf{1}} \mathbf{H}$ NMR: (6oo $\mathrm{MHz}$, $\left.\mathrm{CD}_{3} \mathrm{OD}\right) \delta(\mathrm{ppm})=8.25(\mathrm{~d}, J=1.9 \mathrm{~Hz}, 1 \mathrm{H}), 8.22(\mathrm{dd}, J=1.9,1.9$ $\left.\mathrm{Hz},{ }_{1} \mathrm{H}\right), 7.99$ (ddd, $\left.J=7.7,1.9,1.8 \mathrm{~Hz}, 1 \mathrm{H}\right), 7.80$ (ddd, $J=7.6,1.9$ $1.8 \mathrm{~Hz}, 1 \mathrm{H}), 7.67(\mathrm{~s}, 1 \mathrm{H}), 7.65(\mathrm{dd}, J=7.7,7.6 \mathrm{~Hz}, 1 \mathrm{H}), 7.45(\mathrm{~d}, J$ $=8.7 \mathrm{~Hz}, 1 \mathrm{H}), 7.34(\mathrm{dd}, J=8.7,1.9 \mathrm{~Hz}, 1 \mathrm{H}), 4.43(\mathrm{dd}, J=7.7,7.7$ $\mathrm{Hz}, 1 \mathrm{H}$ ), 3.48 (ddd, $J=11.4,7.1,7.0 \mathrm{~Hz}, 1 \mathrm{H}), 3.39$ (ddd, $J=11.4$, 71, 7.0 Hz, $1 \mathrm{H}$ ), 3.19 (s, $3 \mathrm{H}$ ), 2.55 (dddd, $J=13.6,7.1,7.0,7.0 \mathrm{~Hz}$, $1 \mathrm{H}), 2.26$ - $2.04(\mathrm{~m}, 3 \mathrm{H}) .{ }_{13 \mathrm{C}} \mathrm{NMR}$ : (150 MHz, CD $\left.3 \mathrm{OD}\right) \delta(\mathrm{ppm})$ $=167.7,142.5$, 139.1, 136.2, 132.9, 132.1, 131.0, 126.3, 126.0, 126.o, 124.9, 117.2, 116.4, 113.2, 111.8, 61.7, 47.5, 44.5, 31.2, 25.2. HRMS: (ESI) $\mathrm{m} / \mathrm{z}$ calculated for $\mathrm{C}_{20} \mathrm{H}_{21} \mathrm{~N}_{3} \mathrm{O}_{3} \mathrm{~S}[\mathrm{M}+\mathrm{H}]^{+} 384.1376$, found 384.1386 .

N1-(3-(3-(methylsulfonyl)phenyl)-1H-indol-5-yl)ethane1,2-diamine (19a). To a stirred solution of the aryl-indole 24 (35 mg, $0.12 \mathrm{mmol}$, 2.o equiv.) in water (ca. $0.15 \mathrm{~mL}$, 2.0 M) was added 2-bromoethan-1-amine hydrochloride (13 mg, 0.061 mmol, 1.o equiv.) at room temperature. The reaction mixture was then stirred at $95^{\circ} \mathrm{C}$ for 22 hours and cooled to room temperature. The reaction mixture was then diluted with water and extracted with EtOAc (3 times). The remaining aqueous layer was purified by RP-HPLC (gradient: 2-30 shortprep, $\mathrm{tR}=$ $8.33 \mathrm{~min}$ ) to afford the TFA salt of 19a as a brown gum (4 $\mathrm{mg}$, 13\%). 'H NMR: (500 MHz, $\left.\mathrm{CD}_{3} \mathrm{CN}\right) \delta(\mathrm{ppm})=9.47\left(\mathrm{~s},{ }_{1} \mathrm{H}\right), 8.17$ $(\mathrm{dd}, J=1.9,1.4 \mathrm{~Hz}, 1 \mathrm{H}), 8.01(\mathrm{ddd}, J=7.8,1.4,1.1 \mathrm{~Hz}, 1 \mathrm{H}), 7.76$ (ddd, $J=7.8,1.9,1.1 \mathrm{~Hz}, 1 \mathrm{H}), 7.67(\mathrm{dd}, J=7.8,7.8 \mathrm{~Hz}, 1 \mathrm{H}), 7.57$ $(\mathrm{d}, J=2.6 \mathrm{~Hz}, 1 \mathrm{H}), 7.36(\mathrm{~d}, J=8.5 \mathrm{~Hz}, 1 \mathrm{H}), 7.16(\mathrm{~s}, 1 \mathrm{H}), 6.75(\mathrm{~d}$, $J=8.5 \mathrm{~Hz}, 1 \mathrm{H}), 3.49(\mathrm{t}, J=5.9 \mathrm{~Hz}, 2 \mathrm{H}), 3.22(\mathrm{t}, J=5.9 \mathrm{~Hz}, 2 \mathrm{H})$, $3.12(\mathrm{~s}, 3 \mathrm{H})$.

N1-methyl-N2-(3-(3-(methylsulfonyl)phenyl)-1H-indol-5yl)ethane-1,2-diamine (19b). To a stirred solution of the arylindole 24 ( $22 \mathrm{mg}$, $0.077 \mathrm{mmol}$, 1.o equiv.) in 1,2-dichloroethane (o.77 mL, o.1 M) was added tert-butyl methyl(2- oxoethyl)carbamate (13 mg, o.077 mmol, 1.o equiv.) at room temperature. The reaction mixture was then stirred at room temperature for 30 minutes, after which $\mathrm{NaBH}(\mathrm{OAc})_{3}$ (24 mg, o.12 mmol, 1.5 equiv.) was added. The solution was then stirred for 18 hours, diluted with saturated aqueous $\mathrm{NaHCO}_{3}$ and extracted with $\mathrm{CH}_{2} \mathrm{Cl}_{2}$ (3 times). The combined organic layers were dried over $\mathrm{MgSO}_{4}$ and concentrated under reduced pressure to afford the crude protected indole (brown gum) which was used in the next step without further purification. The crude protected product was dissolved in TFA (neat, $0.77 \mathrm{~mL}, 0.1 \mathrm{M}$ ) and stirred at room temperature for 30 minutes. The reaction mixture was then concentrated under reduced pressure to afford the crude product, which was purified by RP-HPLC (using a SiliCycle SiliaChrom dtC18 semipreparative column $(5 \mu \mathrm{m}$, $100 \AA$, $10 \times 250 \mathrm{~mm}$ ) with a flow rate of $5 \mathrm{~mL} / \mathrm{min}$ eluting with solvent (A: 0.1 \% TFA in water B: $0.1 \%$ TFA in MeCN) on a gradient of $(2 \rightarrow 30) \%$ solvent $B$ over $15 \mathrm{~min}, t R=8.95 \mathrm{~min})$ to afford the TFA salt of $\mathbf{1 9 b}$ as a brown gum ( $3 \mathrm{mg}, 8 \%$ ). ${ }^{1}$ H NMR: $\left(600 \mathrm{MHz}, \mathrm{CD}_{3} \mathrm{OD}\right) \delta(\mathrm{ppm})=8.26(\mathrm{~s}, 1 \mathrm{H}), 7.99(\mathrm{~d}, J=8.0 \mathrm{~Hz}$, $1 \mathrm{H}), 7.79(\mathrm{~d}, J=7.8 \mathrm{~Hz}, 1 \mathrm{H}), 7.68(\mathrm{dd}, J=7.8,7.8 \mathrm{~Hz}, 1 \mathrm{H}), 7.58$ $(\mathrm{s}, 1 \mathrm{H}), 7.34(\mathrm{~d}, J=8.7 \mathrm{~Hz}, 1 \mathrm{H}), 7.23(\mathrm{~d}, J=2.1 \mathrm{~Hz}, \mathrm{oH}), 6.8 \mathrm{o}(\mathrm{dd}$, $J=8.7,2.1 \mathrm{~Hz}, 1 \mathrm{H}), 3.53(\mathrm{t}, J=6.0 \mathrm{~Hz}, 2 \mathrm{H}), 3.31(\mathrm{t}, J=6.1 \mathrm{~Hz}$, 2H), $3.19\left(\mathrm{~s},{ }_{3} \mathrm{H}\right), 2.78$ (s, $\left.3 \mathrm{H}\right) .{ }^{13} \mathrm{C}$ NMR: (150 MHz, $\left.\mathrm{CD}_{3} \mathrm{OD}\right) \delta$ $(\mathrm{ppm})=143.0,142.4,139.7,133.6,132.6,131.0,127.1,125.8,125.1$, $124.5,115.4,113.9,113.8,102.5,49.6,44.4,42.9,33.7$. HRMS: (ESI) $\mathrm{m} / \mathrm{z}$ calculated for $\mathrm{C}_{18} \mathrm{H}_{21} \mathrm{~N}_{3} \mathrm{O}_{2} \mathrm{~S}[\mathrm{M}+\mathrm{H}]^{+} 344.1427$, found 344.1427 .

(S)-N-(3-(3-(isopropylsulfonyl)phenyl)-1H-indol-5-yl)-2(methylamino)propanamide (18b). The title compound was prepared according to general procedure B using the arylindole 40 (50 mg, $0.12 \mathrm{mmol}$ ), $\mathrm{N}$-(tert-butoxycarbonyl)- $N$-methyl- $L$-alanine (25 mg, $0.12 \mathrm{mmol}$ ), PyBOP (63 mg, o.12 mmol), DIPEA (0.16 mL, o.61 mmol), and dry DMF (1.22 mL). RPHPLC (gradient: $2-30$ shortprep, $t R=11.84 \mathrm{~min}$ ) of the crude deprotected product afforded the TFA salt of $\mathbf{1 8 b}$ as a colorless solid (19 mg, 30\%). 'H NMR: (6oo MHz, $\left.\mathrm{CD}_{3} \mathrm{CN}\right) \delta(\mathrm{ppm})=$ $9.86(\mathrm{~s}, 1 \mathrm{H}), 9.39(\mathrm{~s}, 1 \mathrm{H}), 8.19(\mathrm{~s}, 1 \mathrm{H}), 8.07\left(\mathrm{~d}, J=1.8 \mathrm{~Hz},{ }_{1} \mathrm{H}\right)$, $7.95(\mathrm{dd}, J=7.6,1.7 \mathrm{~Hz}, 1 \mathrm{H}), 7.70(\mathrm{dd}, J=7.8,1.7 \mathrm{~Hz}, 1 \mathrm{H}), 7.65$ $(\mathrm{dd}, J=7.8,7.6 \mathrm{~Hz}, 1 \mathrm{H}), 7.62\left(\mathrm{~d}, J=2.5 \mathrm{~Hz},{ }_{1} \mathrm{H}\right), 7.46(\mathrm{~d}, J=8.6$ $\mathrm{Hz}, 1 \mathrm{H}), 7.32(\mathrm{~d}, J=8.7 \mathrm{~Hz}, 1 \mathrm{H}), 4.06(\mathrm{q}, J=7.0 \mathrm{~Hz}, 1 \mathrm{H}), 3.33$ (septet, $J=6.7 \mathrm{~Hz}, 1 \mathrm{H}), 2.68(\mathrm{~s}, 3 \mathrm{H}), 1.59\left(\mathrm{~d}, J=6.8 \mathrm{~Hz},{ }_{3} \mathrm{H}\right), 1.25$ $(\mathrm{d}, J=6.8 \mathrm{~Hz}, 6 \mathrm{H})$. ${ }^{13 \mathrm{C}} \mathrm{NMR}:\left(150 \mathrm{MHz}, \mathrm{CD}_{3} \mathrm{CN}\right) \delta(\mathrm{ppm})=$ $167.8,138.7,137.9,135.3,132.7,132.0,130.6,127.4,126.6,126.0$, 125.8, 117.1, 116.o, 113.2, 111.3, 58.8, 56.0, 32.1, 16.3, 15.91, 15.90. HRMS: (ESI) $m / z$ calculated for $\mathrm{C}_{21} \mathrm{H}_{25} \mathrm{~N}_{3} \mathrm{O}_{3} \mathrm{~S}[\mathrm{M}+\mathrm{H}]^{+}$ 400.1689, found 400.1703 .

(S)-N-(3-(3-(isopropylsulfonyl)phenyl)-1H-indol-5yl)azetidine-2-carboxamide (18c). The title compound was 
bioRxiv preprint doi: https://doi.org/10.1101/2020.11.17.387233; this version posted November 18, 2020. The copyright holder for this preprint (which was not certified by peer review) is the author/funder, who has granted bioRxiv a license to display the preprint in perpetuity. It is made available under aCC-BY-NC-ND 4.0 International license.

prepared according to general procedure B using the aryl-indole 40 (50 mg, $0.122 \mathrm{mmol}$ ), $N$-Boc- $L$-azetidine-2-carboxylic acid (25 mg, $0.122 \mathrm{mmol}$ ), PyBOP (63 mg, $0.122 \mathrm{mmol}$ ), DIPEA (0.16 mL, o.61 mmol), and dry DMF (1.22 mL). Purification by RP-HPLC (using a SiliCycle SiliaChrom dtC18 semipreparative column $(5 \mu \mathrm{m}, 100 \AA$, $10 \times 250 \mathrm{~mm})$ with a flow rate of $5 \mathrm{~mL} / \mathrm{min}$ eluting with solvent (A: 0.1 \% TFA in water B: $0.1 \%$ TFA in $\mathrm{MeCN})$ on a gradient of $(2 \rightarrow 30) \%$ solvent $B$ over $15 \mathrm{~min}, \mathrm{tR}$ $=11.69 \mathrm{~min}$ ) of the crude deprotected product afforded the TFA salt of $18 \mathrm{c}$ as a colorless solid (14 mg, 23\%). ${ }^{1} \mathbf{H}$ NMR: (6oo $\left.\mathrm{MHz}, \mathrm{CD}_{3} \mathrm{CN}\right) \delta(\mathrm{ppm})=9.81(\mathrm{~s}, 1 \mathrm{H}), 9.36(\mathrm{~s}, 1 \mathrm{H}), 8.21(\mathrm{~s}, 1 \mathrm{H})$, $8.08(\mathrm{~s}, 1 \mathrm{H}), 7.96(\mathrm{~d}, J=7.5 \mathrm{~Hz}, 1 \mathrm{H}), 7.72(\mathrm{~d}, J=7.7 \mathrm{~Hz}, 1 \mathrm{H}), 7.66$ $(\mathrm{dd}, J=7.7,7.5 \mathrm{~Hz}, 1 \mathrm{H}), 7.63(\mathrm{~s}, 1 \mathrm{H}), 7.47\left(\mathrm{~d}, J=8.6 \mathrm{~Hz},{ }_{1} \mathrm{H}\right)$, $7.32(\mathrm{~d}, J=8.7 \mathrm{~Hz}, 1 \mathrm{H}), 5.23(\mathrm{dd}, J=9.4,7.7 \mathrm{~Hz}, 1 \mathrm{H}), 4.16(\mathrm{q}, J=$ $9.3 \mathrm{~Hz}, 1 \mathrm{H}$ ), $3.96(\mathrm{td}, J=10.1,6.4 \mathrm{~Hz}, 1 \mathrm{H}), 3.35$ (septet, $J=6.4$ $\mathrm{Hz}, 1 \mathrm{H}), 2.82(\mathrm{qd}, J=10.1,6.5 \mathrm{~Hz}, 1 \mathrm{H}), 2.64(\mathrm{dt}, J=18.6,8.4 \mathrm{~Hz}$, $1 \mathrm{H}), 1.27(\mathrm{~d}, J=6.5 \mathrm{~Hz}, 6 \mathrm{H}) .{ }^{13} \mathrm{C}$ NMR: $\left(150 \mathrm{MHz}, \mathrm{CD}_{3} \mathrm{CN}\right) \delta$ $(\mathrm{ppm})=166.4,138.8,138.0,135.3,132.7,132.2,130.6,127.4,126.6$, 126.0, 125.9, 116.9, 116.1, 113.3, 111.0, 59.6, 56.0, 44.7, 24.0, 15.9. HRMS: (ESI) $m / z$ calculated for $\mathrm{C}_{21} \mathrm{H}_{23} \mathrm{~N}_{3} \mathrm{O}_{3} \mathrm{~S}[\mathrm{M}+\mathrm{H}]+{ }^{+} 398.1533$, found 398.1564 .

3-(5-amino-1H-indol-3-yl)-N,N-dimethylbenzenesulfonamide (44). The title compound was prepared according to general procedure A using 7 (400 $\mathrm{mg}, 0.97 \mathrm{mmol}$ ), N,N-dimethyl-3-(4,4,5,5-tetramethyl- 1,3,2-dioxaborolan-2-yl)benzenesulfonamide (303 mg, $0.97 \mathrm{mmol}), \mathrm{K}_{2} \mathrm{CO}_{3}(403 \mathrm{mg}, 2.9 \mathrm{mmol}$ ), $\mathrm{Pd}\left(\mathrm{PPh}_{3}\right)_{4}$ (112 mg, o.097 mmol), degassed THF (8.3 mL) and degassed water $(2.8 \mathrm{~mL})$. Purification by column chromatography afforded the protected coupled product, which was subsequently deprotected in TFA (10 mL) and concentrated under reduced pressure to afford the TFA salt of $\mathbf{4 4}$ as a brown solid (261 mg, 62\%). ' $\mathbf{H}$ NMR: (500 MHz, CD $3 \mathrm{OD}) \delta(\mathrm{ppm})=8.03$ $(\mathrm{s}, 1 \mathrm{H}), 7.96(\mathrm{ddd}, J=7.2,1.81 .7 \mathrm{~Hz}, 1 \mathrm{H}), 7.88(\mathrm{~d}, J=2.1 \mathrm{~Hz}, 1 \mathrm{H})$, $7.79(\mathrm{~d}, J=1.5 \mathrm{~Hz}, 1 \mathrm{H}), 7.72-7.66(\mathrm{~m}, 2 \mathrm{H}), 7.63(\mathrm{~d}, J=8.6 \mathrm{~Hz}$, $1 \mathrm{H}), 7.22(\mathrm{dd}, J=8.7,2.1 \mathrm{~Hz}, 1 \mathrm{H}), 2.75(\mathrm{~s}, 6 \mathrm{H})$. 13C NMR: $(125$ $\left.\mathrm{MHz}, \mathrm{CD}_{3} \mathrm{OD}\right) \delta(\mathrm{ppm})=138.2,138.0,137.2,132.4,130.9,127.4$, 126.8, 126.7, 126.0, 124.5, 117.5, 116.8, 114.6, 114.0, 38.4. HRMS: (ESI) $\mathrm{m} / \mathrm{z}$ calculated for $\mathrm{C}_{16} \mathrm{H}_{17} \mathrm{~N}_{3} \mathrm{O}_{2} \mathrm{~S}[\mathrm{M}+\mathrm{H}]^{+} 316.1114$, found 316.1120 .

(S)-N-(3-(3-(N,N-dimethylsulfamoyl)phenyl)-1H-indol-5yl)azetidine-2-carboxamide (18d). The title compound was prepared according to general procedure B using the aryl indole 44 (58mg, o.14mmol), $N$-Boc- $L$-azetidine-2-carboxylic acid (27mg, 0.14mmol), PyBOP (7omg, 0.14mmol), DIPEA (o.179mL, o.68mmol) and dry DMF (1.45mL). Purification by RP-HPLC (using a SiliCycle SiliaChrom dtC18 semipreparative column $(5 \mu \mathrm{m}, 100 \AA$, $10 \times 250 \mathrm{~mm})$ with a flow rate of $5 \mathrm{~mL} / \mathrm{min}$ eluting with solvent (A: $0.1 \%$ TFA in water $B$ : $0.1 \%$ TFA in $\mathrm{MeCN})$ on a gradient of $(2 \rightarrow 50) \%$ solvent $B$ over $15 \mathrm{~min}, \mathrm{tR}$ $=8.60 \mathrm{~min}$ ) of the crude deprotected product afforded the TFA salt of $18 \mathrm{~d}$ as a colorless solid (14 mg, 23\%) (14 mg, 20\%). 'H NMR: (40oMHz, CD $3 \mathrm{CN}) \delta(\mathrm{ppm})=\delta 9.77\left(\mathrm{~s},{ }_{1} \mathrm{H}\right), 9.06(\mathrm{~s}$, $\left.{ }_{1 \mathrm{H}}\right), 8.27(\mathrm{~d}, J=1.8 \mathrm{~Hz}, 1 \mathrm{H}), 8.01(\mathrm{t}, J=1.7,1 \mathrm{H}), 7.96-7.86(\mathrm{~m}$, $1 \mathrm{H}), 7.7 \mathrm{O}-7.59\left(\mathrm{~m},{ }_{3} \mathrm{H}\right), 7.44(\mathrm{~d}, J=8.7 \mathrm{~Hz}, 1 \mathrm{H}), 7.26(\mathrm{dd}, J=$ 8.7, 2.0 Hz, $1 \mathrm{H}), 5.16(\mathrm{t}, J=8.6 \mathrm{~Hz}, 1 \mathrm{H}), 4.31-3.82(\mathrm{~m}, 2 \mathrm{H}), 2.91$ - $2.76(\mathrm{~m}, 1 \mathrm{H}), 2.74(\mathrm{~s}, 6 \mathrm{H}), 2.72-2.55(\mathrm{~m}, 1 \mathrm{H}) .{ }^{13 \mathrm{C}}$ NMR: $(15 \mathrm{O}$ $\left.\mathrm{MHz}, \mathrm{CD}_{3} \mathrm{CN}\right) \delta(\mathrm{ppm})=137.9,136.6,135.2,132.3,132.0,130.7$, 126.4, 126.0, 125.7, 116.7, 113.3, 110.7, 59.8, 44.7, 41.3, 38.7, 24.6.
${ }^{*}{ }_{13} \mathrm{C}=\mathrm{O}$ not observed. HRMS: (ESI) $\mathrm{m} / \mathrm{z}$ calculated for $\mathrm{C}_{20} \mathrm{H}_{23} \mathrm{~N}_{4} \mathrm{O}_{3} \mathrm{~S}[\mathrm{M}+\mathrm{H}]^{+}$399.1485, found 399.1490.

(S)-N-(3-(3-(N,N-dimethylsulfamoyl)phenyl)-1H-indol-5yl)-2-(methylamino)propanamide (18e). The title compound was prepared according to general procedure B using the aryl-indole 44 (50 $\mathrm{mg}, 0.116 \mathrm{mmol}), \mathrm{N}$-(tert-butoxycarbonyl)- $N$-methyl- $L$-alanine (24 mg, $0.116 \mathrm{mmol}$ ), PyBOP (61 $\mathrm{mg}$, $0.116 \mathrm{mmol}$ ), DIPEA (o.15 mL, $0.58 \mathrm{mmol})$, and dry DMF (1.2 mL). Purification by RP-HPLC (using a SiliCycle SiliaChrom dtC18 semipreparative column $(5 \mu \mathrm{m}, 100 \AA$, $10 \times 250$ $\mathrm{mm}$ ) with a flow rate of $5 \mathrm{~mL} / \mathrm{min}$ eluting with solvent (A: 0.1 $\%$ TFA in water $\mathrm{B}$ : $0.1 \%$ TFA in $\mathrm{MeCN})$ on a gradient of $(2 \rightarrow$ 30) \% solvent $B$ over $15 \mathrm{~min}, \mathrm{tR}=11.84 \mathrm{~min}$ ) of the crude deprotected product afforded the TFA salt of $\mathbf{1 8 e}$ as a colorless solid (20 mg, 34\%). 'H NMR: (500 MHz, $\left.\mathrm{CD}_{3} \mathrm{OD}\right) \delta(\mathrm{ppm})=8.31(\mathrm{~d}$, $J=2.0 \mathrm{~Hz}, 1 \mathrm{H}), 8.06(\mathrm{dd}, J=1.8 \mathrm{~Hz}, 1 \mathrm{H}), 7.96(\mathrm{ddd}, J=7.2,1.8$, $1.7 \mathrm{~Hz}, 1 \mathrm{H}), 7.7 \mathrm{O}-7.61\left(\mathrm{~m},{ }_{3} \mathrm{H}\right), 7.45(\mathrm{~d}, J=8.7 \mathrm{~Hz}, 1 \mathrm{H}), 7.27(\mathrm{dd}$, $J=8.7,2.0 \mathrm{~Hz}, 1 \mathrm{H}), 3.87(\mathrm{q}, J=7.0 \mathrm{~Hz}, 1 \mathrm{H}), 2.77(\mathrm{~s}, 6 \mathrm{H}), 2.7 \mathrm{O}(\mathrm{s}$, $\left.{ }_{3} \mathrm{H}\right), 1.60(\mathrm{~d}, J=7.0 \mathrm{~Hz}, 3 \mathrm{H}) .{ }^{13 \mathrm{C}}$ NMR: $\left(150 \mathrm{MHz}, \mathrm{CD}_{3} \mathrm{OD}\right) \delta$ $(\mathrm{ppm})=169.14,138.8,136.8,136.2$, 132.2, 132.0, 130.7, 126.6, 126.4, $125.9,125.6,117.1,116.5,113.2,111.8,59.3,38.6,32.2,16.9$. HRMS: (ESI) $\mathrm{m} / \mathrm{z}$ calculated for $\mathrm{C}_{20} \mathrm{H}_{24} \mathrm{~N}_{4} \mathrm{O}_{3} \mathrm{~S}[\mathrm{M}+\mathrm{H}]^{+} 401.1642$, found 401.1655 .

2-amino-N-(3-(3-(N,N-dimethylsulfamoyl)phenyl)-1H-indol-5-yl)acetamide (18f). The title compound was prepared according to general procedure B using the aryl-indole 44 ( 45 $\mathrm{mg}, \quad 0.10 \mathrm{mmol}$ ), (tert-butoxycarbonyl)glycine (18 mg, 0.10 mmol), PyBOP (54 mg, o.10 mmol), DIPEA (o.14 mL, 0.52 mmol), and dry DMF (1.0 mL). RP-HPLC (gradient: 2-50 shortprep, $\mathrm{tR}=10.38 \mathrm{~min}$ ) of the crude deprotected product afforded the TFA salt of $\mathbf{1 8 f}$ as a colorless solid (13 mg, 25\%). 'H NMR: (50o MHz, CD 3 OD: $\mathrm{CD}_{3} \mathrm{CN}$ 1:1, calibrated to $\mathrm{CD}_{3} \mathrm{OD}$ ) $\delta(\mathrm{ppm})=8.32\left(\mathrm{~d}, J=2.0 \mathrm{~Hz},{ }_{1} \mathrm{H}\right), 8.07(\mathrm{~s}, 1 \mathrm{H}), 7.98(\mathrm{~d}, J=7.2$ $\mathrm{Hz}, 1 \mathrm{H}), 7.72-7.65(\mathrm{~m}, 3 \mathrm{H}), 7.50(\mathrm{~d}, J=8.7 \mathrm{~Hz}, 1 \mathrm{H}), 7.29$ (dd, $J$ $\left.=8.7,2.0 \mathrm{~Hz},{ }_{1} \mathrm{H}\right), 3.85(\mathrm{~s}, 2 \mathrm{H}), 2.78(\mathrm{~s}, 6 \mathrm{H}) .{ }^{13} \mathrm{C}$ NMR: $(125 \mathrm{MHz}$, $\mathrm{CD}_{3} \mathrm{OD}: \mathrm{CD}_{3} \mathrm{CN}$ 1:1, calibrated to $\left.\mathrm{CD}_{3} \mathrm{OD}\right) \delta(\mathrm{ppm})=164.9$, 138.3, 136.7, 135.6, 132.13, 132.07, 130.7, 126.4, 126.12, 126.08, 125.6, 116.9, 116.2, 113.3, 111.2, 42.0, 38.6. HRMS: (ESI) m/z calculated for $\mathrm{C}_{18} \mathrm{H}_{20} \mathrm{~N}_{4} \mathrm{O}_{3} \mathrm{~S}[\mathrm{M}+\mathrm{H}]^{+}$373.1329, found 373.1343.

2-amino-N-(3-(3-(N,N-dimethylsulfamoyl)phenyl)-1H-indol-5-yl)-2-methylpropanamide (18g). The title compound was prepared according to general procedure B using the arylindole 44 (50 $\mathrm{mg}, 0.116 \mathrm{mmol})$, N-Boc- $\alpha$-methyl alanine (24 $\mathrm{mg}, 0.116 \mathrm{mmol})$, PyBOP (6o1 mg, $0.116 \mathrm{mmol})$, DIPEA (o.15 $\mathrm{mL}, 0.58 \mathrm{mmol})$, and dry DMF (1.2 mL). RP-HPLC (gradient: 2-50 shortprep, $t R=8.50 \mathrm{~min}$ ) of the crude deprotected product afforded the TFA salt of $\mathbf{1 8 g}$ as a colorless solid (17 $\mathrm{mg}$, 28\%). ' ${ }^{H}$ NMR: ( $\left.500 \mathrm{MHz}, \mathrm{CD}_{3} \mathrm{CN}\right) \delta(\mathrm{ppm})=9.75(\mathrm{~s}, 1 \mathrm{H}), 8.75$ $(\mathrm{s}, 1 \mathrm{H}), 8.20(\mathrm{~d}, J=1.9 \mathrm{~Hz}, 1 \mathrm{H}), 8.01(\mathrm{dd}, J=1.7,1.6 \mathrm{~Hz}, 1 \mathrm{H}), 7.95$ (ddd, $J=7.3,1.7,1.6 \mathrm{~Hz}, 1 \mathrm{H}), 7.71-7.60(\mathrm{~m}, 3 \mathrm{H}), 7.50$ (d, $J=8.7$ $\mathrm{Hz}, 1 \mathrm{H}), 7.34(\mathrm{dd}, J=8.7,1.9 \mathrm{~Hz}, 1 \mathrm{H}), 2.73(\mathrm{~s}, 6 \mathrm{H}), 1.72(\mathrm{~s}, 6 \mathrm{H})$. 13C NMR: (125 MHz, CD 3 CN) $\delta(\mathrm{ppm})=170.6,137.8$, 136.5, 135.4, 131.9, 131.8, 130.6, 126.3, 125.9, 125.8, 125.6, 117.9, 116.2, 113.1, 112.2, 59.2, 38.6, 24.1. HRMS: (ESI) $\mathrm{m} / \mathrm{z}$ calculated for $\mathrm{C}_{20} \mathrm{H}_{24} \mathrm{~N}_{4} \mathrm{O}_{3} \mathrm{~S}$ $[\mathrm{M}+\mathrm{H}]^{+} 401.1642$, found 401.1652 . 
bioRxiv preprint doi: https://doi.org/10.1101/2020.11.17.387233; this version posted November 18, 2020. The copyright holder for this preprint (which was not certified by peer review) is the author/funder, who has granted bioRxiv a license to display the preprint in perpetuity. It is made available under aCC-BY-NC-ND 4.0 International license.

1-amino-N-(3-(3-(N,N-dimethylsulfamoyl)phenyl)-1H-indol-5-yl)cyclopropane-1-carboxamide (18h). The title compound was prepared according to general procedure B using the aryl-indole $44(50 \mathrm{mg}, 0.116 \mathrm{mmol}), \mathrm{r}-(($ tert-butoxycarbonyl)amino)- cyclopropane-1-carboxylic acid (23 mg, 0.116 mmol), РyBOP (61 mg, $0.116 \mathrm{mmol}$ ), DIPEA (o.15 mL, 0.58 mmol), and dry DMF (1.16 mL). RP-HPLC (gradient: 2-50 shortprep, $t R=8.43 \mathrm{~min}$ ) of the crude deprotected product afforded the TFA salt of $\mathbf{1 8 h}$ as a colorless solid (16 mg, $27 \%$ ). 'H NMR: $\left(500 \mathrm{MHz}, \mathrm{CD}_{3} \mathrm{CN}\right) \delta(\mathrm{ppm})=9.68(\mathrm{~s}, 1 \mathrm{H}), 8.12(\mathrm{~d}, J$ $=1.9 \mathrm{~Hz}, 1 \mathrm{H}), 8.01(\mathrm{dd}, J=1.7,1.7 \mathrm{~Hz}, 1 \mathrm{H}), 7.93$ (ddd, $J=7.2,1.8$, $1.7 \mathrm{~Hz}, 1 \mathrm{H}), 7.89(\mathrm{~s}, 1 \mathrm{H}), 7.7 \mathrm{o}-7.62(\mathrm{~m}, 3 \mathrm{H}), 7.49(\mathrm{~d}, J=8.7 \mathrm{~Hz}$, $1 \mathrm{H}), 7.26(\mathrm{dd}, J=8.8,2.0 \mathrm{~Hz}, 1 \mathrm{H}), 2.74(\mathrm{~s}, 6 \mathrm{H}), 1.74-1.68(\mathrm{~m}$, $2 \mathrm{H}), 1.60-1.54(\mathrm{~m}, 2 \mathrm{H})$. ${ }^{13 C}$ NMR: $\left(125 \mathrm{MHz}, \mathrm{CD}_{3} \mathrm{CN}\right) \delta(\mathrm{ppm})$ $=167.1,136.9,135.8,134.6,131.0,130.3,129.7,125.4,125.0,124.9$, $124.7,118.2,115.3,112.2,111.7,37.6,36.6,12.4$. HRMS: (ESI) $\mathrm{m} / \mathrm{z}$ calculated for $\mathrm{C}_{20} \mathrm{H}_{22} \mathrm{~N}_{4} \mathrm{O}_{3} \mathrm{~S}[\mathrm{M}+\mathrm{H}]^{+} 399.1485$, found 399.1495 .

3-(5-(1,3-dioxoisoindolin-2-yl)-1H-indol-3-yl)-N,N-dimethylbenzenesulfonamide (45). The title compound was prepared according to the general procedures I and F using $\mathbf{2 8}$ (64 $\mathrm{mg}$, $0.12 \mathrm{mmol})$, TFA ( $1.2 \mathrm{~mL})$, phthalic anhydride $(18 \mathrm{mg}$, 0.12 $\mathrm{mmol}$ ) and dry toluene $(0.5 \mathrm{~mL})$. Purification by column chromatography (EtOAc:hexanes 10:90) afforded 45 as a pale yellow solid (42 mg, 78\%). ${ }^{1} \mathbf{H}$ NMR: (50o $\left.\mathrm{MHz}, \mathrm{CDCl}_{3}\right) \delta(\mathrm{ppm})$ $=8.58(\mathrm{br} \mathrm{s}, 1 \mathrm{H}), 7.98(\mathrm{~d}, J=3.0 \mathrm{~Hz}, 1 \mathrm{H}), 7.96(\mathrm{~d}, J=3.0 \mathrm{~Hz}, 1 \mathrm{H})$, 7.89 (br d, $J=8.5 \mathrm{~Hz}, 2 \mathrm{H}), 7.8 \mathrm{o}(\mathrm{d}, J=3.0 \mathrm{~Hz}, 1 \mathrm{H}), 7.79(\mathrm{~d}, J=$ 3.o Hz, $1 \mathrm{H}), 7.67(\mathrm{br} d, J=7.7 \mathrm{~Hz}, 1 \mathrm{H}), 7.6 \mathrm{o}-7.55(\mathrm{~m}, 2 \mathrm{H}), 7.49$ (br t, $J=2.2 \mathrm{~Hz}, 1 \mathrm{H}), 7.29($ br d, $J=8.8 \mathrm{~Hz}, 1 \mathrm{H}), 2.75(\mathrm{~s}, 6 \mathrm{H})$.

3-(5-(1,3-dioxoisoindolin-2-yl)-1-methyl-1H-indol-3-yl)$\mathrm{N}, \mathrm{N}$-dimethylbenzenesulfonamide (46). The title compound was prepared according to general procedure $\mathrm{G}$ using 45 (42 mg, o.094 mmol), $\mathrm{K}_{2} \mathrm{CO}_{3}(65 \mathrm{mg}, 0.47 \mathrm{mmol}), \mathrm{MeI}$ (o.018mL, $0.28 \mathrm{mmol}$ ) and dry DMF (0.5 mL). The crude product was directly used in the next step without further purification. ${ }^{1} \mathrm{H}$ NMR: $\left(500 \mathrm{MHz}, \mathrm{CDCl}_{3}\right) \delta(\mathrm{ppm})=7.97(\mathrm{br} \mathrm{s}, 1 \mathrm{H}), 7.97$ $(\mathrm{d}, J=3.12 \mathrm{~Hz}, 1 \mathrm{H}), 7.96(\mathrm{~d}, J=3.0 \mathrm{~Hz}, 1 \mathrm{H}), 7.90(\mathrm{br} \mathrm{d}, J=1.9$ $\mathrm{Hz}, 1 \mathrm{H}), 7.88(\mathrm{ddd}, J=7.7,1.5,1.3 \mathrm{~Hz}, 1 \mathrm{H}), 7.8 \mathrm{o}(\mathrm{d}, J=3.0 \mathrm{~Hz}$, $1 \mathrm{H}), 7.79(\mathrm{~d}, J=3.0 \mathrm{~Hz}, 1 \mathrm{H}), 7.65(\mathrm{ddd}, J=7.9,1.4,1.3 \mathrm{~Hz}, 1 \mathrm{H})$, $7.57(\mathrm{dd}, J=7.9,7.7 \mathrm{~Hz}, 1 \mathrm{H}), 7.5 \mathrm{O}(\mathrm{d}, J=8.7 \mathrm{~Hz}, 1 \mathrm{H}), 7.4 \mathrm{O}(\mathrm{s}$, $1 \mathrm{H}), 7.32(\mathrm{dd}, J=8.7,1.9 \mathrm{~Hz}, 1 \mathrm{H}), 3.91(\mathrm{~s}, 3 \mathrm{H}), 2.75(\mathrm{~s}, 6 \mathrm{H})$.

3-(5-amino-1-methyl-1H-indol-3-yl)-N,N-dimethylbenzenesulfonamide (47). The title compound was prepared according to general procedure $\mathrm{H}$ using 46 (37 mg, o.08o mmol), hydrazine hydrate $(3.4 \mathrm{mg}$, o.10 mmol) and $\mathrm{MeOH}(1.3 \mathrm{~mL})$. The crude product was directly used in the next step without further purification. ' $\mathbf{H}$ NMR: $\left(500 \mathrm{MHz}, \mathrm{CDCl}_{3}\right) \delta(\mathrm{ppm})=$ 7.99 (br t, $J=1.7 \mathrm{~Hz}, 1 \mathrm{H}), 7.85(\mathrm{dt}, J=7.5,1.5 \mathrm{~Hz}, 1 \mathrm{H}), 7.60(\mathrm{dt}$, $J=8.0,1.7 \mathrm{~Hz}, 1 \mathrm{H}), 7.55(\mathrm{dd}, J=17.2,7.8 \mathrm{~Hz}, 1 \mathrm{H}), 7.25(\mathrm{~s}, 1 \mathrm{H})$, $7.21(\mathrm{~d}, J=2.2 \mathrm{~Hz}, 1 \mathrm{H}), 7.19(\mathrm{~d}, J=8.5 \mathrm{~Hz}, 1 \mathrm{H}), 6.77(\mathrm{dd}, J=8.5$, $2.2 \mathrm{~Hz}, 1 \mathrm{H}), 3.8 \mathrm{o}(\mathrm{s}, 3 \mathrm{H}), 2.75(\mathrm{~s}, 3 \mathrm{H})$.

(S)-2-amino- $\mathrm{N}$-(3-(3-(N,N-dimethylsulfamoyl)phenyl)-1methyl-1H-indol-5-yl)propanamide (2o). The title compound was prepared according to general procedure B using the aryl-indole $47(28 \mathrm{mg}, 0.08 \mathrm{o} \mathrm{mmol})$, (tert-butoxycarbonyl)-L-alanine ( $18 \mathrm{mg}, 0.095 \mathrm{mmol})$, PyBOP (50 mg, 0.38 mmol), DIPEA (o.07o mL, $0.095 \mathrm{mmol}$ ), and dry DMF (o.8
$\mathrm{mL}$ ) followed by Boc-deprotection using TFA (o.7 mL). Purification by RP-HPLC (using a SiliCycle SiliaChrom dtC18 semipreparative column $(5 \mu \mathrm{m}, 100 \AA$, $10 \times 250 \mathrm{~mm})$ with a flow rate of $5 \mathrm{~mL} / \mathrm{min}$ eluting with solvent (A: 0.1 \% TFA in water B: 0.1 $\%$ TFA in MeCN) on a gradient of $(2 \rightarrow 100) \%)$ solvent B over $15 \mathrm{~min}, \mathrm{t}_{\mathrm{R}}=6.5 \mathrm{~min}$ ) of the crude deprotected product afforded the TFA salt of 20 as a colorless solid (10 mg, $15 \%)$. ${ }^{1} \mathbf{H}$ NMR: $\left(400 \mathrm{MHz}, \mathrm{CD}_{3} \mathrm{CN}\right) \delta(\mathrm{ppm})=9.23(\mathrm{~s}, 1 \mathrm{H}), 8.23(\mathrm{~d}, J=1.6 \mathrm{~Hz}$, $1 \mathrm{H}), 7.96(\mathrm{br} \mathrm{s}, 1 \mathrm{H}), 7.87(\mathrm{dt}, J=7.4,1.6 \mathrm{~Hz}, 1 \mathrm{H}), 7.63(\mathrm{t}, J=7.4$ $\mathrm{Hz}, 1 \mathrm{H}), 7.6 \mathrm{o}(\mathrm{t}, J=1.6 \mathrm{~Hz}, 1 \mathrm{H}), 7.55(\mathrm{~s}, 1 \mathrm{H}), 7.35(\mathrm{dq}, J=8.9$, o.8 $\left.\mathrm{Hz},{ }_{1} \mathrm{H}\right), 7.29(\mathrm{dd}, J=8.9,1.9 \mathrm{~Hz}, 1 \mathrm{H}), 4.24\left(\mathrm{q}, J=7.0 \mathrm{~Hz},{ }_{1} \mathrm{H}\right)$, $3.79(\mathrm{~s}, 3 \mathrm{H}), 2.71(\mathrm{~s}, 6 \mathrm{H}), 1.58(\mathrm{~d}, J=7.0 \mathrm{~Hz}, 3 \mathrm{H}) .{ }^{13} \mathrm{C}$ NMR: $(15 \mathrm{O}$ $\left.\mathrm{MHz}, \mathrm{CD}_{3} \mathrm{CN}\right) \delta(\mathrm{ppm})=168.6,137.7,136.6,136.0,132.2$, 131.6, 130.6, 130.2, 126.2, 126.1, 125.4, 116.8, 114.9, 111.4, 111.3, 50.96, 38.6o, 33.47, 17.62. HRMS: (ESI) $\mathrm{m} / \mathrm{z}$ calculated for $\mathrm{C}_{21} \mathrm{H}_{24} \mathrm{~N}_{4} \mathrm{O}_{3} \mathrm{~S}[\mathrm{M}+\mathrm{H}]^{+}$401.1647, found 401.1602 .

((benzyloxy)carbonyl)-L-serine (48). $1.17 \mathrm{~g} \mathrm{NaHCO}_{3}(14$ mmol, 1 equiv.) and $3.45 \mathrm{~g} \mathrm{Na}_{2} \mathrm{CO}_{3}(28 \mathrm{mmol}, 2$ equiv.) were dissolved in $3 \mathrm{~mL}$ acetone and $32 \mathrm{~mL} \mathrm{H}_{2} \mathrm{O}$. L-serine $(1.46 \mathrm{~g}$, 14 mmol, 1 equiv.) was then added, followed by $\mathrm{CbzCl}(2.47 \mathrm{~mL}$, $17 \mathrm{mmol}, 1.25$ equiv.). The mixture was then stirred vigorously at room temperature. Each time the mixture became cloudy, $\mathrm{IM} \mathrm{KOH}$ was added until it became a clear solution again. After $5 \mathrm{~h}$, the reaction mixture was washed three times with $\mathrm{Et}_{2} \mathrm{O}$ and the aqueous layer was acidified with concentrated $\mathrm{HCl}$. Filtration afforded the title compound as a white powder (2.38 g, $72 \%) .{ }^{1} \mathrm{H}$ NMR: $\left(400 \mathrm{MHz}, \mathrm{DMSO}-\mathrm{d}_{6}\right) \delta(\mathrm{ppm})=12.63(\mathrm{~s}$, $1 \mathrm{H}), 7.42-7.27(\mathrm{~m}, 5 \mathrm{H}), 5.05(\mathrm{~s}, 2 \mathrm{H}), 4.11-4.02(\mathrm{~m}, 1 \mathrm{H}), 3.72-$ $3.60(\mathrm{~m}, 2 \mathrm{H})$.

$\mathrm{N}$-((benzyloxy)carbonyl)-O-(tert-butyldimethylsilyl)-Lserine (49). To a suspension of 48 (2.38 g, $10 \mathrm{mmol}, 1$ equiv.) and imidazole (2.03 g, $30 \mathrm{mmol}, 3$ equiv.) in $\mathrm{CH}_{2} \mathrm{Cl}_{2}(56 \mathrm{~mL})$ were added TBSCl (1.65 g, $11 \mathrm{mmol}$, 1.1 equiv.) and DMAP (61 $\mathrm{mg}, 0.50 \mathrm{mmol}, 0.05$ equiv.) and the resulting solution was then stirred at room temperature overnight. The reaction mixture was then diluted with $1 \mathrm{M} \mathrm{HCl}$ and $\mathrm{H}_{2} \mathrm{O}$ and the layers were separated. The aqueous were extracted twice more with $\mathrm{CH}_{2} \mathrm{Cl}_{2}$ and the combined organic were washed with $1 \mathrm{M} \mathrm{HCl}$, $\mathrm{H}_{2} \mathrm{O}$ and brine, dried over $\mathrm{Na}_{2} \mathrm{SO}_{4}$, filtered and evaporated to afford a crude oil. This material was redissolved in $\mathrm{MeOH} / \mathrm{PhMe}$ and the solvents were evaporated again. This process of $\mathrm{MeOH} / \mathrm{PhMe}$ addition/evaporation was repeated twice more to afford the title compound as a thick white oil (2.81 g, 8o\%). ${ }^{1} \mathbf{H}$ NMR: (400 MHz, $\left.\mathrm{CDCl}_{3}\right) \delta(\mathrm{ppm})=7.45-$ $7.29(\mathrm{~m}, 5 \mathrm{H}), 5.58(\mathrm{~d}, J=8.2 \mathrm{~Hz}, 1 \mathrm{H}), 5.19-5.05(\mathrm{~m}, 2 \mathrm{H}), 4.44$ $(\mathrm{t}, J=5.8 \mathrm{~Hz}, 1 \mathrm{H}), 4.17-4.05(\mathrm{~m}, \mathrm{lH}), 3.85(\mathrm{td}, J=9.9,3.5 \mathrm{~Hz}$, $1 \mathrm{H}), 0.86(\mathrm{~s}, 9 \mathrm{H}), 0.03(\mathrm{~s}, 6 \mathrm{H})$.

benzyl (S)-4-(((tert-butyldimethylsilyl)oxy)methyl)-5oxooxazolidine-3-carboxylate (50). A flask was charged with 49 (2.81 g, $8 \mathrm{mmol}$, 1 equiv.), pTsOH. $\mathrm{H}_{2} \mathrm{O}(227 \mathrm{mg}, 1.2$ mmol, o.15 equiv.), $\left(\mathrm{CH}_{2} \mathrm{O}\right)_{\mathrm{n}}(1.40 \mathrm{~g})$ and dry $\mathrm{PhMe}(115 \mathrm{~mL})$. A Dean-Stark apparatus was attached and the mixture was heated to reflux for $\mathrm{th}$. The reaction mixture was then washed with saturated $\mathrm{NaHCO}_{3}, \mathrm{H}_{2} \mathrm{O}$ and brine, dried over $\mathrm{Na}_{2} \mathrm{SO}_{4}$, filtered and evaporated to afford $4.34 \mathrm{~g}$ of a crude yellow oil. Purification by flash column chromatography (EtOAc:hexanes 30:70) afforded the title compound as a colourless oil (1.81 g, 62\%). ${ }^{1} \mathrm{H}$ NMR: (400 MHz, $\left.\mathrm{CDCl}_{3}\right) \delta(\mathrm{ppm})=7.43-7.30(\mathrm{~m}$, 
bioRxiv preprint doi: https://doi.org/10.1101/2020.11.17.387233; this version posted November 18, 2020. The copyright holder for this preprint (which was not certified by peer review) is the author/funder, who has granted bioRxiv a license to display the preprint in perpetuity. It is made available under aCC-BY-NC-ND 4.0 International license.

$\left.{ }_{5} \mathrm{H}\right), 5.53(\mathrm{~d}, J=27.9 \mathrm{~Hz}, 1 \mathrm{H}), 5.22(\mathrm{~s}, 2 \mathrm{H}), 4.38-4.16(\mathrm{~m}, 3 \mathrm{H})$, $4.05-3.98(\mathrm{~m}, 1 \mathrm{H}), 0.84(\mathrm{~s}, 9 \mathrm{H}), 0.10\left(\mathrm{~s},{ }_{3} \mathrm{H}\right)$, o.01 (s, $\left.3 \mathrm{H}\right)$.

benzyl (R)-4-(fluoromethyl)-5-oxooxazolidine-3-carboxylate (51). To a solution of $\mathbf{5 0}(\mathbf{1 . 8 1} \mathrm{g}, 5 \mathrm{mmol}, 1$ equiv.) in dry $\mathrm{CH}_{2} \mathrm{Cl}_{2}(25 \mathrm{~mL})$ at $-78^{\circ} \mathrm{C}$ were added $\mathrm{Et}_{3} \mathrm{~N}$.HF $(3.23 \mathrm{~mL}, 20$ mmol, 4 equiv.) and Xtalfluor-E (2.27 g, $10 \mathrm{mmol}, 2$ equiv.). The mixture was stirred for $1 \mathrm{~h}$ at $-78^{\circ} \mathrm{C}$ and then allowed to warm to room temperature overnight. The reaction mixture was then diluted with saturated $\mathrm{NaHCO}_{3}$ and stirred vigorously for 2omin. The layers were then separated and the aqueous was extracted twice more with $\mathrm{CH}_{2} \mathrm{Cl}_{2}$. The combined organic were washed with saturated $\mathrm{NaHCO}_{3}, \mathrm{H}_{2} \mathrm{O}$ and brine, dried over $\mathrm{Na}_{2} \mathrm{SO}_{4}$, filtered and evaporated to afford $847 \mathrm{mg}$ of a brown oil. Purification by flash column chromatography (EtOAc:hexanes 30:70) afforded the title compound as a pale yellow oil (442 mg, 35\%). ${ }^{1}$ H NMR: (400 $\mathrm{MHz} \mathrm{CDCl}_{3}$ ) $\delta$ (ppm) $=7.44-7.32(\mathrm{~m}, 5 \mathrm{H}), 5.55(\mathrm{~s}, 1 \mathrm{H}), 5.36-5.15\left(\mathrm{~m},{ }_{3} \mathrm{H}\right), 4.83(\mathrm{dd}$, $J=45.0,9.3 \mathrm{~Hz}, 2 \mathrm{H}), 4.39(\mathrm{~d}, J=33.9 \mathrm{~Hz}, 1 \mathrm{H}) .19 F \mathbf{N M R}(376$ $\left.\mathrm{MHz}, \mathrm{CDCl}_{3}\right) \delta(\mathrm{ppm})=(-231.56-(-232.71))(\mathrm{m}, \mathrm{l} \mathrm{F})$.

(R)-2-((tert-butoxycarbonyl)amino)-3-fluoropropanoic acid (52). To a solution of $\mathbf{5 1}(241 \mathrm{mg}, 0.95 \mathrm{mmol}, 1$ equiv.) in dry $\mathrm{CH}_{2} \mathrm{Cl}_{2}(8 \mathrm{~mL})$ at $\mathrm{o}{ }^{\circ} \mathrm{C}$ was added $1 \mathrm{M} \mathrm{BCl}_{3}$ in $\mathrm{CH}_{2} \mathrm{Cl}_{2}(3.81$ $\mathrm{mL}, 3.81 \mathrm{mmol}, 4$ equiv.) dropwise. The ice bath was removed and the mixture was allowed to warm to room temperature over $25 \mathrm{~min}$. The reaction mixture was then poured into $25 \mathrm{~mL}$ ice-water and the layers were separated. The aqueous layer was washed once with $\mathrm{CH}_{2} \mathrm{Cl}_{2}$ and then it was neutralized with ${ }_{5} \mathrm{M} \mathrm{KOH}$. Evaporation of the water afforded $1.06 \mathrm{~g}$ of a white solid, which was redissolved in $3 \mathrm{~mL} \mathrm{H}_{2} \mathrm{O}$ and cooled to o ${ }^{\circ} \mathrm{C}$. To that solution, $\mathrm{NaOH}$ ( $57 \mathrm{mg}, 1.43 \mathrm{mmol}, 1.5$ equiv.) was added, followed by THF ( $3 \mathrm{~mL}$ ). Finally, $\mathrm{Boc}_{2} \mathrm{O}(270 \mathrm{mg}, 1.24$ mmol, 1.3 equiv.) was also added and the mixture was allowed to warm to room temperature overnight. The reaction mixture was then washed twice with hexanes and the aqueous layer was acidified carefully with $1 \mathrm{M} \mathrm{HCl}$ until $\mathrm{pH}=1$. Then, the aqueous were extracted four times with EtOAc and the combined organic extracts were dried over $\mathrm{Na}_{2} \mathrm{SO}_{4}$, filtered and evaporated to afford $132 \mathrm{mg}$ of an amber oil. This material was dissolved in $\mathrm{MeOH} / \mathrm{EtOAc}$ and filtered through a silica plug to afford the title compound as a yellow oil (98 $\mathrm{mg}, 50 \%) .{ }^{1} \mathbf{H}$ NMR: $\left(400 \mathrm{MHz}, \mathrm{CD}_{3} \mathrm{OD}\right) \delta(\mathrm{ppm})=4.72-4.65(\mathrm{~m}, 1 \mathrm{H}), 4.57$ $(\mathrm{dd}, J=9.4,3.2 \mathrm{~Hz}, 1 \mathrm{H}), 4.39(\mathrm{~d}, 29.8 \mathrm{~Hz}, 2 \mathrm{H}), 1.46(\mathrm{~s}, 9 \mathrm{H}) .{ }^{19 F}$ NMR (376 MHz, CD 3 OD) $\delta$ (ppm) -230.19 (s, 1F).

tert-butyl (R)-(1-((3-(3-(N,N-dimethylsulfamoyl)phenyl)1-methyl-1H-indol-5-yl)amino)-3-fluoro-1-oxopropan-2yl)carbamate (53). A vial was charged with 47 (11 mg, 0.03 mmol, 1 equiv.) and 52 (14 mg, o.o7 mmol, 2 equiv.). Then, dry $\mathrm{CH}_{2} \mathrm{Cl}_{2}(250 \mu \mathrm{L})$ was added, followed by DIPEA $(35 \mu \mathrm{L}, 0.13$ mmol, 4 equiv.) and HATU ( $27 \mathrm{mg}, 0.07 \mathrm{mmol}, 2.1$ equiv.). The reaction mixture was then stirred at room temperature overnight. It was then washed with $1 \mathrm{M} \mathrm{HCl}$ and brine, dried over $\mathrm{Na}_{2} \mathrm{SO}_{4}$, filtered and evaporated to afford $32 \mathrm{mg}$ of a brown oil. Purification by flash column chromatography (EtOAc:hexanes 3:2) afforded the title compound as a yellow oil (12 mg, 69\%). 'H NMR: (400 MHz, CD 3 CN) $\delta(\mathrm{ppm})=8.65\left(\mathrm{~s},{ }_{1} \mathrm{H}\right), 8.23(\mathrm{~d}, J$ $=1.9 \mathrm{~Hz}, 1 \mathrm{H}), 8.02-7.97(\mathrm{~m}, 1 \mathrm{H}), 7.93(\mathrm{tt}, J=7.5,1.6 \mathrm{~Hz}, 1 \mathrm{H})$, $7.71-7.61(\mathrm{~m}, 1 \mathrm{H}), 7.65-7.56(\mathrm{~m}, 2 \mathrm{H}), 7.45-7.39(\mathrm{~m}, 1 \mathrm{H}), 7.39$ - $7.30(\mathrm{~m}, 1 \mathrm{H}), 4.85-4.59(\mathrm{~m}, 2 \mathrm{H}), 4.45(\mathrm{~d}, J=12.6 \mathrm{~Hz}, 1 \mathrm{H})$, $3.84\left(\mathrm{~d}, J=3.2 \mathrm{~Hz},{ }_{3} \mathrm{H}\right), 2.72(\mathrm{~s}, 6 \mathrm{H}), 1.45(\mathrm{~s}, 9 \mathrm{H}) .{ }^{19 F}$ NMR: $(376$ $\left.\mathrm{MHz}, \mathrm{CD}_{3} \mathrm{CN}\right) \delta(\mathrm{ppm})=-229.00(\mathrm{~s}, 1 \mathrm{~F})$.

(R)-2-amino-N-(3-(3-(N,N-dimethylsulfamoyl)phenyl)-1methyl-1H-indol-5-yl)-3-fluoropropanamide (21). To a solution of 53 (12 mg, o.02 mmol, 1 equiv.) in dry $\mathrm{CH}_{2} \mathrm{Cl}_{2}$ (200 $\mu \mathrm{L})$, TFA (200 $\mu \mathrm{L}, 2.6 \mathrm{mmol}, 131$ equiv.) was added. After $1 \mathrm{~h}$ at room temperature, the solvents were evaporated. Purification by RP-HPLC (using a SiliCycle SiliaChrom dtC18 semipreparative column $(5 \mu \mathrm{m}, 100 \AA$, $10 \times 250 \mathrm{~mm})$ with a flow rate of 5 $\mathrm{mL} / \mathrm{min}$ eluting with solvent (A: $0.1 \%$ TFA in water B: $0.1 \%$ TFA in MeCN) on a gradient of $(2 \rightarrow 100) \%)$ solvent B over 15 $\left.\min , t_{R}=6.60 \mathrm{~min}\right)$ afforded the TFA salt of 21 as a colourless solid (3 mg, 24\%). ' H NMR: (400 MHz, CD $3 \mathrm{CN}) \delta(\mathrm{ppm})=9.44$ $(\mathrm{s}, 1 \mathrm{H}), 8.24(\mathrm{~s}, 1 \mathrm{H}), 7.98(\mathrm{~s}, 1 \mathrm{H}), 7.91-7.89\left(\mathrm{~m},{ }_{1} \mathrm{H}\right), 7.66-7.58$ $(\mathrm{m}, 3 \mathrm{H}), 7.40(\mathrm{~d}, J=8.7 \mathrm{~Hz}, 1 \mathrm{H}), 7.32(\mathrm{~d}, J=8.8 \mathrm{~Hz}, 1 \mathrm{H}), 4.96$ (d, $J=46.5 \mathrm{~Hz}, 2 \mathrm{H}), 4.46(\mathrm{~s}, 1 \mathrm{H}), 3.82(\mathrm{~s}, 3 \mathrm{H}), 2.72(\mathrm{~s}, 6 \mathrm{H}) .{ }^{19 F}$ NMR: (376 MHz, CD $\mathrm{CN}) \delta(\mathrm{ppm})=-231.3(\mathrm{~s}, 1 \mathrm{~F})$. HRMS: (ESI) $\mathrm{m} / \mathrm{z}$ calculated for $\mathrm{C}_{20} \mathrm{H}_{23} \mathrm{FN}_{4} \mathrm{O}_{3} \mathrm{~S}[\mathrm{M}+\mathrm{H}]^{+} 419.1548$, found 419.1565 .

3-bromo-N,N-dimethylbenzamide (54). To a solution of $2 \mathrm{M}$ $\mathrm{Me}_{2} \mathrm{NH}$ in THF (11.4 mL, 23 mmol, 2 equiv.) in $25 \mathrm{~mL}$ dry $\mathrm{CH}_{2} \mathrm{Cl}_{2}$ at o ${ }^{\circ} \mathrm{C}, \mathrm{m}$-bromobenzoyl chloride $(1 \mathrm{~mL}, 7.6 \mathrm{mmol}, 1$ equiv.) was added dropwise. After the addition was complete, the reaction mixture was allowed to warm to room temperature overnight. After reaction was completed as observed by TLC, it was diluted with saturated $\mathrm{NaHCO}_{3}$. The layers were separated and the aqueous was extracted once more with $\mathrm{CH}_{2} \mathrm{Cl}_{2}$. The combined organic layers were dried over $\mathrm{Na}_{2} \mathrm{SO}_{4}$, filtered and evaporated to afford the title compound as a red oil (1.73 g, quantitative). ${ }^{1}$ H NMR: (50o $\left.\mathrm{MHz} \mathrm{CDCl}_{3}\right) \delta_{7.53}(\mathrm{t}$, $J=1.7 \mathrm{~Hz}, 1 \mathrm{H}), 7.51(\mathrm{ddd}, J=7.9,2.0,1.2 \mathrm{~Hz}, 1 \mathrm{H}), 7.31(\mathrm{dt}, J=7.7$, $1.3 \mathrm{~Hz}, 1 \mathrm{H}), 7.25-7.22(\mathrm{~m}, 1 \mathrm{H}), 3.01(\mathrm{~d}, J=64.7 \mathrm{~Hz}, 6 \mathrm{H})$.

N,N-dimethyl-3-(4,4,5,5-tetramethyl-1,3,2-dioxaborolan2-yl)benzamide (55). A vial was purged with $\mathrm{N}_{2}$ and charged with 54 (765 mg, 3.4 mmol, 1 equiv.), $B_{2}$ pin $_{2}$ (937 mg, 3.7 mmol, 1.1 equiv.), KOAc ( $1.65 \mathrm{~g}, 17 \mathrm{mmol}, 5$ equiv.), dioxane $(15 \mathrm{~mL})$ and $\mathrm{H}_{2} \mathrm{O}(2 \mathrm{~mL})$. The seal was removed temporarily and $\mathrm{Pd}(\mathrm{dppf}) \mathrm{Cl}_{2} . \mathrm{CH}_{2} \mathrm{Cl}_{2}$ (137 mg, $0.17 \mathrm{mmol}, 5 \mathrm{~mol} \%$ ) was added. The vial was then sealed again and microwaved for $2 \mathrm{~h}$ at 120 ${ }^{\circ} \mathrm{C}$. The reaction mixture was then diluted with saturated $\mathrm{Na}-$ $\mathrm{HCO}_{3}$ and brine and extracted with EtOAc (3 times). The combined organic layers were then washed with brine, dried over $\mathrm{Na}_{2} \mathrm{SO}_{4}$, filtered and evaporated to afford a brown oil (977mg). Purification by column chromatography (EtOAc:hexanes 3:1) afforded a yellow oil, which was a 1:1.7 mixture of $55: \mathrm{B}_{2}$ pin $_{2}$ ( $487 \mathrm{mg}, 21 \%)$. This material was used for the next step without further purification. ' $\mathbf{H}$ NMR: (40o $\mathrm{MHz} \mathrm{CDCl}_{3}$ ) $\delta 7.88-$ $7.78(\mathrm{~m}, 2 \mathrm{H}), 7.53-7.47(\mathrm{~m}, 1 \mathrm{H}), 7.39(\mathrm{td}, J=7.5,0.8 \mathrm{~Hz}, 1 \mathrm{H})$, $3.05(\mathrm{~d}, J=52.9 \mathrm{~Hz}, 6 \mathrm{H}), 1.34(\mathrm{~s}, 12 \mathrm{H})$.

tert-butyl 5-((tert-butoxycarbonyl)amino)-3-(3-(dimethylcarbamoyl)phenyl)-1H-indole-1-carboxylate (56). The title compound was prepared according to general procedure A using 7 (105 mg, $0.26 \mathrm{mmol}$, requiv.), 3-pinacolboronate arylbenzamide 55 mixture ( $77 \mathrm{mg}, 0.28 \mathrm{mmol}$, 1.1 equiv.) $\mathrm{K}_{2} \mathrm{CO}_{3}$ (106 mg, o.77 mmol, 3 equiv.), $\mathrm{Pd}(\mathrm{dppf}) \mathrm{Cl}_{2} \mathrm{CH}_{2} \mathrm{Cl}_{2}$ (21 mg, 0.03 mmol, o.1 equiv.), degassed dry THF (3.47 mL) and degassed $\mathrm{H}_{2} \mathrm{O}(1.13 \mathrm{~mL})$. Purification by column chromatography 
bioRxiv preprint doi: https://doi.org/10.1101/2020.11.17.387233; this version posted November 18, 2020. The copyright holder for this preprint (which was not certified by peer review) is the author/funder, who has granted bioRxiv a license to display the preprint in perpetuity. It is made available under aCC-BY-NC-ND 4.0 International license.

(EtOAc:hexanes 20:80 $\rightarrow$ 66:33) afforded the title compound as a brown oil $(81 \mathrm{mg}, 66 \%)$. ${ }^{1} \mathbf{H}$ NMR: $\left(400 \mathrm{MHz}, \mathrm{CDCl}_{3}\right) \delta$ $(\mathrm{ppm})=8.12(\mathrm{~d}, J=9.0 \mathrm{~Hz}, 1 \mathrm{H}), 7.86(\mathrm{~s}, 1 \mathrm{H}), 7.76-7.58(\mathrm{~m}$, $3 \mathrm{H}), 7.58-7.43(\mathrm{~m}, 1 \mathrm{H}), 7.44-7.35(\mathrm{~m}, 1 \mathrm{H}), 7.29(\mathrm{~d}, J=9.2 \mathrm{~Hz}$, $\left.{ }_{1 H}\right), 6.57(\mathrm{~s}, 1 \mathrm{H}), 3.08(\mathrm{~d}, J=26.3 \mathrm{~Hz}, 6 \mathrm{H}), 1.68(\mathrm{~s}, 9 \mathrm{H}), 1.52(\mathrm{~s}$, $9 \mathrm{H})$.

3-(5-(1,3-dioxoisoindolin-2-yl)-1-methyl-1H-indol-3-yl)$\mathbf{N}, \mathbf{N}$-dimethylbenzamide (57). The title compound was prepared according to the general procedures I, F and G using 56 (6o mg, $0.12 \mathrm{mmol})$, TFA (1.2 mL), phthalic anhydride (18 mg, $0.12 \mathrm{mmol})$, dry toluene (0.25 mL), $\mathrm{K}_{2} \mathrm{CO}_{3}(86 \mathrm{mg}, 0.62 \mathrm{mmol})$, MeI (o.024 mL, o.40 mmol) and dry DMF(o.63 mL). Purification by column chromatography (MeOH: $\mathrm{CH}_{2} \mathrm{Cl}_{2}$ 10:90) afforded ${ }_{5} 6$ as a pale yellow solid (42 mg, 85\%). ${ }^{1} \mathbf{H}$ NMR: (500 $\left.\mathrm{MHz}, \mathrm{CDCl}_{3}\right) \delta(\mathrm{ppm})=8.01(\mathrm{~s}, 2 \mathrm{H}), 7.96(\mathrm{~d}, J=3.1 \mathrm{~Hz}, 1 \mathrm{H})$, $7.95(\mathrm{~d}, J=3.1 \mathrm{~Hz}, 1 \mathrm{H}), 7.88(\mathrm{~d}, J=1.8 \mathrm{~Hz}, 1 \mathrm{H}), 7.79(\mathrm{~d}, J=3.1$ $\mathrm{Hz}, 1 \mathrm{H}), 7.78(\mathrm{~d}, J=3.1 \mathrm{~Hz}, 1 \mathrm{H}), 7.67\left(\mathrm{dt}, J=7.7,1.4 \mathrm{~Hz},{ }_{1} \mathrm{H}\right)$, $7.62(\mathrm{t}, J=1.4 \mathrm{~Hz}, 1 \mathrm{H}), 7.48(\mathrm{~d}, J=8.7 \mathrm{~Hz}, 1 \mathrm{H}), 7.43(\mathrm{t}, J=7.7$ $\left.\mathrm{Hz},{ }_{1} \mathrm{H}\right), 7.32(\mathrm{~s}, 1 \mathrm{H}), 7.29(\mathrm{dt}, J=7.4,1.3 \mathrm{~Hz}, 1 \mathrm{H}), 3.88\left(\mathrm{~s},{ }_{3} \mathrm{H}\right)$, $3.12(\mathrm{~s}, 3 \mathrm{H}), 3.02(\mathrm{~s}, 3 \mathrm{H})$.

3-(5-amino-1-methyl-1H-indol-3-yl)-N,N-dimethylbenzamide (58). The title compound was prepared according to general procedure $\mathrm{H}$ using 57 (42 $\mathrm{mg}$, o.10 $\mathrm{mmol}$ ), hydrazine hydrate $(7 \mathrm{mg}, 0.14 \mathrm{mmol})$ and $\mathrm{MeOH}(1.0 \mathrm{~mL})$. The crude product was directly used in the next step without further purification. 'H NMR: (500 $\left.\mathrm{MHz} \mathrm{CDCl}_{3}\right) \delta(\mathrm{ppm})=7.64(\mathrm{~d}, J=$ $1.5 \mathrm{~Hz}, 1 \mathrm{H}), 7.63(\mathrm{ddd}, J=4.4,1.8,1.4 \mathrm{~Hz}, 1 \mathrm{H}), 7.42(\mathrm{t}, J=7.6 \mathrm{~Hz}$, ${ }_{1 \mathrm{H}}, 7.26\left(\mathrm{~s},{ }_{1 \mathrm{H}}\right), 7.25\left(\mathrm{dt}, J=7.1,1.4 \mathrm{~Hz},{ }_{1 \mathrm{H}}\right), 7.23(\mathrm{~d}, J=2.2 \mathrm{~Hz}$, $1 \mathrm{H}), 7.16(\mathrm{~d}, J=7.6 \mathrm{~Hz}, 1 \mathrm{H}), 6.74(\mathrm{dd}, J=8.6,2.2 \mathrm{~Hz}, 1 \mathrm{H}), 3.76$ (s, $3 \mathrm{H}), 3.13$ (br s, $3 \mathrm{H}), 3.02$ (br s, $3 \mathrm{H})$.

(S)-3-(5-(2-aminopropanamido)-1-methyl-1H-indol-3-yl)N,N-dimethylbenzamide (22). The title compound was prepared according to general procedure $\mathrm{B}$ using the aryl-indole 58 (14 mg, o.o48 mmol), (tert-butoxycarbonyl)-L-alanine (11 $\mathrm{mg}, 0.057 \mathrm{mmol}$ ), PyBOP (30 mg, $0.24 \mathrm{mmol}$ ), DIPEA (o.041 $\mathrm{mL}, 0.057 \mathrm{mmol})$, and dry DMF (0.5 mL) followed by Bocdeprotection using TFA ( $0.5 \mathrm{~mL})$. Purification by RP-HPLC (using a SiliCycle SiliaChrom dtC18 semipreparative column (5 $\mu \mathrm{m}, 100 \AA$, $10 \times 250 \mathrm{~mm}$ ) with a flow rate of $5 \mathrm{~mL} / \mathrm{min}$ eluting with solvent (A: $0.1 \%$ TFA in water B: $0.1 \%$ TFA in MeCN) on a gradient of $(2 \rightarrow 100) \%$ ) solvent $B$ over $\left.15 \mathrm{~min}, \mathrm{t}_{\mathrm{R}}=5.95 \mathrm{~min}\right)$ of the crude deprotected product afforded the TFA salt of 22 as a colorless solid (7 mg, 40\%). ${ }^{1} \mathbf{H}$ NMR: (40o $\mathrm{MHz}, \mathrm{CD}_{3} \mathrm{CN}$ ) $\delta(\mathrm{ppm})=9.10(\mathrm{~s}, 1 \mathrm{H}), 8.22(\mathrm{~s}, 1 \mathrm{H}), 7.67(\mathrm{~d}, J=7.3,2 \mathrm{H}), 7.49(\mathrm{~s}$, $\left.{ }_{1 \mathrm{H}}\right), 7.46\left(\mathrm{t}, J=7.9 \mathrm{~Hz},{ }_{1 \mathrm{H}}\right), 7.38\left(\mathrm{~d}, J=8.8 \mathrm{~Hz},{ }_{1 \mathrm{H}}\right), 7.29(\mathrm{br} \mathrm{d}$, $J=8.8 \mathrm{~Hz}, 1 \mathrm{H}), 7.25(\mathrm{br} \mathrm{d}, J=7.6 \mathrm{~Hz}, 1 \mathrm{H}), 4.19(\mathrm{q}, J=6.4 \mathrm{~Hz}$, $1 \mathrm{H}), 3.80(\mathrm{~s}, 3 \mathrm{H}), 3.05($ br s, $3 \mathrm{H}), 2.99($ br s, $3 \mathrm{H}), 1.58(\mathrm{~d}, J=6.4$ $\left.\mathrm{Hz},{ }_{3} \mathrm{H}\right)$. ${ }^{13} \mathrm{C}$ NMR: (150 MHz, $\left.\mathrm{CD}_{3} \mathrm{CN}\right) \delta(\mathrm{ppm})=172.1,168.6$, $138.4,136.7,135.9,131.9,129.8,129.6,128.5,126.4,126.0,125.0$, 116.6, 116.0, 111.6, 111.3, 51.02, 39.89, 35.39, 33.50, 30.20, 17.57. HRMS: (ESI) $m / z$ calculated for $\mathrm{C}_{21} \mathrm{H}_{24} \mathrm{~N}_{4} \mathrm{O}_{2}[\mathrm{M}+\mathrm{H}]+{ }^{+} 365.4565$, found 365.1915 .

\section{ASSOCIATED CONTENT}

Supporting Information. The supporting information is available free of charge at http://pubs.acs.org.

\section{Corresponding Authors}

Robert Britton - Department of Chemistry, Simon Fraser University, 8888 University Drive, Burnaby, BC, Canada;

Phone: +1 778-782-4889; Email: rbritton@sfu.ca

Vijayaratnam Santhakumar - Structural Genomics Consortium, MaRS Centre, South Tower, 101 College Street, Suite 70o, University of Toronto, Toronto, ON, Canada Phone: +1 416978-7842; Email: santha.santhakumar@utoronto.ca

\section{ACKNOWLEDGMENT}

This work was supported by a Natural Sciences and Engineering Research Council (NSERC) Discovery Grant (RB 201906368), and NSERC CREATE Grant (432008-2013). The SGC is a registered charity (Number 1097737) that receives funds from AbbVie, Bayer Pharma AG, Boehringer Ingelheim, Canada Foundation for Innovation, Eshelman Institute for Innovation, Genome Canada through Ontario Genomics Institute [OGI-055], Innovative Medicines Initia-tive (EU/ EFPIA) [ULTRA-DD Grant No. 115766], Janssen, Merck KGaA, Darmstadt, Germany, MSD, Novartis Pharma AG, Ontario Ministry of Research, Innovation and Science (MRIS), Pfizer, São Paulo Research Foundation-FAPESP, Takeda, and Wellcome $\left[106169 / Z_{14} / Z\right]$.

\section{ABBREVIATIONS}

PRMT4, Protein Arginine Methyl Transferase 4, PRMT6, Protein Arginine Methyl Transferase 6, $\mathrm{IC}_{50}$, half maximal inhibitory concentration, Boc, tert-butyloxycarbonyl, NBS, N-bromosuccinimide, pyBOP, Benzotriazole-1-yl-oxy-tris-pyrrolidino-phosphonium hexafluorophosphate, $\mathrm{B}_{2} \mathrm{pin}_{2}$, Bis(pinacolato)diboron, $\mathrm{BAF}_{155}$, SMARCC1 gene protein

\section{REFERENCES}

(1) Schurter, B.T.; Koh, S.S.; Chen, D.; Bunick, G.J.; Harp, J.M.; Hanson, B.L.; Henschen-Edman, A.; Mackay, D.R.; Stallcup, M.R.; Aswad, D.W. Methylation of histone H-3 by coactivator-associated arginine methyltransferase-1 Biochem. 2001, 40, 5747-5756

(2) An, W.; Kim, J.; Roeder, R.G. Ordered cooperative functions of PRMT1, p3oo, and CARM1 in transcriptional activation by $\mathrm{P} 53$ Cell 2004, 117, 735-748

(3) Cheng, D.; Côté, J.; Shaaban, S.; Bedford, M.T. The arginine methyltransferase CARM1 regulates the coupling of transcription and mRNA processing Mol. Cell. 2007, 25, 7183

(4) El Messaoudi, S.; Fabbrizio, E.; Rodriguez, C.; Chuchana, P.; Fauquier, L.; Cheng, D.; Theillet, C.; Vandel, L.; Bedford, M.T.; Sardet, C. Coactivator-associated arginine methyltransferase-1 (CARM1) is a positive regulator of the cyclin E1 gene Proc. Natl. Acad. Sci. USA 2006, 103,1335113356

(5) Lee, Y.H.; Stallcup, M.R. Roles of protein arginine methylation in DNA damage signaling pathways: is CARM1 a lifeor-death decision point? Cell Cycle 2011, 10, 1343-1344

(6) Chen, S.L.; Loffler, K.A.; Chen, D.; Stallcup, M.R.; Muscat, G.E.O. The coactivator-associated arginine methyltransferase- 1 is necessary for muscle differentiation: CARM1 coactivates myocyte enhancer factor-2 J. Biol. Chem. 2oo2, 277, 4324-4333

(7) Wei, H.; Mundade, R.; Lange, K.C.; Lu, T. Protein arginine methylation of non-histone proteins and its role in diseases Cell Cycle 2014, 13, 32-41 

available under aCC-BY-NC-ND 4.0 International license.

(8) Fuhrmann, J.; Clancy, K.W.; Thompson, P.R. Chemical biology of protein arginine modifications in epigenetic regulation Chem. Rev. 2015, 115, 5413-5461

(9) Kim, Y.R.; Lee, B.K.; Park, R.Y.; Nguyen, N.T.X.; Bae, J.A.; Kwon, D.D.; Jung, C. Differential CARM1 expression in prostate and colorectal cancers BMC Cancer 2010, 10:197

(10) Hong, H.; Kao, C.; Jeng, M.H.; Eble, J.N.; Koch, M.O.; Gardner, T.A.; Zhang, S.; Li, L.; Pan, C.X.; Hu, Z.; MacLennan, G.T.; Cheng, L. Aberrant expression of CARM1, a transcriptional coactivator of androgen receptor, in the development of prostate carcinoma and androgen-independent status Cancer 2004, 101, 83-89

(11) Frietze, S.; Lupien, M.; Silver, P.A.; Brown, M. CARM1 regulates estrogen-stimulated breast cancer growth through up-regulation of E2F1 Cancer Res. 2008, 68, 301-306

(12) Habashy, H.O.; Rakha, E.A.; Ellis, I.O. Powe, D.G. The oestrogen receptor coactivator CARMı has an oncogenic effect and is associated with poor prognosis in breast cancer Breast Cancer Res. Treat. 2013, 140, 307-316

(13) Wang, L.; Zhao, Z.; Meyer, M.B.; Saha, S.; Yu, M.; Guo, A.; Wisinski, K.B.; Huang, W.; Cai, W.; Pike, J.W.; Yuan, M.; Ahlquist, P.; Xu, W. CARMi methylates chromatin remodeling factor $\mathrm{BAF} 155$ to enhance tumor progression and metastasis Cancer Cell. 2014, 25, 21-36

(14) Nakayama, K.; Szewczyk, M.M.; Dela Sena, C.; Wu, H.; Dong, A.; Zeng, H.; Li, F.; de Freitas, R.F.; Eram, M.S.; Schapira, M.;Baba, Y.; Kunitomo, M.; Cary, D.R.; Tawada, M.; Ohashi, A.; Imaeda, Y.; Saikatendu, K.H.; Grimshaw, C.E.; Vedadi, M.; Arrowsmith, C.H.; Barsyte-Lovejoy, D.; Kiba, A.; Tomita, D.; Brown, P.J. TP-o64, a potent and selective small molecule inhibitor of PRMT4 for multiple myeloma Oncotarget. 2018, 9, 18480-18493

(15) Drew, A.E.; Moradei, O.; Jacques, S.L.; Rioux, N.; BoriackSjodin, A.P.; Allain, C.; Porter Scott, M.; Jin, L.; Raimondi, A.; Handler, J.L.; Ott, H.M.; Kruger, R.G.; McCabe, M.T.; Sneeringer, C.; Riera, T.; Shapiro, G.; Waters, N.J.; Mitchell, L.H.; Duncan, K.W.; Moyer, M.P.; Copeland, R.A.; Smith, J.; Chesworth, R.; Ribich, S.A. Identification of a CARM1 inhibitor with potent in vitro and in vivo activity in preclinical models of multiple myeloma Sci. Rep. 2017, 7: 17993

(16) Shen, Y..; Szewczyk, M.M.; Eram, M.S.; Smil, D.; Kaniskan, H.Ü.; de Freitas, R.F.; Senisterra, G.; Li, F.; Schapira, M.; Brown, P.J.; Arrowsmith, C.H.; Barsyte-Lovejoy, D.; Liu, J.; Vedadi, M.; Jin, J. Discovery of a potent, selective, and cellactive dual inhibitor of protein arginine methyltransferase 4 and protein arginine methyltransferase $6 \mathrm{~J}$. Med. Chem. 2016, 59, 9124-9139

(17) Eram, M.S.; Shen, Y.; Szewczyk, M.; Wu, H.; Senisterra, G.; Li, F.; Butler, K.V.; Kaniskan, H.Ü.; Speed, B.A.; Dela Seña, C.; Dong, A.; Zeng, H.; Schapira, M.; Brown, P.J.; Arrowsmith, C.H.; Barsyte-Lovejoy, D.; Liu, J.; Vedadi, M.; Jin, J. A potent, selective, and cell-active inhibitor of human type I protein arginine methyltransferases ACS Chem. Biol. 2016, 11, 772-781

(18) Hu, H.; Qian, K.; Ho, M.C.; Zheng, Y.G. Small molecule inhibitors of protein arginine methyltransferases Expert Opin. Investig. Drugs 2016, 25, 335-358

(19) Sack, J.S.; Thieffine, S.; Bandiera, T.; Fasolini, M.; Duke, G.J.; Jayaraman, L.; Kish, K.F.; Klei, H.E.; Purandare, A.V.; Rosettani, P.; Troiani, S.; Xie, D.; Bertrand, J.A. Structural basis for CARM1 inhibition by indole and pyrazole inhibitors Biochem. J. 2011, 436, 331-339
(20) De Freitas, R.F.; Eram, M.S.; Smil, D.; Szewczyk, M.M.; Kennedy, S.; Brown, P.J.; Santhakumar, V.; BarsyteLovejoy, D.; Arrowsmith, C.H.; Vedadi, M.; Schapira, M. Discovery of a potent and selective coactivator associated arginine methyltransferase 1 (CARM1) inhibitor by virtual screening J. Med. Chem. 2016, 59, 6838-6847

(21) Cai, X.C.; Zhang, T.; Kim, E.J.; Jiang, M.; Wang, K.; Wang, J.; Chen, S.; Zhang, N.; Wu, H.; Li, F.; Dela Seña, C.; Zeng, H.; Vivcharuk, V.; Niu, X.; Zheng, W.; Lee, J.P.; Chen, Y.; Barsyte, D.; Szewczyk, M.; Hajian, T.; Ibañez, G.; Dong, A.; Dombrovski, L.; Zhang, Z.; Deng, H.; Min, J.; Arrowsmith, C.H.; Mazutis, L.; Shi, L.; Vedadi, M.; Brown, P.J.; Xiang, J.; Qin, L.X.; Xu, W.; Luo, M. A chemical probe of CARMı alters epigenetic plasticity against breast cancer cell invasion Elife 2019, 8:e47110

(22) Mitchell, L.H.; Drew, A.E.; Ribich, S.A.; Rioux, N.; Swinger, K.K.; Jacques, S.V.; Kaniskan, H.Ü.; Speed, B.A.; Dela Seña, C.; Dong, A.; Zeng, H.; Schapira, M.; Riera, T.; Porter-Scott, M.; Moyer, M.P.; Smith, J.J.; Chesworth, R.; Copeland, R.A. Aryl pyrazoles as potent inhibitors of arginine methyltransferases: identification of the first PRMT6 tool compound ACS Med. Chem. Lett. 2015, 6, 655-659

(23) Carpentier, C.; Godbout, R.; Otis, F.; Voyer, N. Synthesis and use of $N$-Fmoc-L-alanine Tet. Lett. 2015, 56, 244-1246

(24) De Koning, C.B.; Michael, J.P.; Rousseau, A.L.J. A versatile and convenient method for the synthesis of substituted benzo[a]carbazoles and pyrido[2,3]-carbazoles Chem. Soc. Perkin Trans. 1, 200o, o, 1705-1713

(25) Blough, B.E.; Landavazo, A.; Partilla, J.S.; Decker, A.M.; Page, K.M.; Baumann, M.H.; Rothman, R.B. Alpha-ethyltryptamines as dual dopamine-serotonin releasers Bioorg. Med. Chem. Lett. 2014, 24, 4754-4758

(26) Livendahl, M.; Jamroskovic, J.; Ivanova, S.; Demirel, P.; Sabouri, N.; Chorell, E. Design and synthesis of 2,2'-diindolylmethanes to selectively target certain G-quqdruplex DNA structures Chem - A Eur. J. 2016, 22, 13004-13009

(27) Taddei, M.; Cini, E.; Giannotti, L.; Giannini, G.; Battistuzzi, G.; Vignola, D.; Vesci, L.; Cabri, W. Lactam based 7-amino suberoylamide hydroxamic acids as potent HDAC inhibitors Bioorg. Med. Chem. Lett. 2014, 24, 61-64

(28) Valeur, E.; Bradley, M.; Amide bond formation: beyond the myth of coupling reagents Chem. Soc. Rev. 2oo9, 38, 6o6631 